\title{
Soft Information for Localization-of-Things
}

\author{
Andrea Conti, Senior Member, IEEE, Santiago Mazuelas, Senior Member, IEEE, \\ Stefania Bartoletti, Member, IEEE, William C. Lindsey, Life Fellow, IEEE, and Moe Z. Win, Fellow, IEEE
}

Soft information is much richer than single-value estimates.

Its exploitation opens the way to a new level of accuracy for the Localization-of-Things.

\begin{abstract}
Location awareness is vital for emerging Internetof-Things applications and opens a new era for Localization-ofThings. This paper first reviews classical localization techniques based on single-value metrics, such as range and angle estimates, and on fixed measurement models, such as Gaussian distributions with mean equal to the true value of the metric. Then, it presents a new localization approach based on soft information (SI) extracted from intra- and inter-node measurements, as well as from contextual data. In particular, efficient techniques for learning and fusing different kinds of SI are described. Case studies are presented for two scenarios in which sensing measurements are based on (i) noisy features and non-line-ofsight detector outputs, and (ii) IEEE 802.15.4a standard. Results show that SI-based localization is highly efficient, can significantly outperform classical techniques, and provides robustness to harsh propagation conditions.
\end{abstract}

Index Terms-Localization, wireless networks, learning, soft information, Internet-of-things, localization-of-things.

\section{INTRODUCTION}

Location awareness enables numerous wireless applications that rely on information associated with the positions of nodes such as anchors, agents, and targets in wireless networks [1]-[5]. These applications include autonomy [6]-[10], crowd sensing [11]-[19], smart environments [20]-[25], assets tracking [26]-[30], and the Internet-of-Things (IoT) [31]-[36]. The process of locating, tracking, and navigating any possible collaborative or non-collaborative nodes (devices, objects,

Manuscript submitted July 31st, 2017; revised April 14th, 2018; accepted Month ddth, 2018.

This research was supported, in part, by the Office of Naval Research under Grants N62909-18-1-2017 and N00014-16-1-2141, the Spanish Ministry of Economy and Competitiveness MINECO under Ramon y Cajal Grant RYC2016-19383 and BCAM Severo Ochoa excellence accreditation SEV-20170718, the European Union's H2020 research and innovation programme under the Marie Skłodowska-Curie Grant 703893, and the Copernicus Fellowship. (Corresponding author: Andrea Conti).

A. Conti is with the Department of Engineering and CNIT, University of Ferrara, 44122 Ferrara, Italy (e-mail: a. conti@ieee.org).

S. Mazuelas was with the Wireless Information and Network Sciences Laboratory, Massachusetts Institute of Technology, Cambridge, MA 02139, USA, and he is now with the BCAM-Basque Center for Applied Mathematics and IKERBASQUE-Basque Foundation for Science, 48009 Bilbao, Spain (email: smazuelas (bcamath. org).

S. Bartoletti is with the Department of Engineering and CNIT, University of Ferrara, 44122 Ferrara, Italy, and was with the Wireless Information and Network Sciences Laboratory, Massachusetts Institute of Technology, Cambridge, MA 02139, USA (e-mail: stefania.bartoletti@unife.it).

W. C. Lindsey is with the Ming Hsieh Department of Electrical Engineering, University of Southern California, Los Angeles, CA 90089, USA (e-mail: wclindsey@gmail.com).

M. Z. Win is with the Laboratory for Information and Decision Systems, Massachusetts Institute of Technology, Cambridge, MA 02139, USA (e-mail: moewin@mit.edu).

Digital Object Identifier 10.1109/JPROC.2019.XXXXXXX

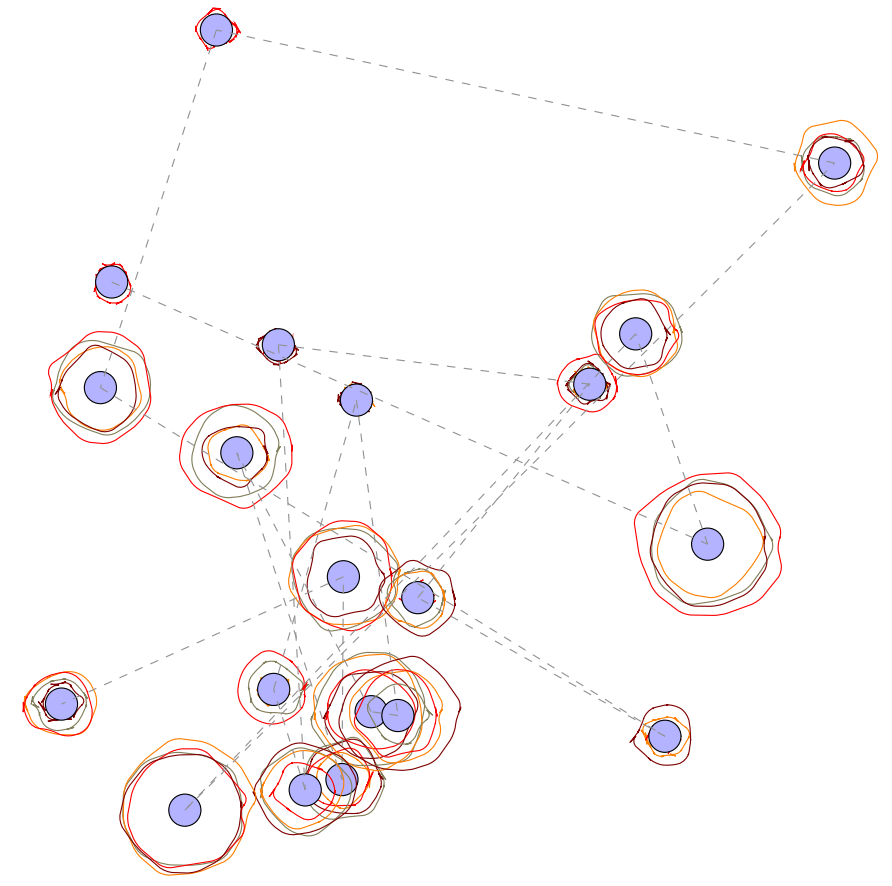

Fig. 1. Pictorial view of LoT relying on SI: blue circles represents nodes, dashed lines represents wireless connectivity between nodes, and red contours represent the SI values associated with node positions.

people, and vehicles) is referred to as Localization-of-Things $(L O T)$. The positional information of network nodes is encapsulated by soft information (SI), the ensemble of positional and environmental information respectively associated with measurements and contextual data. The SI can be extracted via sensing measurements (e.g., using radio, optical, and inertial signals) and contextual data (e.g., using digital map, dynamic model, and node profile). Fig. 1 provides a pictorial view of LoT relying on SI associated with each node. Accurate LoT depends on reliable acquisition and exploitation of SI, which can be challenging, especially in harsh wireless propagation environments. In particular, conventional approaches, based on fixed models, are often inadequate for describing SI as a function of the operating environment, signal features, and filtering techniques.

The demand for accurate localization is growing rapidly despite the difficulty in extracting positional information from received waveforms in most wireless environments. Research in localization and navigation has been carried out along four main strands: (a) fundamental limits [37]-[46]; (b) algorithm design [47]-[79]; (c) network operation [80]-[91]; and (d) network experimentation [92]-[96]. Conventional approaches 
to localization typically rely on the estimation of single values such as distances and angles from inter-node measurements, and accelerations and orientations from intra-node measurements. In particular, conventional approaches divide the localization process into two stages: (i) a single-value estimation stage in which distances, angles, accelerations, or other position-dependent quantities are estimated; and (ii) a localization stage in which prior knowledge and single-value estimates (SVEs) serve as inputs to a localization algorithm for position inference. For example, in conventional range-based localization and navigation, the positions of agents or targets are inferred from anchor positions and distance estimates [59][61]. Localization accuracy obtained by such methods depends heavily on the quality of the SVEs [96]-[114].

Typically, the accuracy and reliability of conventional localization techniques degrade in wireless environments due to biases in SVEs caused by multipath propagation and nonline-of-sight (NLOS) conditions. Performance limits on ranging were established in [115]-[127], while tractable models for range information were derived in [51]. To cope with wireless propagation impairments, conventional localization approaches focus on improving the estimation of single values [97]-[102], [128], [129]. Techniques to refine the SVE have been exploited by relying on models for SVEs errors (e.g., the bias induced by NLOS conditions) [98], [128], [129]. In addition, received waveforms containing reliable positional information can be selected based on features extracted from their samples [130]. Data fusion techniques can be used to improve the performance of SVE-based localization by considering the SVE of different features as independent [131]-[133] or by involving hybrid models that account for the relationship among different features [134]-[137].

To overcome the limitations of SVE-based localization, onestage techniques that employ measurements to directly obtain positions based on a prior model, namely direct positioning (DP), have been explored [138]-[146]. Recently, localization techniques that rely on a set of possible values rather than on single distance estimates (DEs), namely soft range information (SRI), have been developed [55]. In particular, algorithms to learn SRI based on unsupervised machine learning have been developed. To improve the localization performance it is essential to design localization networks that exploit SI, such as SRI or soft angle information (SAI), together with environmental information, such as contextual data. Contextual data for localization include digital maps, dynamic models, and user profiles [147]-[155].

The LoT scenarios offer the possibility to exploit different sensors that have limited resources for communication, computing, and memory [156]-[164]. In fact, unleashing the multi-sensor LoT requires fusion of data and measurements collected from heterogeneous sensors with limited resources for communication, computing, and memory [5], and design of efficient network operation strategies [80], [81], [85], [88], [90], [91], [165]-[167]. Multi-sensor LoT calls for distributed implementation of SI-based localization capable of fusing information from multimodal measurements and environmental knowledge. In addition, distributed localization algorithms require the communication of messages [47]-[49], [168], which may involve high dimensionality depending on the kind of SI. Therefore, it is vital to develop techniques for reducing the dimensionality of SI to make message-passing amenable for SI-based localization.

The fundamental questions related to SI for localization and navigation are:

- what gain can be reaped with SI-based methods compared to classical ones;

- how the SI can be learned from sensing measurements like received waveform samples;

- would SI be enriched by fusing information from different observables and information from the environment; and

- can SI-based algorithms for LoT be implemented efficiently and distributively?

The answers to these questions provide insights into the evolution of positional information at different stages of the localization process, which are essential for the design and analysis of localization systems. The goal of this paper is to establish the use of SI-based methods for LoT and quantify their performance gain with respect to classical ones. We advocate the exploitation of SI, which opens the way to a new level of accuracy for LoT.

This paper establishes SI-based methods for localization and navigation. In particular, it describes techniques for learning the SI and determines the benefits of fusing different types of positional information. It also demonstrates that SI is much richer than SVEs for localization and navigation. The key contributions of this paper include:

- introduction of SI-based techniques for LoT;

- methods for learning and fusing SI that is extracted from sensing measurements and contextual data; and

- quantification of the benefits provided by SI-based techniques compared to SVE-based and DP techniques.

Case studies are presented for two scenarios in which sensing measurements are based on (i) noisy features and non-line-ofsight detection, and (ii) IEEE 802.15.4a standard.

The remaining sections are organized as follows: Section II provides an overview of techniques for LoT. Section III defines SI for localization in terms of positional and environmental information. Section IV describes how SI can be exploited in localization and navigation. Section $\mathrm{V}$ provides performance benchmarks for SI-based localization. Section VI presents learning algorithms and data set reduction methods for SIbased localization. Section VII provides performance results for different case studies. Finally, Section VIII summarizes the paper.

Notations: $\mathbb{R}$ denotes the set of real numbers $\left(\mathbb{R}^{+}\right.$for non-negatives) and $\mathbb{R}^{M}$ its $M$-th Cartesian power. Random variables are displayed in sans-serif, upright fonts; their realizations in serif, italic fonts. Vectors are denoted by bold lowercase letters. For example, a random variable (RV) and its realization are denoted by $\mathrm{x}$ and $x$; a random vector and its realization are denoted by $\mathbf{x}$ and $\boldsymbol{x}$. The function $f_{\mathbf{x}}(\boldsymbol{x})$ and, for brevity when possible, $f(\boldsymbol{x})$ denote the probability density function (PDF) of a continuous RV $\mathbf{x} ; f_{\mathbf{x} \mid \mathbf{y}}(\boldsymbol{x} \mid \boldsymbol{y})$ and, for brevity when possible, $f(\boldsymbol{x} \mid \boldsymbol{y})$ denote the PDF of $\mathbf{x}$ conditional on $\mathbf{y}=\boldsymbol{y} ; \varphi(\boldsymbol{x} ; \boldsymbol{m}, \boldsymbol{\Sigma})$ denotes the PDF of a Gaussian random 
vector $\mathbf{x}$ with mean $\boldsymbol{m}$ and covariance matrix $\boldsymbol{\Sigma}$; operators $\mathbb{E}\{\cdot\}, \mathbb{V}\{\cdot\}$, and $\mathbb{P}\{\cdot\}$ denote, respectively, the expectation, variance, and probability of the argument, and $\mathbb{E}_{\times}\{\cdot\}$ denotes the expectation with respect to RV $x$. Sets are denoted by calligraphic fonts, e.g., $\mathcal{Y}$, and empty set is denoted by $\emptyset$. For a matrix $\boldsymbol{A}$ and a vector $\boldsymbol{a}$ the transpose is denoted by $\boldsymbol{A}^{\mathrm{T}}$ and $\boldsymbol{a}^{\mathrm{T}}$, respectively; $\operatorname{tr}\{\boldsymbol{A}\}$ denotes the trace of the matrix $\boldsymbol{A}$; and $\otimes$ denotes the Kronecker product of matrices. The norm of a vector $\boldsymbol{u}$ is denoted by $\|\boldsymbol{u}\|$. A positional feature vector, a measurement vector, ${ }^{1}$ and contextual data are respectively denoted by $\boldsymbol{\theta}, \boldsymbol{y}$, and $\boldsymbol{\mu}$. This paper considers both Bayesian and non-Bayesian formulations; in the former case, the relevant parameters are modeled as random.

\section{LOCALIZATION OF THINGS}

This section provides the problem setting, discusses the key aspects, and introduces techniques for LoT.

\section{A. Preliminaries}

A localization network is composed of $N_{\mathrm{a}}$ agents $^{2}$ with index set $\mathcal{N}_{\mathrm{a}}=\left\{1,2, \ldots, N_{\mathrm{a}}\right\}$ at unknown positions, and $N_{\mathrm{b}}$ anchors with index set $\mathcal{N}_{\mathrm{b}}=\left\{N_{\mathrm{a}}+1, N_{\mathrm{a}}+2, \ldots, N_{\mathrm{a}}+N_{\mathrm{b}}\right\}$ at known positions. Both the measurement collection and localization process are performed at discrete time instants, $t_{n}$, with index set $\mathcal{N}_{\mathrm{t}}=\left\{1,2, \ldots, N_{\mathrm{t}}\right\}$. The goal is to determine the positional state of agents at different time instants. The positional state of agent $i$ at time $t_{n}$, for $i \in \mathcal{N}_{\mathrm{a}}$ and $n \in \mathcal{N}_{\mathrm{t}}$, is denoted by $\boldsymbol{x}_{i}^{(n)} \in \mathbb{R}^{D}$ and includes the position $\boldsymbol{p}_{i}^{(n)}$ and other mobility parameters such as velocity $\boldsymbol{v}_{i}^{(n)}$, acceleration $\boldsymbol{a}_{i}^{(n)}$, orientation $\boldsymbol{\phi}_{i}^{(n)}$, and angular velocity $\boldsymbol{\omega}_{i}^{(n)}$. The concatenation of all agents' positional states and that of all agents' positions are denoted by $\boldsymbol{x}_{\mathcal{N}_{\mathrm{a}}}$ and $\boldsymbol{p}_{\mathcal{N}_{\mathrm{a}}}$, respectively. Localization techniques determine each position estimate $\hat{\boldsymbol{p}}_{i}$ based on a collection of measurements $\left\{\boldsymbol{y}_{i, j}\right\}_{j \in \mathcal{N}}$, where $\mathcal{N} \subseteq$ $\mathcal{N}_{\mathrm{a}} \cup \mathcal{N}_{\mathrm{b}}$ is the index set of nodes involved in measurements exchange with cardinality $N$, and on prior information such as previous positional states and environmental information.

Measurements are related to a feature vector $\boldsymbol{\theta}$ that is a function of node positional states. ${ }^{3}$ Therefore, the positional information can be extracted from measurements related to nodes $i$ and $j$ at time $t_{n}$, denoted by $\boldsymbol{y}_{i, j}^{(n)}$ for $i, j \in \mathcal{N}_{\mathrm{a}} \cup \mathcal{N}_{\mathrm{b}}$ and $n \in \mathcal{N}_{\mathrm{t}}$, where $i \neq j$ and $i=j$ correspond to interand intra-node measurements, respectively. An inter-node measurement between node $i$ and $j$ is related to positional states $\boldsymbol{x}_{i}$ and $\boldsymbol{x}_{j}$. Inter-node measurements are commonly obtained by radio measurement units and can include the entire set of received waveform samples or metrics such as received signal strength (RSS) [169]-[173], time-of-arrival (TOA) [174]-[178], time-difference-of-arrival (TDOA) [179][181], angle-of-arrival (AOA) [181]-[184], and Doppler shift [185]-[187]. An intra-node measurement of node $i$ is related

\footnotetext{
${ }^{1}$ In general, a measurement vector is a collection of measurements obtained by different types of sensors.

${ }^{2}$ Agents refer to any possible collaborative or non-collaborative nodes to be localized including devices, objects, people, and vehicles.

${ }^{3}$ For brevity, the dependence of $\boldsymbol{\theta}$ on node positional states will not explicitly be written in the following.
}

to the positional state $\boldsymbol{x}_{i}$. Intra-node measurements are commonly obtained by inertial measurement units (IMUs) and can include magnetic field intensity measurements, Doppler shift measurements, force measurements, and angular velocity measurements [151]-[153].

The environmental information $\boldsymbol{\mu}_{i}$ of agent $i$ can be used to enforce constraints on positional states. It is commonly composed of digital maps, dynamic models, and agent profiles [147]-[153]. A digital map for agent $i$ is related to its position $\boldsymbol{p}_{i}^{(n)}$ or consecutive positions $\boldsymbol{p}_{i}^{(n-1)}$ and $\boldsymbol{p}_{i}^{(n)}$; a dynamic model for agent $i$ is related to consecutive positional states $\boldsymbol{x}_{i}^{(n-1)}$ and $\boldsymbol{x}_{i}^{(n)}$; and an agent profile for agent $i$ is related to its positional state $\boldsymbol{x}_{i}^{(n)}$. In particular, digital maps can be used to discard positions that do not comply with the map (e.g., outside of a room or building, or not on a street) [147][149]; dynamic models can be used to express a positional state conditional to a previous state (e.g., moving within a certain speed interval and in a favorable direction) [188][191]; and agent profiles can be used to enforce relationships among positional state components (e.g., to enforce zero lateral and vertical velocities for vehicles when measured acceleration and angular velocity fall below given thresholds) [151]-[155].

The performance of localization systems is strongly affected by the quality of sensing measurements. For example, partial or complete blockage of line-of-sight (LOS) propagation conditions leads to positively biased range estimates for timebased ranging [92]. In fact, harsh wireless propagation conditions such as NLOS can result in highly biased estimates. Those impairments can be mitigated by detecting the wireless propagation conditions causing the bias. In such scenarios, a measurement vector is $\boldsymbol{y}=\left[\boldsymbol{z}^{\mathrm{T}}, \delta\right]^{\mathrm{T}}$ where $\boldsymbol{z}$ is a measurement vector related to a feature vector $\boldsymbol{\theta}$ and $\delta \in\{0,1\}$ is the NLOS detector outcome with 0 and 1 corresponding to detecting LOS and NLOS conditions, respectively. ${ }^{4}$ Detection errors are accounted for by means of posterior probabilities of error

$$
\begin{gathered}
\epsilon_{\mathrm{NLOS}} \triangleq \mathbb{P}\{\mathrm{NLOS} \mid \delta=0\}=\frac{p_{\mathrm{NLOS}}}{P_{0}} \mathbb{P}\{\delta=0 \mid \mathrm{NLOS}\} \\
\epsilon_{\mathrm{LOS}} \triangleq \mathbb{P}\{\operatorname{LOS} \mid \delta=1\}=\frac{1-p_{\mathrm{NLOS}}}{1-P_{0}} \mathbb{P}\{\delta=1 \mid \mathrm{LOS}\}
\end{gathered}
$$

where $p_{\mathrm{NLOS}}=\mathbb{P}\{\mathrm{NLOS}\}=1-\mathbb{P}\{\mathrm{LOS}\}$ is the probability of NLOS condition and $P_{0}=\mathbb{P}\{\delta=0\}=1-\mathbb{P}\{\delta=1\}$.

Classical approaches for identifying channel conditions are based on hypothesis testing, for example on binary hypothesis testing between LOS and NLOS conditions. Binary hypothesis testing can be extended to multiple hypothesis testing to identify one out of many (more than two) situations, for example, related to the number of obstacles (e.g., walls and furniture) that electromagnetic waves must traverse [92]. A key step in designing the decision rule is choosing the appropriate set of features extracted from received waveforms. Examples of features include delay spread, maximum amplitude, and kurtosis [98]-[101], [128], [129]. ${ }^{5}$

\footnotetext{
${ }^{4}$ For generic nodes, times, and features, the corresponding subscripts and superscripts will be omitted.

${ }^{5}$ In LOS conditions, the first path in the received signal is typically the strongest. LOS propagation conditions typically give rise to smaller delay spread and larger kurtosis compared to NLOS conditions [130].
} 


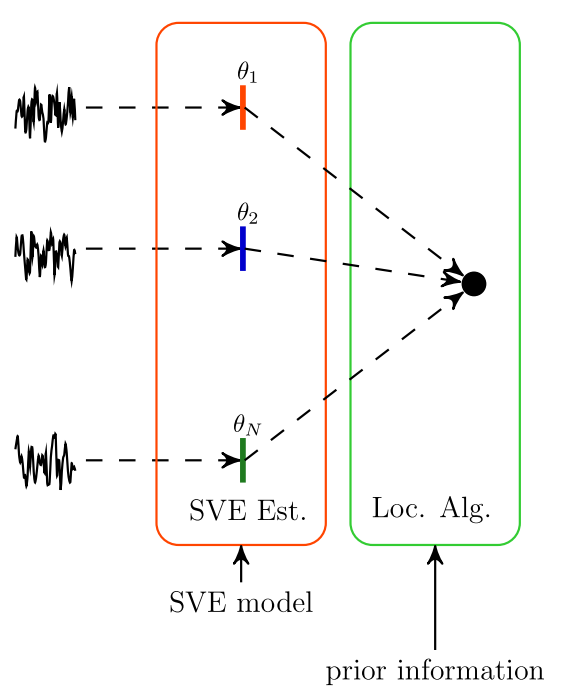

(a) Single Value Estimation

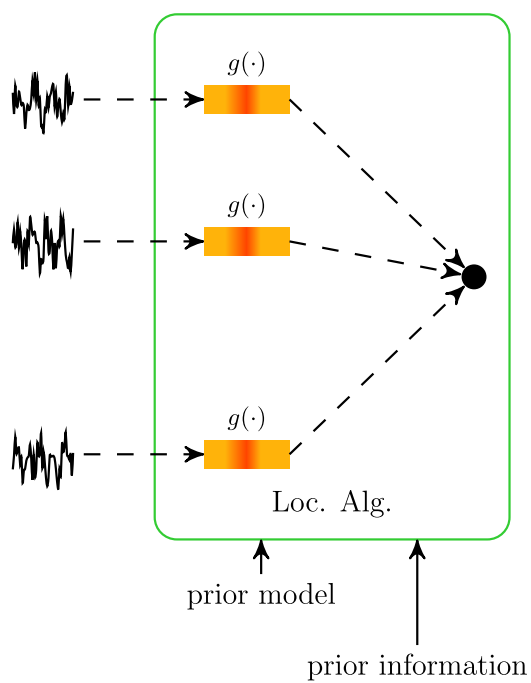

(b) Direct Positioning

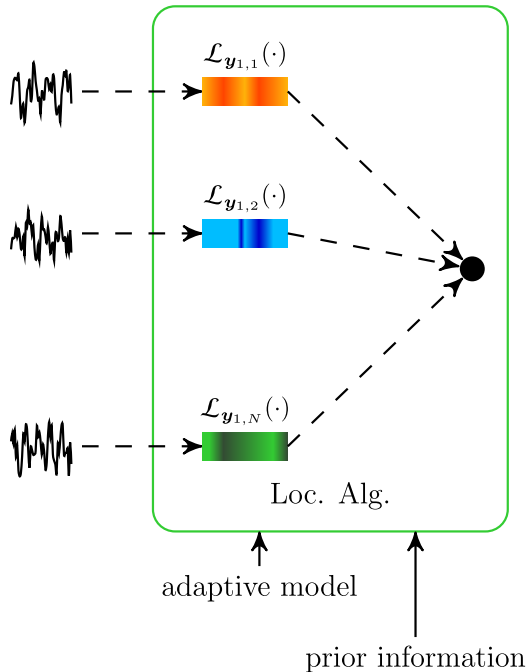

(c) Soft Information

Fig. 2. Sketches of localization techniques based on single values, direct positioning, and soft information.

Inference methods can be classified according to how agent positions are inferred (see Fig. 2) as described in the following.

\section{B. SVE-Based Techniques}

Classical techniques based on SVEs determine the position of agent $i \in \mathcal{N}_{\mathrm{a}}$ in two stages (see Fig. 2(a)) as described in the following.

(i) Estimation of single values: determine SVEs $\left\{\hat{\boldsymbol{\theta}}_{i, j}\right\}$ from inter- or intra-node measurements $\left\{\boldsymbol{y}_{i, j}\right\}_{j \in \mathcal{N}}$.

(ii) Positional inference: infer the positions $\boldsymbol{p}_{i}$ from SVEs $\left\{\hat{\boldsymbol{\theta}}_{i, j}\right\}_{j \in \mathcal{N}}$ using SVE-based algorithms (e.g., rangebased or angle-based algorithms).

The first stage processes each sensing measurement $\boldsymbol{y}_{i, j}$ to obtain a SVE $\hat{\boldsymbol{\theta}}_{i, j}$, such as DE for range-based localization [192]-[205], and angle estimate (AE) for direction-based localization [206]-[209]. The second stage infers the agent position $\boldsymbol{p}$ from the SVEs $\hat{\boldsymbol{\theta}}$, obtained in the first stage, using cooperative or non-cooperative algorithms.

An advantage of classical SVE-based techniques is that the first stage can be accomplished by independent procedures for each measurement $\boldsymbol{y}_{i, j}$. This can result in robust techniques since each measurement can be processed in a different manner (e.g., different procedures for processing measurements in LOS or NLOS conditions). Another advantage of classical SVE-based techniques is that the positional inference stage is simplified as its inputs are single values (e.g., multilateration localization algorithms). A disadvantage of classical SVEbased techniques is that the SVEs do not capture all the positional information contained in sensing measurements such as received waveform samples.

The localization accuracy of the two-stage approaches can be improved by:

(i) refining SVEs based on environmental information [92]; and

(ii) discarding SVEs from measurements that are unreliable for providing agent positional information [130]-[133].
Features extracted from sensing measurements can provide information useful in deciding whether a measurement is representative of the agent position or not (i.e., it contains information about agent position or it is due only to noise and background clutter) [130]. In cases where sensing measurements are not representative, they can be discarded and the corresponding SVEs are not used in the location inference. Other methods based on SVEs detect NLOS propagation conditions and then mitigate the errors on feature estimates when NLOS conditions are detected [97]-[101], [128], [129]. NLOS conditions typically introduce a bias $\beta$ on the expected value of the feature due to obstructed propagation. Therefore, the SVE $\hat{\theta}$ for a measurement $z$, based on the minimum-meansquare-error (MMSE) criterion, is given by [55]

$$
\hat{\theta}= \begin{cases}\left(1-\epsilon_{\mathrm{NLOS}}\right) z+\epsilon_{\mathrm{NLOS}}(z-\beta) & \text { for } \delta=0 \\ \epsilon_{\mathrm{LOS}} z+\left(1-\epsilon_{\mathrm{LOS}}\right)(z-\beta) & \text { for } \delta=1 .\end{cases}
$$

Note that, when the NLOS detector is highly reliable $\left(\epsilon_{\mathrm{NLOS}} \approx\right.$ $0, \epsilon_{\mathrm{LOS}} \approx 0$ ) the bias due to obstructed propagation is correctly subtracted to refine the SVE [92]. However, in the presence of NLOS detector error, SVEs are biased by $-\left(1-\epsilon_{\mathrm{LOS}}\right) \beta$ in LOS cases and by $\left(1-\epsilon_{\mathrm{NLOS}}\right) \beta$ in NLOS cases. For additive Gaussian noise with standard deviation $\sigma$ and $\epsilon_{\mathrm{NLOS}}=\epsilon_{\mathrm{LOS}}=\epsilon$, the mean-square-error (MSE) of the MMSE estimator is found to be

$$
\mathbb{E}\left\{|\hat{\theta}-\theta|^{2}\right\}=\epsilon(1-\epsilon) \beta^{2}+\sigma^{2} .
$$

This reduces to $\sigma^{2}$, which is the MSE in LOS propagation conditions, when the NLOS detector is totally reliable $(\epsilon=0)$.

\section{Direct Positioning Techniques}

DP techniques [138]-[146] estimate the position of agent $i$ by relying on the measurement model

$$
\mathbf{y}_{i, j}=g\left(\boldsymbol{\theta}_{i, j}\right)+\mathbf{n}
$$




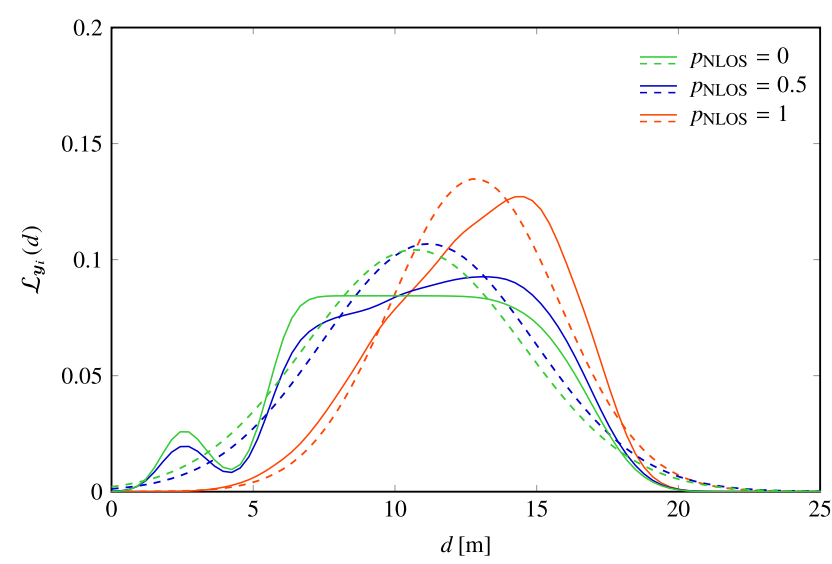

(a) IEEE 802.15.4 a indoor residential

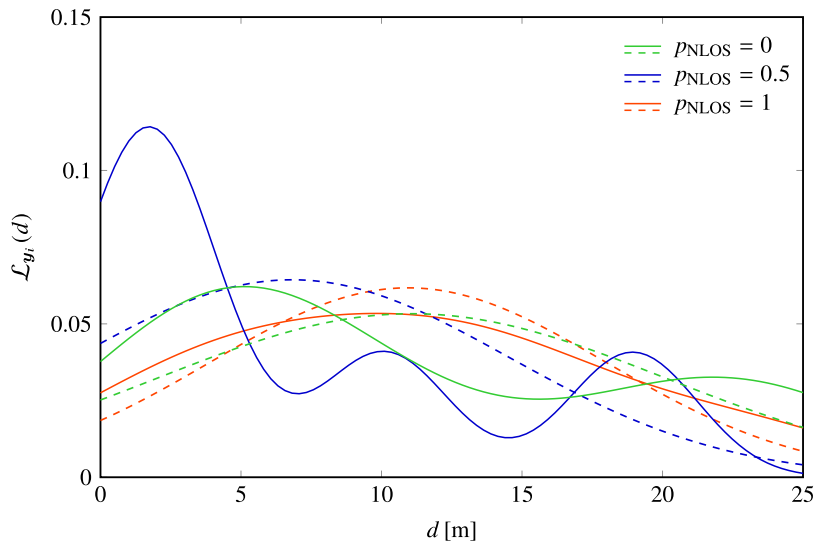

(b) IEEE 802.15.4 a outdoor

Fig. 3. Examples of distance likelihood functions in two IEEE 802.15 .4 a channels with NLOS probability $p_{\mathrm{NLOS}}=0$ (red), 0.5 (blue), and 1 (green). Empirical likelihoods (continuous lines) and Gaussian likelihoods with empirical mean and variance (dashed lines) are shown.

where the function $g(\cdot)$ is the same for all $j \in \mathcal{N}$ (see Fig. 2(b)), $\boldsymbol{\theta}_{i, j}$ depends on positions of nodes $i$ and $j$, and n represents additive white Gaussian noise. The position of node $i \in \mathcal{N}_{\mathrm{a}}$ is estimated as the maximum likelihood (ML) or least squares (LS) estimate based on (4). An advantage of DP techniques is that they can improve the localization accuracy with respect to SVE-based techniques since more information, intrinsically contained in sensing measurements, is used. Another advantage is that, when using a tractable $g(\cdot)$ together with independent, identically distributed Gaussian noise for each measurement, DP techniques can result in efficient implementations. A disadvantage of DP is that it is nonrobust in scenarios involving different propagation conditions (e.g., some measurements obtained in LOS and some other in NLOS). Another disadvantage is that it provides an inadequate performance when the knowledge of the function $g(\cdot)$ or the distribution of the noise $\mathbf{n}$ is not sufficiently accurate.

\section{SI-Based Techniques}

The SI-based techniques [55] directly use sensing measurements $\boldsymbol{y}_{i, j}$ from node $j \in \mathcal{N}$ to infer the position of node $i$ by relying on the SI $\mathcal{L}_{\boldsymbol{y}_{i, j}}\left(\boldsymbol{\theta}_{i, j}\right)$, which varies from measurement to measurement (see Fig. 2(c)). Such SI can encapsulate all the positional information in each sensing measurement. Then, the agent position $\boldsymbol{p}_{i}$ can be inferred from SI $\left\{\mathcal{L}_{\boldsymbol{y}_{i, j}}(\cdot)\right\}_{j \in \mathcal{N}}$.

An advantage of SI-based localization techniques is that the SI $\mathcal{L}_{\boldsymbol{y}}(\boldsymbol{\theta})$ can be obtained distributively by $N$ independent procedures tailored to the specific propagation conditions (e.g., either LOS or NLOS). Another advantage is that it can improve the localization accuracy by exploiting all the positional information in each sensing measurement. A disadvantage of SI-based localization techniques is that estimating the SI can be more complicated than estimating SVE.

To better understand the differences in models used for DP and SI-based techniques, Fig. 3 shows examples of distance likelihood function $\mathcal{L}_{\boldsymbol{y}}(d)$ for a fixed measurement $\boldsymbol{y}$, as a function of $d$, under different settings. The parameter $p_{\text {NLOS }}$ indicates the probability of NLOS propagation conditions. Fig. 3(a) and Fig. 3(b) show likelihood functions for a fixed sensing measurement $\boldsymbol{y}$ given by the maximum value of the ultrawide-band (UWB) waveforms in IEEE 802.15.4 a indoor residential and outdoor channels [210], respectively. The figures compare the empirical likelihood function and the Gaussian approximation (a model typically used in DP) using the empirical mean and variance. It can be seen from Figs. 3(a) and 3(b) that the Gaussian approximation is close to the empirical one in LOS conditions $\left(p_{\mathrm{NLOS}}=0\right)$, whereas it becomes less accurate as $p_{\mathrm{NLOS}}$ increases. In particular, the maxima of Gaussian approximations and empirical likelihoods occur at different distances in severe NLOS conditions and in equiprobable LOS/NLOS conditions. On the other hand, by attempting to learn the empirical likelihood, SI-based techniques can exploit richer information for better localization performance compared to SVE-based and DP techniques, especially in harsh propagation environments.

Remark 1: Note that, while DP considers the same form of likelihood function regardless of the propagation conditions for all sensing measurements, SI-based localization utilizes different forms of likelihood functions for measurements in different propagation conditions as depicted in Fig. 2. Observe that SIbased techniques reduce to DP or SVE-based techniques in specific cases. If $\mathbf{y}_{i, j}=\boldsymbol{\theta}_{i, j}+\mathbf{n}$ with $\mathbf{n}$ Gaussian noise, then the three approaches are equivalent. If the likelihood $\mathcal{L}_{\boldsymbol{y}_{i, j}}\left(\boldsymbol{\theta}_{i, j}\right)$ is proportional to a Gaussian PDF, then the approach based on SI is equivalent to that based on SVE. If the PDF $f\left(\boldsymbol{\theta}_{i, j} \mid \boldsymbol{y}_{i, j}\right)$ is proportional to $f_{\mathbf{n}}\left(\boldsymbol{y}_{i, j}-g\left(\boldsymbol{\theta}_{i, j}\right)\right)$, where $f_{\mathbf{n}}(\cdot)$ is the PDF of a zero-mean Gaussian random vector, then the approach based on SI is equivalent to DP.

\section{SOFT INFORMATION FOR LOCALIZATION}

SI is composed of soft feature information (SFI) and soft context information (SCI): SFI is the ensemble of positional information associated with measurements and SCI is the ensemble of environmental information associated with contextual data. SI-based localization infers agent positions by exploiting both SFI and SCI. 


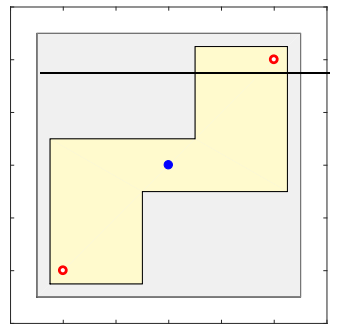

(a) Example scenario.

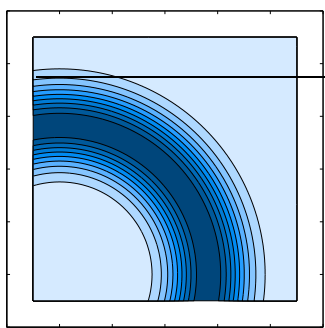

(b) SRI example.

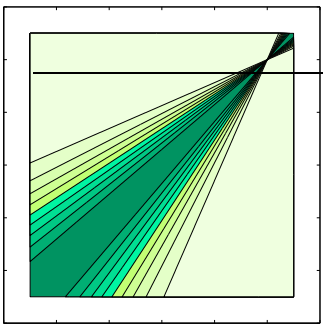

(c) SAI example.

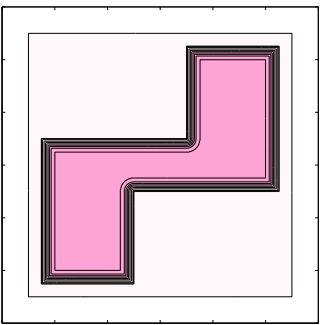

(d) SCI example.

Fig. 4. Examples of scenario and associated SI: the area is 120 meters by 120 meters; for the associated SI, darker colors refer to larger values.

\section{A. Soft Feature Information}

SFI for a measurement $\boldsymbol{y}$ is a function of the feature vector $\boldsymbol{\theta}$ given by ${ }^{6}$

$$
\begin{aligned}
& \mathcal{L}_{\boldsymbol{y}}(\boldsymbol{\theta}) \propto f_{\mathbf{y} \mid \theta}(\boldsymbol{y} \mid \boldsymbol{\theta}) \\
& \mathcal{L}_{\boldsymbol{y}}(\boldsymbol{\theta}) \propto f_{\mathbf{y}}(\boldsymbol{y} ; \boldsymbol{\theta})
\end{aligned}
$$

where (5a) and (5b) display the Bayesian and non-Bayesian formulation, respectively; in the latter case, SFI coincides with the likelihood function of feature vector $\boldsymbol{\theta}$. Different types of measurements give rise to different SFI. For instance, the SFI associated with range-related, angle-related, and velocityrelated measurements is respectively given by $\mathcal{L}_{\boldsymbol{y}}(d), \mathcal{L}_{\boldsymbol{y}}(\alpha)$, and $\mathcal{L}_{\boldsymbol{y}}(\boldsymbol{v})$.

Refer to the example scenario in Fig. 4(a) with two anchors (red annulus) and an agent (blue circle). The anchor in the bottom-left collects range-related measurements, from which the SRI of Fig. 4(b) is obtained. The anchor in the top-right collects angle-related measurements, from which the SAI of Fig. 4(c) is obtained. Thus, the SFI provides richer information than its SVE $\hat{\boldsymbol{\theta}}$ by quantifying the odds of different $\boldsymbol{\theta}$ values. The use of SFI enables soft-decision localization instead of classical hard-decision localization.

\section{B. Soft Context Information}

SCI is a function of the feature vector $\boldsymbol{\theta}$ provided by contextual data $\boldsymbol{\mu}$. Different types of contextual data, such as digital maps, dynamic models, and agent profiles, give rise to different kinds of SCI as described below.

The SCI provided by a map can be incorporated as a prior distribution of the position [147] (e.g., certain positions in the map are very unlikely) or as a conditional distribution of the position at time step $n$ given the position at time step $n-1$ (e.g., mobility in a corridor is more likely along the corridor then in a perpendicular direction). In the former case, the SCI is proportional to a prior distribution that depends on $\boldsymbol{\mu}$ as

$$
\Phi_{\boldsymbol{\mu}}(\boldsymbol{p}) \propto f_{\mathbf{p}}(\boldsymbol{p} ; \boldsymbol{\mu})
$$

while, in the latter case, it is proportional to a conditional PDF that depends on $\boldsymbol{\mu}$ as

$$
\Phi_{\boldsymbol{\mu}}\left(\boldsymbol{p}^{(n)}, \boldsymbol{p}^{(n-1)}\right) \propto f_{\mathbf{p}^{(n)} \mid \mathbf{p}^{(n-1)}}\left(\boldsymbol{p}^{(n)} \mid \boldsymbol{p}^{(n-1)} ; \boldsymbol{\mu}\right) .
$$

\footnotetext{
${ }^{6}$ The SFI and SCI are defined up to a proportionality constant, which is sufficient for SI-based localization.
}

SCI provided by a dynamic model can be incorporated as a conditional distribution of the positional state at time step $n$, given the positional state at time step $n-1$ (e.g., consecutive positions close to each other are highly likely for an agent with low speed; similar considerations apply to consecutive velocities for cars in a highway) [150]. Therefore, SCI associated with a dynamic model $\boldsymbol{\mu}$ is proportional to a conditional PDF depending on $\boldsymbol{\mu}$

$$
\Phi_{\boldsymbol{\mu}}\left(\boldsymbol{x}^{(n)}, \boldsymbol{x}^{(n-1)}\right) \propto f_{\mathbf{x}^{(n)} \mid \mathbf{x}^{(n-1)}}\left(\boldsymbol{x}^{(n)} \mid \boldsymbol{x}^{(n-1)} ; \boldsymbol{\mu}\right) .
$$

A widely used dynamic model is that based on a linearization of the positional state evolution via Taylor expansion and on Gaussian noise, leading to

$$
\Phi_{\boldsymbol{\mu}}\left(\boldsymbol{x}^{(n)}, \boldsymbol{x}^{(n-1)}\right) \propto \varphi\left(\boldsymbol{x}^{(n)} ; \boldsymbol{F} \boldsymbol{x}^{(n-1)}, \boldsymbol{\Sigma}_{d}\right)
$$

where $\boldsymbol{F}$ is known as the transition matrix and $\boldsymbol{\Sigma}_{d}$ is the covariance of the process noise, both depending on $\boldsymbol{\mu}$.

SCI provided by an agent profile can be incorporated as a distribution of several components in the positional state. For instance, if the agent is a pedestrian carrying the IMU on a foot, low values of acceleration and angular velocity correspond to high likelihood for the low values of velocity [153]. Therefore, the SCI provided by such agent profile $\boldsymbol{\mu}$ is proportional to a joint PDF of acceleration $\mathbf{a}$, angular velocity $\boldsymbol{\omega}$, and velocity $\mathbf{v}$ as

$$
\Phi_{\boldsymbol{\mu}}(\boldsymbol{a}, \boldsymbol{\omega}, \boldsymbol{v}) \propto f_{\mathbf{a}, \boldsymbol{\omega}, \mathbf{v}}(\boldsymbol{a}, \boldsymbol{\omega}, \boldsymbol{v} ; \boldsymbol{\mu}) .
$$

For example, if the agent is a car, misalignments of velocity vector and the direction of the car are highly unlikely. Therefore, SCI provided by such agent profile $\boldsymbol{\mu}$ is proportional to a PDF of the angle $\gamma$ between velocity $\mathbf{v}$ and heading $\mathbf{h}$ as

$$
\Phi_{\boldsymbol{\mu}}(\gamma) \propto f_{\gamma}(\gamma ; \boldsymbol{\mu})
$$

Refer to the example scenario in Fig. 4(a) with SCI given by the environment map depicted in Fig. 4(d). The SCI provides additional information on positional states, thus improving the performance of both soft-decision localization and classical hard-decision localization.

\section{Data Fusion Based on Soft Information}

The exploitation of SI for localization also enables the efficient fusion of sensing measurements and contextual data via multiplication of the corresponding SFI and SCI. 
Sensing measurements gathered with different modalities can be fused efficiently by multiplying their corresponding SFI as long as the different measurement vectors are conditionally independent given the positional features. Such conditional independence is generally satisfied as long as the measurement vectors are obtained from different sensors. In particular, for measurement set $\mathcal{Y}=\left\{\boldsymbol{y}^{(k)}\right\}_{k=1}^{K_{\mathrm{F}}}$ related to the feature set $\Theta=\left\{\boldsymbol{\theta}^{(k)}\right\}_{k=1}^{K_{\mathrm{F}}}$, with each measurement $\boldsymbol{y}^{(k)}$ related to feature $\boldsymbol{\theta}^{(k)}$ for $k=1,2, \ldots, K_{\mathrm{F}}$, SFI can be written as

$$
\mathcal{L}_{\mathcal{Y}}(\Theta)=\prod_{k=1}^{K_{\mathrm{F}}} \mathcal{L}_{\boldsymbol{y}^{(k)}}\left(\boldsymbol{\theta}^{(k)}\right) .
$$

\section{Soft Information in Harsh Propagation Environments}

SI can encapsulate all the positional information inherent in the sensing measurements obtained in harsh propagation environments even with errors on the detection of propagation conditions. Consider a measurement vector $\boldsymbol{y}=\left[\boldsymbol{z}^{\mathrm{T}}, \delta\right]^{\mathrm{T}}$ where $\boldsymbol{z}$ is a measurement vector related to a feature vector $\boldsymbol{\theta}$ and $\delta$ is the NLOS detector outcome as described in Section II-A. Assuming a constant reference prior for $\theta$ [211], the SFI of $\boldsymbol{y}$ is given by [55]

$$
\begin{aligned}
& \mathcal{L}_{\boldsymbol{y}}(\boldsymbol{\theta}) \propto \\
& \qquad \begin{cases}\left(1-\epsilon_{\mathrm{NLOS}}\right) \mathcal{L}_{\boldsymbol{z}_{\mathrm{LOS}}}(\boldsymbol{\theta})+\epsilon_{\mathrm{NLOS}} \mathcal{L}_{\boldsymbol{z}_{\mathrm{NLOS}}}(\boldsymbol{\theta}) & \text { for } \delta=0 \\
\epsilon_{\mathrm{LOS}} \mathcal{L}_{\boldsymbol{z}_{\mathrm{LOS}}}(\boldsymbol{\theta})+\left(1-\epsilon_{\mathrm{LOS}}\right) \mathcal{L}_{\boldsymbol{z}_{\mathrm{NLOS}}}(\boldsymbol{\theta}) & \text { for } \delta=1\end{cases}
\end{aligned}
$$

where $\mathcal{L}_{\boldsymbol{z}_{\mathrm{LOS}}}(\cdot)$ and $\mathcal{L}_{\boldsymbol{z}_{\mathrm{NLOS}}}(\cdot)$ denote the SFI for measurements collected in LOS and NLOS conditions, respectively.

For instance, consider a one-dimensional measurement model

$$
\mathrm{z}=\theta+\mathrm{n}
$$

where $\mathrm{n}$ represents the Gaussian noise with PDF

$$
f_{\mathrm{n}}(n)= \begin{cases}\varphi\left(n ; 0, \sigma_{\mathrm{LOS}}^{2}\right) & \text { for LOS cases } \\ \varphi\left(n ; \beta, \sigma_{\mathrm{NLOS}}^{2}\right) & \text { for NLOS cases }\end{cases}
$$

in which $\beta$ denotes the bias due to NLOS propagation. In such a case,

$$
\begin{aligned}
\mathcal{L}_{z_{\mathrm{LOS}}}(\theta) & =\varphi\left(\theta ; z, \sigma_{\mathrm{LOS}}^{2}\right) \\
\mathcal{L}_{z_{\mathrm{NLOS}}}(\theta) & =\varphi\left(\theta ; z-\beta, \sigma_{\mathrm{NLOS}}^{2}\right) .
\end{aligned}
$$

When the detector is highly reliable $\left(\epsilon_{\mathrm{NLOS}} \approx 0, \epsilon_{\mathrm{LOS}} \approx 0\right)$, $\mathrm{SFI}$ is concentrated around the true feature, i.e., $\mathcal{L}_{z_{\mathrm{LOS}}}(\theta)$ for LOS and $\mathcal{L}_{z_{\mathrm{NLOS}}}(\theta)$ for NLOS propagation conditions. Moreover, SI-based techniques are more robust to detector errors than classical techniques, as the SFI in (13) accounts for the error probability of the detector and considers both the true and biased features.

\section{SI-BASEd LOCALIZATION}

An SI-based localization system operates according to the following steps:

(i) acquisition of feature-related measurements and contextual data; (ii) characterization of the SFI and SCI provided by each measurement and contextual data; and

(iii) position inference by exploiting SFI and SCI.

To illustrate the benefits of SI for localization, we now describe how SFI and SCI can be utilized to infer the positions of the agents $\left\{\boldsymbol{p}_{i}\right\}_{i \in \mathcal{N}_{\mathrm{a}}}$ from measurements $\boldsymbol{y}$ and contextual data $\boldsymbol{\mu}{ }^{7}$ Recall that the feature vector $\boldsymbol{\theta}$ inherent in $\boldsymbol{y}$ is related to the node positions $\boldsymbol{p}$. In the following, we describe:

- localization without cooperation, where sensing measurements and contextual data are related only to one agent at a single time instant;

- network localization with spatial cooperation among agents, where sensing measurements and contextual data are related to neighboring agents at a single time instant;

- navigation with temporal cooperation, where sensing measurements and contextual data are related only to one agent at consecutive time instants; and

- network navigation with spatiotemporal cooperation, where sensing measurements and contextual data are related to neighboring agents at consecutive time instants.

\section{A. SI-Based Localization without Cooperation}

In non-cooperative localization systems, the positions of the agents are inferred based on measurements with respect to the anchors and contextual data. By modeling the positions of agents as unknown parameters, the ML estimate of the position of agent $i \in \mathcal{N}_{\mathrm{a}}$ is [55]

$$
\begin{aligned}
\hat{\boldsymbol{p}}_{i} & =\arg \max _{\boldsymbol{p}_{i}} f\left(\left\{\boldsymbol{y}_{i, j}\right\}_{j \in \mathcal{N}_{\mathrm{b}}} \mid \boldsymbol{p}_{i}\right) \\
& =\arg \max _{\boldsymbol{p}_{i}} \prod_{j \in \mathcal{N}_{\mathrm{b}}} \mathcal{L}_{\boldsymbol{y}_{i, j}}\left(\boldsymbol{\theta}_{i, j}\right) .
\end{aligned}
$$

If all the SFI in (17) are Gaussian with mean $\boldsymbol{\theta}_{i, j}$, then the ML estimator leads to the LS estimator and to the weighted least squares (WLS) estimator, respectively, for cases with same variance and different variances for $j \in \mathcal{N}_{\mathrm{b}}$.

By modeling the positions of agents as RVs, contextual data can be incorporated directly. The position of agent $i \in \mathcal{N}_{\mathrm{a}}$ can be inferred from the posterior distribution. In particular, MMSE and the maximum a posteriori (MAP) estimates are given by the mean and mode of the posterior distribution respectively as [55]

$$
\begin{aligned}
& \hat{\boldsymbol{p}}_{i}=\int \boldsymbol{p}_{i} f\left(\boldsymbol{p}_{i} \mid\left\{\boldsymbol{y}_{i, j}\right\}_{j \in \mathcal{N}_{\mathrm{b}}} ; \boldsymbol{\mu}_{i}\right) d \boldsymbol{p}_{i} \\
& \hat{\boldsymbol{p}}_{i}=\arg \max _{\boldsymbol{p}_{i}} f\left(\boldsymbol{p}_{i} \mid\left\{\boldsymbol{y}_{i, j}\right\}_{j \in \mathcal{N}_{\mathrm{b}}} ; \boldsymbol{\mu}_{i}\right)
\end{aligned}
$$

where for the posterior distribution

$$
f\left(\boldsymbol{p}_{i} \mid\left\{\boldsymbol{y}_{i, j}\right\}_{j \in \mathcal{N}_{\mathrm{b}}} ; \boldsymbol{\mu}_{i}\right) \propto \Phi_{\boldsymbol{\mu}_{i}}\left(\boldsymbol{p}_{i}\right) \prod_{j \in \mathcal{N}_{\mathrm{b}}} \mathcal{L}_{\boldsymbol{y}_{i, j}}\left(\boldsymbol{\theta}_{i, j}\right) .
$$

The contextual data $\boldsymbol{\mu}_{i}$ may depend on the previously estimated position of node $i$ (e.g., a map-aided 3D localization in which the map for node $i$ depends on whether the previously

\footnotetext{
${ }^{7}$ For notational convenience, consider that, for each pair of nodes $i$ and $j$, there is a measurement vector $\boldsymbol{y}_{i, j}$ or a contextual data vector $\boldsymbol{\mu}_{i}$ available. The expressions with unavailable measurements or data for some node pairs can be obtained by removing the terms corresponding to those pairs.
} 
estimated position for node $i$ was on a certain floor). Note that the MAP estimator in (18b) coincides with the ML estimator when contextual data are not available (i.e., $\Phi_{\boldsymbol{\mu}_{i}}\left(\boldsymbol{p}_{i}\right)$ constant with respect to $\boldsymbol{p}_{i}$ ).

\section{B. SI-Based Localization with Spatial Cooperation}

In network localization systems [1], the positions of the agents are inferred based on measurements with respect to neighboring agents, in addition to those with respect to the anchors, and to contextual data. By modeling the positions of agents as unknown parameters, the ML estimate of the positions of all the agents is [55]

$$
\begin{aligned}
\hat{\boldsymbol{p}}_{\mathcal{N}_{\mathrm{a}}} & =\underset{\boldsymbol{p}_{\mathcal{N}_{\mathrm{a}}}}{\arg \max } f\left(\left\{\boldsymbol{y}_{i, j}\right\}_{\substack{i \in \mathcal{N}_{\mathrm{a}} \\
j \in \mathcal{N}_{\mathrm{a}} \cup \mathcal{N}_{\mathrm{b}}}} \mid \boldsymbol{p}_{\mathcal{N}_{\mathrm{a}}}\right) \\
& =\underset{\boldsymbol{p}_{\mathcal{N}_{\mathrm{a}}}}{\arg \max } \prod_{\substack{i \in \mathcal{N}_{\mathrm{a}} \\
j \in \mathcal{N}_{\mathrm{a}} \cup \mathcal{N}_{\mathrm{b}}}} \mathcal{L}_{\boldsymbol{y}_{i, j}}\left(\boldsymbol{\theta}_{i, j}\right) .
\end{aligned}
$$

If all the SFI used in (20) are Gaussian with mean $\boldsymbol{\theta}_{i, j}$, then the ML estimate leads to LS or WLS estimates as in the noncooperative case.

By modeling the positions of agents as RVs, contextual data can be incorporated directly. The MMSE and the MAP estimates of all agent positions can be obtained analogously to (18) using the posterior distribution

$$
\begin{aligned}
f\left(\boldsymbol{p}_{\mathcal{N}_{\mathrm{a}}} \mid\left\{\boldsymbol{y}_{i, j}\right\}_{\substack{i \in \mathcal{N}_{\mathrm{a}} \\
j \in \mathcal{N}_{\mathrm{a} \cup \mathcal{N}_{\mathrm{b}}}}} ; \boldsymbol{\mu}_{\mathcal{N}_{\mathrm{a}}}\right) \\
\propto \prod_{i \in \mathcal{N}_{\mathrm{a}}}\left[\Phi_{\boldsymbol{\mu}_{i}}\left(\boldsymbol{p}_{i}\right) \prod_{j \in \mathcal{N}_{\mathrm{a}} \cup \mathcal{N}_{\mathrm{b}}} \mathcal{L}_{\boldsymbol{y}_{i, j}}\left(\boldsymbol{\theta}_{i, j}\right)\right] .
\end{aligned}
$$

Note that the MAP estimate coincides with ML estimate when contextual data are not available.

\section{SI-Based Navigation with Temporal Cooperation}

Navigation systems [1] infer the positions of the agents at different time instants based on inter-node measurements from the anchors, intra-node measurements, and contextual data. When positional states and measurements can be described by a hidden Markov model (HMM) over time steps from 1 to $n+1$, the posterior distribution of positional state for each agent can be obtained sequentially [53]. In particular, $f\left(\boldsymbol{x}_{i}^{(n+1)} \mid \mathcal{Y}_{i}^{(1: n+1)} ; \boldsymbol{\mu}_{i}\right)$ can be obtained by performing a prediction step using a dynamic model

$$
\begin{aligned}
f\left(\boldsymbol{x}_{i}^{(n+1)} \mid \mathcal{Y}_{i}^{(1: n)} ; \boldsymbol{\mu}_{i}\right) \propto \\
\quad \int \Phi_{\boldsymbol{\mu}_{i}}\left(\boldsymbol{x}_{i}^{(n+1)}, \boldsymbol{x}_{i}^{(n)}\right) f\left(\boldsymbol{x}_{i}^{(n)} \mid \mathcal{Y}_{i}^{(1: n)} ; \boldsymbol{\mu}_{i}\right) d \boldsymbol{x}_{i}^{(n)}
\end{aligned}
$$

followed by an update step using a new measurement

$$
\begin{aligned}
f\left(\boldsymbol{x}_{i}^{(n+1)} \mid \mathcal{Y}_{i}^{(1: n+1)} ; \boldsymbol{\mu}_{i}\right) \propto & \\
& \prod_{j \in \mathcal{N}_{\mathrm{b}} \cup\{i\}} \mathcal{L}_{\boldsymbol{y}_{i, j}^{(n+1)}}\left(\boldsymbol{\theta}_{i, j}^{(n+1)}\right) f\left(\boldsymbol{x}_{i}^{(n+1)} \mid \mathcal{Y}_{i}^{(1: n)} ; \boldsymbol{\mu}_{i}\right)
\end{aligned}
$$

where $\mathcal{Y}_{i}^{(1: k)}=\left\{\boldsymbol{y}_{i, j}^{(1: k)}\right\}_{j \in \mathcal{N}_{\mathrm{b}} \cup\{i\}} \cdot{ }^{8}$

$$
{ }^{8} \text { Notation } \boldsymbol{y}_{i, j}^{(1: k)} \text { denotes the set }\left\{\boldsymbol{y}_{i, j}^{(h)}\right\}_{h=1}^{k} \text {. }
$$

If both the SFI and SCI are Gaussian and linear with respect to positional states, then the updates in (23) can be performed in a closed form like those in Kalman filters (KFs) [212][216]. Otherwise, the implementation of (23) has to resort to approximations accounting for the complexity vs. accuracy tradeoff. Examples of such approximations are those used in extended Kalman filters (EKFs) [217], unscented Kalman filters (UKFs) [218], and belief condensation filters (BCFs) [53].

\section{SI-Based Navigation with Spatiotemporal Cooperation}

Network navigation systems [1] infer the positions of the agents at different time instants based on inter-node measurements with respect to both anchors and neighboring agents, intra-node measurements, and contextual data. When positional states and measurements can be described by a HMM over time steps from 1 to $n+1$, the joint posterior distribution of positional states can be obtained sequentially [40]. In particular, $f\left(\boldsymbol{x}_{\mathcal{N}_{\mathrm{a}}}^{(n+1)} \mid \mathcal{Y}^{(1: n+1)} ; \boldsymbol{\mu}_{\mathcal{N}_{\mathrm{a}}}\right)$ can be obtained by performing a prediction step using a dynamic model

$$
\begin{aligned}
& f\left(\boldsymbol{x}_{\mathcal{N}_{\mathrm{a}}}^{(n+1)} \mid \mathcal{Y}^{(1: n)} ; \boldsymbol{\mu}_{\mathcal{N}_{\mathrm{a}}}\right) \propto \\
& \quad \int \Phi_{\boldsymbol{\mu}_{\mathcal{N}_{\mathrm{a}}}}\left(\boldsymbol{x}_{\mathcal{N}_{\mathrm{a}}}^{(n+1)}, \boldsymbol{x}_{\mathcal{N}_{\mathrm{a}}}^{(n)}\right) f\left(\boldsymbol{x}_{\mathcal{N}_{\mathrm{a}}}^{(n)} \mid \mathcal{Y}^{(1: n)} ; \boldsymbol{\mu}_{\mathcal{N}_{\mathrm{a}}}\right) d \boldsymbol{x}_{\mathcal{N}_{\mathrm{a}}}^{(n)}
\end{aligned}
$$

followed by an update step using a new measurement

$$
\begin{aligned}
f\left(\boldsymbol{x}_{\mathcal{N}_{\mathrm{a}}}^{(n+1)} \mid \mathcal{Y}^{(1: n+1)} ; \boldsymbol{\mu}_{\mathcal{N}_{\mathrm{a}}}\right) \propto \\
\quad \prod_{\substack{i \in \mathcal{N}_{\mathrm{a}} \\
j \in \mathcal{N}_{\mathrm{a}} \cup \mathcal{N}_{\mathrm{b}}}} \mathcal{L}_{\boldsymbol{y}_{i, j}^{(n+1)}}\left(\boldsymbol{\theta}_{i, j}^{(n+1)}\right) f\left(\boldsymbol{x}_{\mathcal{N}_{\mathrm{a}}}^{(n+1)} \mid \mathcal{Y}^{(1: n)} ; \boldsymbol{\mu}_{\mathcal{N}_{\mathrm{a}}}\right)
\end{aligned}
$$

where $\mathcal{Y}^{(1: k)}=\left\{\boldsymbol{y}_{i, j}^{(1: k)}\right\}_{i \in \mathcal{N}_{\mathrm{a}}, j \in \mathcal{N}_{\mathrm{a}} \cup \mathcal{N}_{\mathrm{b}}}$.

If the movement of each agent is independent of any other agent's movement, then

$$
\Phi_{\boldsymbol{\mu}_{\mathcal{N}_{\mathrm{a}}}}\left(\boldsymbol{x}_{\mathcal{N}_{\mathrm{a}}}^{(n+1)}, \boldsymbol{x}_{\mathcal{N}_{\mathrm{a}}}^{(n)}\right)=\prod_{i \in \mathcal{N}_{\mathrm{a}}} \Phi_{\boldsymbol{\mu}_{i}}\left(\boldsymbol{x}_{i}^{(n+1)}, \boldsymbol{x}_{i}^{(n)}\right) .
$$

If both the SFI and SCI are Gaussian and linear with respect to positional states, then the updates in (25) can be performed in closed form as those in KFs [212]-[216]. Otherwise, the implementation of (25) has to resort to approximations accounting for the complexity vs. accuracy trade-off. Examples of such approximations are those used in EKFs [217], UKFs [218], and BCFs [53].

\section{E. SI-Based vs. SVE-Based Localization}

SI-based localization is a new approach that exploits richer information than classical SVE-based localization. Consider, for instance, range and angle inter-node measurements: the SI-based localization relies on SRI and SAI, whereas SVE based localization relies on range and angle estimates. Refer to the examples of SFI and SCI in Fig. 4. Fusion of all available SFI and SCI provides enhanced SI (red contoured areas in Fig. 5). In particular, Fig. 5(a) shows the fusion of the SFI corresponding to the SRI in Fig. 4(b) and to the SAI in Fig. 4(c). Fig. 5(b) shows the enhanced SI obtained by fusion 


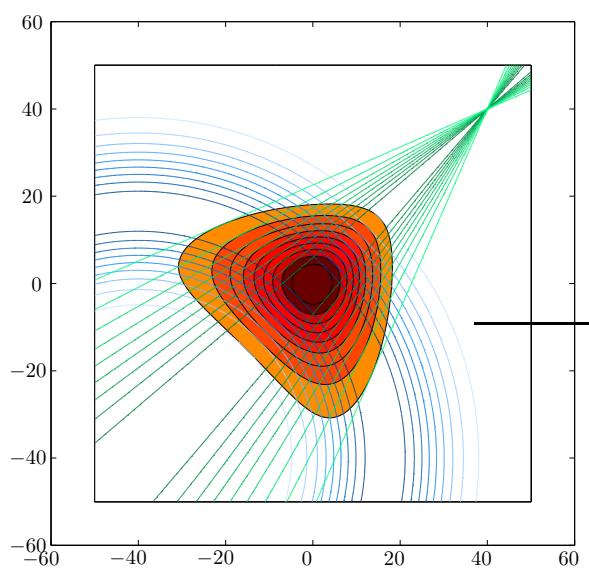

(a) Fusion of SRI and SAI.

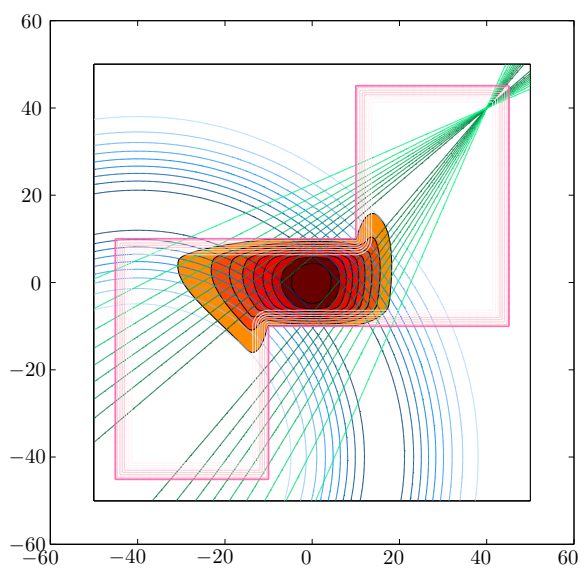

(b) Fusion of SRI, SAI, and SCI.

Fig. 5. Example of data fusion for enhanced SI (red contoured area): the coordinates on the axes are in meters.

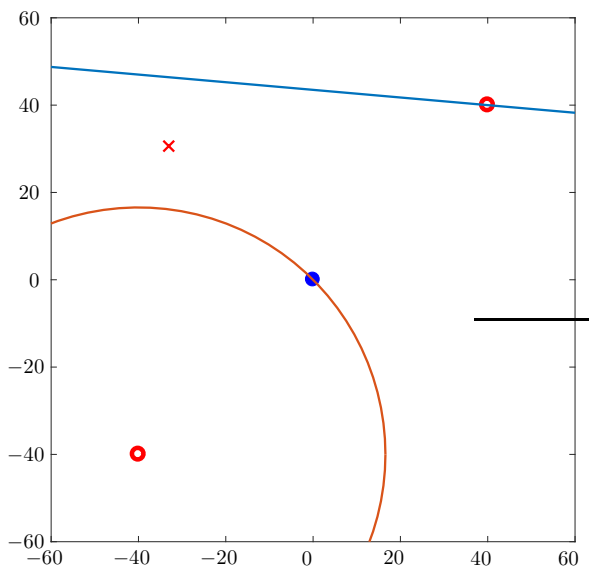

(a) Localization based on SVEs.

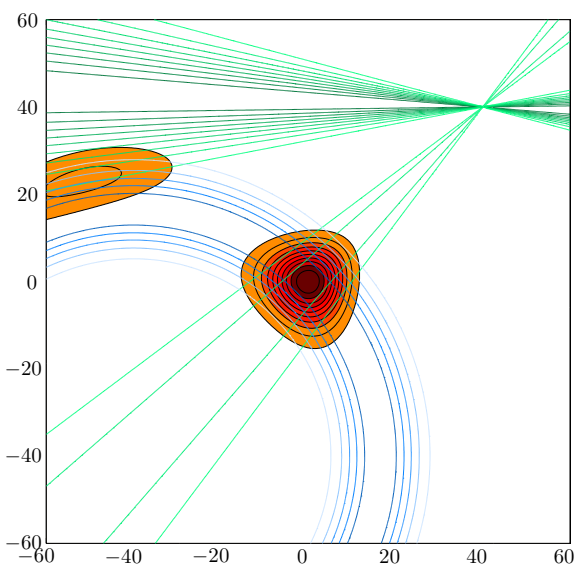

(b) Localization based on SI.

Fig. 6. Example of SVEs vs. SI-based localization: the coordinates on the axes are in meters.

of the SRI in Fig. 4(b), the SAI in Fig. 4(c), and the SCI in Fig. 4(d).

Fig. 6 shows an example of comparison between the classical and the new approach. In particular, refer to the scenario depicted in Fig. 4(a) where the bottom-left anchor provides range measurements to the target, whereas the top-right anchor provides angle measurements. Due to the harsh propagation environment, the angle measurements are affected by a bias, which results in an erroneous AE for SVE-based localization while it results in bimodal SAI for SI-based localization. These two situations are respectively depicted in Fig. 6(a) and Fig. 6(b). In particular, the cross in Fig. 6(a) represents the wrongly estimated position using the LS algorithm with DE and $\mathrm{AE}$ as inputs, whereas the dark red area in Fig. 6(b) shows that the maximum of the positional feature likelihood is near the true position. This simple example illustrates how SI on $\boldsymbol{\theta}$ provides richer information than that offered by its SVE $\hat{\boldsymbol{\theta}}$, thus improving the localization accuracy.

\section{F. Distributed Implementation}

Distributed implementation is particularly important in scenarios with networks of nodes having limited capabilities such as those in LoT. Cooperation in space and time can improve the localization accuracy. However, the use of measurements related to several agents causes information coupling [219], [220], resulting in highly interrelated inference for different agents. This fact is reflected in the concatenated arguments in the posterior distribution in (21) and (25), compared to that in (19) and (23). The optimal implementation of noncooperative approaches described in (19) and (23) can be performed in a distributed fashion since each agent can determine its own posterior distribution. On the other hand, the optimal implementation of cooperative approaches described in (21) and (25) requires a centralized implementation to determine the joint posterior distribution of all the agents.

Techniques have been developed for distributed implementation by approximating the joint posterior distribution via 


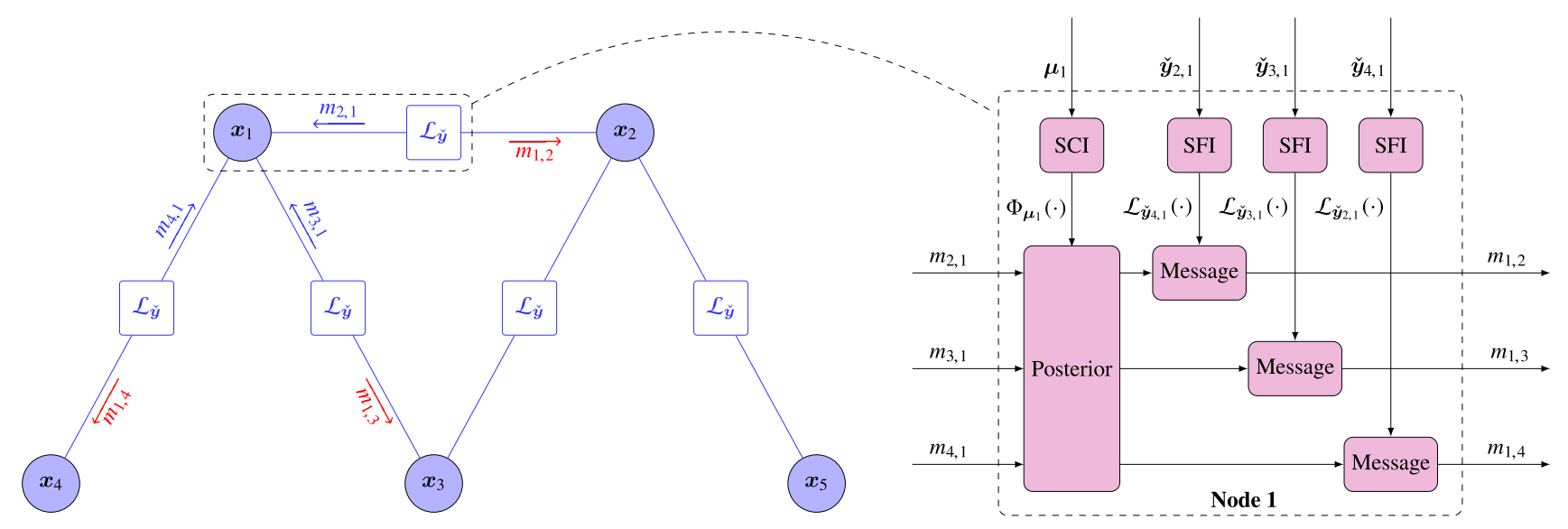

Fig. 7. Example of distributed implementation: (left) network factor graph with message passing involving node 1; and (right) computation based on different SFI and SCI inside node 1.

marginalization. For example, the loopy belief propagation technique approximates the marginal posterior distribution of each agent by disregarding the cycles in the graph describing the network connectivity [49]. Specifically, an approximate marginal posterior $\tilde{f}\left(\boldsymbol{x}_{i} \mid \check{\mathcal{Y}}\right)$ for the positional state of agent $i$, based on measurement set $\check{\mathcal{Y}}=\left\{\mathcal{Y}, \check{\boldsymbol{y}}_{i, j}\right\}$, can be obtained sequentially from $\tilde{f}\left(\boldsymbol{x}_{i} \mid \mathcal{Y}\right)$ when a new measurement $\check{\boldsymbol{y}}_{i, j}$ is available, as

$$
\tilde{f}\left(\boldsymbol{x}_{i} \mid \check{\mathcal{Y}}\right) \propto \tilde{f}\left(\boldsymbol{x}_{i} \mid \mathcal{Y}\right) m_{j, i}
$$

where

$$
m_{j, i} \propto \int \tilde{f}\left(\boldsymbol{x}_{j} \mid \mathcal{Y}\right) \mathcal{L}_{\check{\boldsymbol{y}}_{i, j}}\left(\boldsymbol{\theta}_{i, j}\right) d \boldsymbol{x}_{j}
$$

is usually referred to as message from node $j$ to node $i$. Equation (27) forms the basis for developing network messaging algorithms.

Fig. 7 shows an example of a network factor graph with messaging for distributed implementation of network localization and navigation (NLN). In particular, messages entering to and exiting from node 1 are highlighted, and the computation blocks inside node 1 are depicted.

\section{Soft Information And Performance Limits}

Fundamental limits provide performance benchmarks, which are essential for network design. In [37]-[41], a performance measure called squared position error bound has been derived as a function of the Fisher information matrix (FIM). In the following, we will derive the FIM as a function of SFI and SCI.

Let $\mathbf{x}_{\mathcal{N}_{\mathrm{a}}}^{\mathcal{N}_{\mathrm{a}}}$ be a random vector composed of positional states, for $N_{\mathrm{a}}$ agents at $N_{\mathrm{t}}$ time instants, in which the $\left(i+(n-1) N_{\mathrm{a}}\right)$ th element is $\mathbf{x}_{i}^{(n)}$. The positional state is inferred from internode measurements $\boldsymbol{y}_{i, j}^{(n)}$ related to $\boldsymbol{x}_{i}^{(n)}-\boldsymbol{x}_{j}^{(n)}$, intra-node measurements $\boldsymbol{y}_{i, i}^{(n)}$ related to $\boldsymbol{x}_{i}^{(n)}$, and a dynamic model $\boldsymbol{\mu}$ related to $\boldsymbol{x}_{i}^{(n)}-\boldsymbol{x}_{i}^{(m)}$, where $i \in \mathcal{N}_{\mathrm{a}}, j \in \mathcal{N}_{\mathrm{a}} \cup \mathcal{N}_{\mathrm{b}}$ with $j \neq i$, and $n, m \in \mathcal{N}_{\mathrm{t}}$.

According to the Fisher information inequality, an estimator $\hat{\mathbf{x}}_{i}^{(n)}$ of positional state $\mathbf{x}_{i}^{(n)}$ satisfies

$$
\mathbb{E}\left\{\left\|\hat{\mathbf{x}}_{i}^{(n)}-\mathbf{x}_{i}^{(n)}\right\|^{2}\right\} \geq \operatorname{tr}\left\{\left[\boldsymbol{J}^{-1}\right]_{i}^{(n)}\right\}
$$

where $\left[\boldsymbol{J}^{-1}\right]_{i}^{(n)}$ denotes the $\left(i+(n-1) N_{\mathrm{a}}\right)$-th $D \times D$ diagonal block in the inverse of the Bayesian FIM [221] for positional states $\mathbf{x}_{\mathcal{N}_{\mathrm{a}}}^{\mathcal{N}_{t}}$. The FIM for $\mathbf{x}_{\mathcal{N}_{\mathrm{a}}}^{\mathcal{N}_{t}}$ is given by [40]

$$
\boldsymbol{J}=\boldsymbol{J}_{\mathrm{p}}+\boldsymbol{J}_{\mathrm{s}}+\boldsymbol{J}_{\mathrm{t}}
$$

where $\boldsymbol{J}_{\mathrm{p}}$ is the FIM corresponding to prior knowledge of $\mathbf{x}_{\mathcal{N}_{\mathrm{a}}}^{\mathcal{N}_{\mathrm{a}}} ; \boldsymbol{J}_{\mathrm{S}}$ is the FIM consisting of two terms: the first term corresponds to the inter-node measurements (with anchors) and the second corresponds to the spatial cooperation (with other agents); and $\boldsymbol{J}_{\mathrm{t}}$ is the FIM consisting of two terms: the first term corresponds to the intra-node measurements (at a particular time step) and the second corresponds to temporal cooperation (between different time steps). In particular,

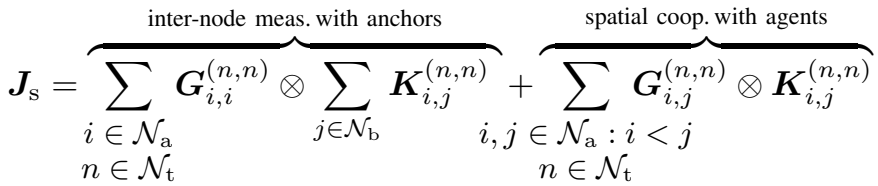

$$
\boldsymbol{J}_{\mathrm{t}}=\overbrace{\sum_{\substack{i \in \mathcal{N}_{\mathrm{a}} \\ n \in \mathcal{N}_{\mathrm{t}}}} \boldsymbol{G}_{i, i}^{(n, n)} \otimes \boldsymbol{K}_{i, i}^{(n, n)}}^{\text {intra-node meas. at one time step }}+\overbrace{\substack{i \in \mathcal{N}_{\mathrm{a}} \\ n, m \in \mathcal{N}_{\mathrm{t}}: m<n}}^{\text {temporal coop. at different time steps }}
$$

where

$$
\boldsymbol{G}_{i, j}^{(n, m)}=\left\{\begin{array}{cc}
\left(\boldsymbol{e}_{k}-\boldsymbol{e}_{l}\right)\left(\boldsymbol{e}_{k}-\boldsymbol{e}_{l}\right)^{\mathrm{T}} & \text { for }(i, n) \neq(j, m) \\
\boldsymbol{e}_{k}\left(\boldsymbol{e}_{k}\right)^{\mathrm{T}} & \text { for }(i, n)=(j, m)
\end{array}\right.
$$

in which $k=\left(i+(n-1) N_{\mathrm{a}}\right), l=\left(j+(m-1) N_{\mathrm{a}}\right)$, and $\boldsymbol{e}_{k}$ is an $N_{\mathrm{a}} N_{\mathrm{t}}$-dimensional vector with all zeros except a one at the $k$-th element. The matrices $\boldsymbol{K}$ 's in (31) will be described in the following.

Define

$$
\begin{aligned}
& \jmath_{\mathrm{sf}}\left(\boldsymbol{y} ; \boldsymbol{\theta}_{1}, \boldsymbol{\theta}_{2}, \boldsymbol{\theta}_{3}\right) \triangleq \frac{\partial \ln \mathcal{L}_{\boldsymbol{y}}(\boldsymbol{\theta})}{\partial \boldsymbol{\theta}_{1}} \frac{\partial \ln \mathcal{L}_{\boldsymbol{y}}(\boldsymbol{\theta})}{\partial \boldsymbol{\theta}_{2}^{\mathrm{T}}} \\
& \jmath_{\mathrm{sc}}\left(\boldsymbol{\mu} ; \boldsymbol{\theta}_{1}, \boldsymbol{\theta}_{2}, \boldsymbol{\theta}_{3}\right) \triangleq \frac{\partial \ln \Phi_{\boldsymbol{\mu}}(\boldsymbol{\theta})}{\partial \boldsymbol{\theta}_{1}} \frac{\partial \ln \Phi_{\boldsymbol{\mu}}(\boldsymbol{\theta})}{\partial \boldsymbol{\theta}_{2}^{\mathrm{T}}}
\end{aligned}
$$


for soft features and soft context, respectively, where the parameter vector $\boldsymbol{\theta}$ is a function of $\boldsymbol{\theta}_{1}, \boldsymbol{\theta}_{2}$, and the nuisance parameter vector $\boldsymbol{\theta}_{3}$. It is important to note that $\jmath_{\mathrm{sf}}\left(\boldsymbol{y} ; \boldsymbol{\theta}_{1}, \boldsymbol{\theta}_{2}, \boldsymbol{\theta}_{3}\right)$ is a function of $\mathcal{L}_{\boldsymbol{y}}(\boldsymbol{\theta})$ that depends on the type of measurement $\boldsymbol{y}$. Similarly, $\jmath_{\mathrm{sc}}\left(\boldsymbol{\mu} ; \boldsymbol{\theta}_{1}, \boldsymbol{\theta}_{2}, \boldsymbol{\theta}_{3}\right)$ is a function of $\Phi_{\boldsymbol{\mu}}(\boldsymbol{\theta})$ that depends on the type of contextual data $\boldsymbol{\mu}$. The matrix $\boldsymbol{K}_{i, j}^{(n, m)} \in \mathbb{R}^{D \times D}$ accounts for the pair-wise positional information related to agent $i \in \mathcal{N}_{\mathrm{a}}$ at time step $n \in \mathcal{N}_{\mathrm{t}}$ and node $j \in \mathcal{N}_{\mathrm{a}} \cup \mathcal{N}_{\mathrm{b}}$ at time step $m \in \mathcal{N}_{\mathrm{t}}$ as elaborated below.

- The $\boldsymbol{K}_{i, i}^{(n, n)}$ accounts for the information that agent $i$ obtains at time step $n$ from intra-node measurements $\boldsymbol{y}_{i, i}^{(n)}$. It can be written as

$$
\boldsymbol{K}_{i, i}^{(n, n)}=\mathbb{E}\left\{\jmath_{\mathrm{sf}}\left(\mathbf{y}_{i, i}^{(n)} ; \mathbf{x}_{i}^{(n)}, \mathbf{x}_{i}^{(n)}, \emptyset\right)\right\} .
$$

- The $\boldsymbol{K}_{i, j}^{(n, n)}$ for $j \in \mathcal{N}_{\mathrm{b}}$ accounts for the information that agent $i$ obtains at time step $n$ from inter-node measurements $\boldsymbol{y}_{i, j}^{(n)}$ with respect to anchor $j$. It can be written as

$$
\boldsymbol{K}_{i, j}^{(n, n)}=\mathbb{E}\left\{\jmath_{\mathrm{sf}}\left(\mathbf{y}_{i, j}^{(n)} ; \mathbf{x}_{i}^{(n)}, \mathbf{x}_{i}^{(n)}, \emptyset\right)\right\} .
$$

- The $\boldsymbol{K}_{i, j}^{(n, n)}$ for $j \in \mathcal{N}_{\mathrm{a}} \backslash\{i\}$ accounts for the information that agent $i$ obtains at time step $n$ from inter-node measurements $\boldsymbol{y}_{i, j}^{(n)}$ with respect to neighboring agent $j$ (i.e., spatial cooperation). It can be written as

$$
\boldsymbol{K}_{i, j}^{(n, n)}=\mathbb{E}\left\{-\jmath_{\mathrm{sf}}\left(\mathbf{y}_{i, j}^{(n)} ; \mathbf{x}_{i}^{(n)}, \mathbf{x}_{j}^{(n)}, \emptyset\right)\right\} .
$$

- The $\boldsymbol{K}_{i, i}^{(n, m)}$ accounts for the information that agent $i$ obtains at time step $n$ from its positional state at previous time step $m$ and the dynamic model $\boldsymbol{\mu}$ (i.e., temporal cooperation). ${ }^{9}$ It can be written as

$$
\boldsymbol{K}_{i, i}^{(n, m)}=\mathbb{E}\left\{-\jmath_{\mathrm{sc}}\left(\boldsymbol{\mu} ; \mathbf{x}_{i}^{(n)}, \mathbf{x}_{i}^{(m)}, \emptyset\right)\right\} .
$$

Consider a network with $N_{\mathrm{a}}=3$ agents and $N_{\mathrm{t}}=2$ time steps. The FIM can be written as in (30), in which $\boldsymbol{J}_{\mathrm{s}}$ (corresponding to spatial measurements) and $\boldsymbol{J}_{\mathrm{t}}$ (corresponding to temporal measurements) are given by (37) and (38), respectively, at the top of the next two pages. In (37), the first term represents the information coming from inter-node measurements with anchors, while the second term represents the information coming from spatial cooperation with other agents. In (38), the first term represents the information inherent in intra-node measurements at a particular time step, while the second term represents the information coming from temporal cooperation between different time steps.

\section{A. FIM from SI Functions}

The building blocks $\boldsymbol{K}_{i, j}^{(n, n)}$ of the FIM for some special cases of SFI are detailed here.

Proposition 1: Consider two-dimensional node velocity $\mathbf{v}_{i}^{(n)}$ and node position $\mathbf{p}_{i}^{(n)}$ and define the direction matrix (DM)

$$
\boldsymbol{J}_{\mathrm{dm}}(\phi) \triangleq\left[\begin{array}{cc}
\cos ^{2}(\phi) & \cos (\phi) \sin (\phi) \\
\cos (\phi) \sin (\phi) & \sin ^{2}(\phi)
\end{array}\right] .
$$

\footnotetext{
${ }^{9}$ Commonly, dynamic models provide information related to two consecutive time steps, and in those cases $\boldsymbol{K}_{i, i}^{(n, m)}=\mathbf{0}$ for $m<n-1$.
}

The blocks $\boldsymbol{K}_{i, j}^{(n, n)}$ related to speed $\left\|\mathbf{v}_{i}^{(n)}\right\|$, range $\mathrm{d}_{i, j}^{(n)}$, and angle $\alpha_{i, j}^{(n)}$ inherent in measurements involving nodes $i$ and $j$ at time instant $n$ are provided in the following.

- For intra-node measurements related to the speed,

$$
\boldsymbol{K}_{i, i}^{(n, n)}=\boldsymbol{e}_{i_{\mathrm{v}}} \boldsymbol{e}_{i_{\mathrm{v}}}^{\mathrm{T}} \otimes \mathbb{E}_{\mathbf{v}_{i}^{(n)}}\left\{\lambda_{\mathrm{S}} \mathbf{J}_{\mathrm{S}}\right\}
$$

where $i_{\mathrm{v}}$ is the velocity component index in the state vector $\mathbf{x}_{i}^{(n)}$. In (40), $\lambda_{\mathrm{s}}$ is the speed information intensity (SII) [220] and $\boldsymbol{J}_{\mathrm{s}}$ is the DM for speed measurements given by

$$
\begin{aligned}
& \lambda_{\mathrm{s}}=\mathbb{E}_{\mathbf{y}_{i, i}^{(n)} \mid \mathbf{v}_{i}^{(n)}}\left\{\jmath_{\mathrm{sf}}\left(\mathbf{y}_{i, i}^{(n)} ;\left\|\mathbf{v}_{i}^{(n)}\right\|,\left\|\mathbf{v}_{i}^{(n)}\right\|, \emptyset\right)\right\} \\
& \mathbf{J}_{\mathrm{s}}=\boldsymbol{J}_{\mathrm{dm}}\left(\alpha_{\mathrm{v}}\right)
\end{aligned}
$$

where $\alpha_{\mathrm{v}}$ is the angle between vector $\mathbf{v}_{i}^{(n)}$ and the horizontal axis.

- For inter-node measurements related to ranges,

$$
\boldsymbol{K}_{i, j}^{(n, n)}=\boldsymbol{e}_{i_{\mathrm{p}}} \boldsymbol{e}_{i_{\mathrm{p}}}^{\mathrm{T}} \otimes \mathbb{E}_{\mathbf{p}_{i}^{(n)}, \mathbf{p}_{j}^{(n)}}\left\{\lambda_{\mathrm{r}} \mathbf{J}_{\mathrm{r}}\right\}
$$

where $i_{\mathrm{p}}$ is the position component index in the state vector $\mathbf{x}_{i}^{(n)}$. In (42), $\lambda_{\mathrm{r}}$ is the range information intensity (RII) [38] and $\mathbf{J}_{\mathrm{r}}$ is the DM for range measurements given by

$$
\begin{aligned}
\lambda_{\mathrm{r}} & =\mathbb{E}_{\mathbf{y}_{i, j}^{(n)} \mid \mathbf{p}_{i}^{(n)}, \mathbf{p}_{j}^{(n)}}\left\{J_{\mathrm{sf}}\left(\mathbf{y}_{i, j}^{(n)} ; \mathrm{d}_{i, j}^{(n)}, \mathrm{d}_{i, j}^{(n)}, \emptyset\right)\right\} \\
\mathbf{J}_{\mathrm{r}} & =\boldsymbol{J}_{\mathrm{dm}}\left(\boldsymbol{\alpha}_{i, j}^{(n)}\right)
\end{aligned}
$$

where $\mathrm{d}_{i, j}^{(n)}$ is the Euclidean distance between $i$-th and $j$ th nodes, and $\alpha_{i, j}^{(n)}$ is the angle between vector $\mathbf{p}_{j}^{(n)}-\mathbf{p}_{i}^{(n)}$ and the horizontal axis.

- Finally, for inter-node measurements related to angles,

$$
\boldsymbol{K}_{i, j}^{(n, n)}=\boldsymbol{e}_{i_{\mathrm{p}}} \boldsymbol{e}_{i_{\mathrm{p}}}^{\mathrm{T}} \otimes \mathbb{E}_{\mathbf{p}_{i}^{(n)}, \mathbf{p}_{j}^{(n)}}\left\{\lambda_{\mathrm{a}} \mathbf{J}_{\mathrm{a}}\right\}
$$

where $i_{\mathrm{p}}$ is the position component index in the state vector $\mathbf{x}_{i}^{(n)}$. In (44), $\lambda_{\mathrm{a}}$ is the angle information intensity (AII) [41] and $\mathbf{J}_{\mathrm{a}}$ is the DM for angle measurements given by

$$
\begin{aligned}
& \lambda_{\mathrm{a}}=\frac{1}{\left(\mathrm{~d}_{i, j}^{(n)}\right)^{2}} \mathbb{E}_{\mathbf{y}_{i, j}^{(n)} \mid \mathbf{p}_{i}^{(n)}, \mathbf{p}_{j}^{(n)}}\left\{\jmath_{\mathrm{sf}}\left(\mathbf{y}_{i, j}^{(n)} ; \boldsymbol{\alpha}_{i, j}^{(n)}, \boldsymbol{\alpha}_{i, j}^{(n)}, \emptyset\right)\right\} \\
& \mathbf{J}_{\mathrm{a}}=\boldsymbol{J}_{\mathrm{dm}}\left(\alpha_{i, j}^{(n)}+\frac{\pi}{2}\right) .
\end{aligned}
$$

Proof: In what follows, we provide the proof for the case of intra-node measurements $\boldsymbol{y}_{i, i}^{(n)}$ related to speed $\left\|\boldsymbol{v}_{i}^{(n)}\right\|$; the other two cases can be obtained analogously. From (33),

$$
\boldsymbol{K}_{i, i}^{(n, n)}=\mathbb{E}_{\mathbf{y}_{i, i}^{(n)}, \mathbf{v}_{i}^{(n)}}\left\{\jmath_{\mathrm{sf}}\left(\mathbf{y}_{i, i}^{(n)} ; \mathbf{x}_{i}^{(n)}, \mathbf{x}_{i}^{(n)}, \emptyset\right)\right\}
$$

which results in

$$
\boldsymbol{K}_{i, i}^{(n, n)}=\boldsymbol{e}_{i_{\mathrm{v}}} \boldsymbol{e}_{i_{\mathrm{v}}}^{\mathrm{T}} \otimes \mathbb{E}_{\mathbf{y}_{i, i}^{(n)}, \mathbf{v}_{i}^{(n)}}\left\{\jmath_{\mathrm{sf}}\left(\mathbf{y}_{i, i}^{(n)} ; \mathbf{v}_{i}^{(n)}, \mathbf{v}_{i}^{(n)}, \emptyset\right)\right\}
$$




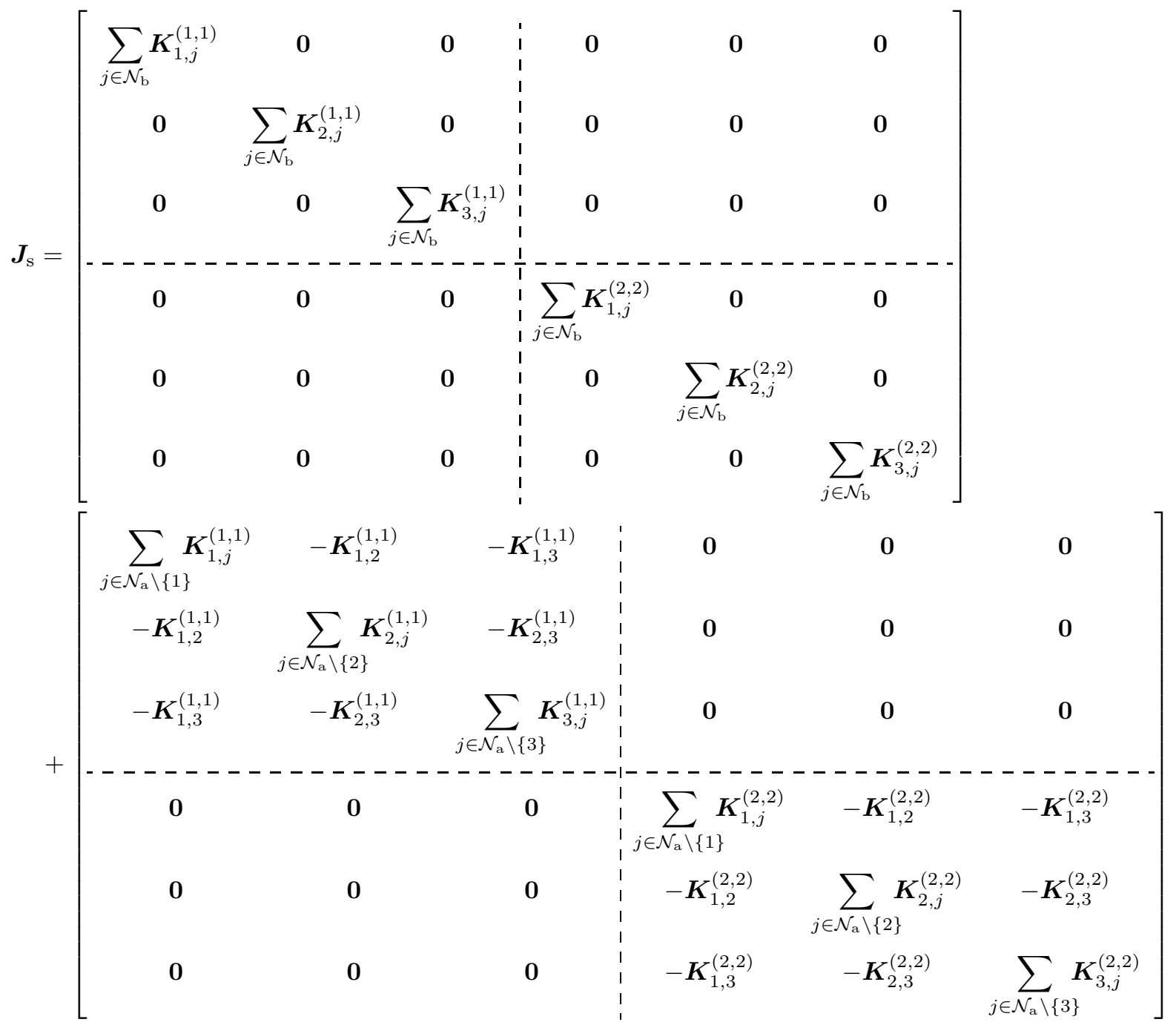

because $\boldsymbol{y}_{i, i}^{(n)}$ are measurements related to velocity. By using the chain rule for derivatives,

$$
\begin{aligned}
\jmath_{\mathrm{sf}}\left(\boldsymbol{y}_{i, i}^{(n)} ; \boldsymbol{v}_{i}^{(n)}, \boldsymbol{v}_{i}^{(n)}, \emptyset\right)= & \jmath_{\mathrm{sf}}\left(\boldsymbol{y}_{i, i}^{(n)} ;\left\|\boldsymbol{v}_{i}^{(n)}\right\|,\left\|\boldsymbol{v}_{i}^{(n)}\right\|, \emptyset\right) \\
& \times \frac{\partial\left\|\boldsymbol{v}_{i}^{(n)}\right\|}{\partial \boldsymbol{v}_{i}^{(n)}}\left(\frac{\partial\left\|\boldsymbol{v}_{i}^{(n)}\right\|}{\partial \boldsymbol{v}_{i}^{(n)}}\right)^{\mathrm{T}}
\end{aligned}
$$

since $\boldsymbol{y}_{i, i}^{(n)}$ are measurements related to speed.

Performing the expectations in (46) using the law of iterated expectations as $\mathbb{E}_{\mathbf{y}_{i, i}^{(n)}, \mathbf{v}_{i}^{(n)}}\{\cdot\}=\mathbb{E}_{\mathbf{v}_{i}^{(n)}}\left\{\mathbb{E}_{\mathbf{y}_{i, i}^{(n)} \mid \mathbf{v}_{i}^{(n)}}\{\cdot\}\right\}$, we obtain (40) in which $\lambda_{\mathrm{s}}$ in (41a) and $\boldsymbol{J}_{\mathrm{s}}$ in (41b) result respectively from the expectation of the first and second term in the right side of (47).

Remark 2: Proposition 1 indicates that measurements related to the speed provide information with intensity $\lambda_{\mathrm{s}}$ in the direction of $\mathbf{v}_{i}^{(n)}$, since $\mathbf{J}_{\mathrm{s}}$ has only one eigenvector associated with non-zero eigenvalue in such direction. Similarly, measurements related to the range provide information with intensity $\lambda_{\mathrm{r}}$ in the direction of $\mathbf{p}_{j}^{(n)}-\mathbf{p}_{i}^{(n)}$, since $\mathbf{J}_{\mathrm{r}}$ has only one eigenvector associated with non-zero eigenvalue in such direction. Finally, measurements related to the angle provide information with intensity $\lambda_{\mathrm{a}}$ in the direction orthogonal to $\mathbf{p}_{j}^{(n)}-\mathbf{p}_{i}^{(n)}$, since $\mathbf{J}_{\mathrm{a}}$ has only one eigenvector associated with non-zero eigenvalue in such direction.

\section{B. FIM in Harsh Propagation Environments}

This section provides the SFI from inter-node measurements when a detector for NLOS propagation conditions is employed, as described in Sections II-A and III-D.

Proposition 2: Consider the inter-node measurement $\boldsymbol{y}_{i, j}^{(n, n)}=\left[z^{\mathrm{T}}, \delta\right]^{\mathrm{T}}$ where $z$ is a measurement related to a feature $\theta$ and $\delta$ is the NLOS detector outcome as described in Section II-A. When $\mathcal{L}_{\boldsymbol{y}}(\boldsymbol{\theta})$ follows (13), and $z$ follows the measurement model in (14) and (15) with $\sigma_{\mathrm{LOS}}=\sigma_{\mathrm{NLOS}}=$ $\sigma$, and $\epsilon_{\mathrm{LOS}}=\epsilon_{\mathrm{NLOS}}=\epsilon$, then the FIM block corresponding to $\mathbf{y}_{i, j}^{(n, n)}$ is given by

$$
\boldsymbol{K}_{i, j}^{(n, n)}=\boldsymbol{e}_{i_{\mathrm{p}}} \boldsymbol{e}_{i_{\mathrm{p}}}^{\mathrm{T}} \otimes \mathbb{E}_{\theta}\left\{\lambda_{\theta} \boldsymbol{J}_{\theta}\right\}
$$




$\begin{aligned} & \boldsymbol{J}_{\mathrm{t}}= {\left[\begin{array}{ccc:ccc}\boldsymbol{K}_{1,1}^{(1,1)} & \mathbf{0} & \mathbf{0} & \mathbf{0} & \mathbf{0} & \mathbf{0} \\ \mathbf{0} & \boldsymbol{K}_{2,2}^{(1,1)} & \mathbf{0} & \mathbf{0} & \mathbf{0} & \mathbf{0} \\ \mathbf{0} & \mathbf{0} & \boldsymbol{K}_{3,3}^{(1,1)} & \mathbf{0} & \mathbf{0} & \mathbf{0} \\ \hdashline \mathbf{0} & \mathbf{0} & \mathbf{0} & \boldsymbol{K}_{1,1}^{(2,2)} & \mathbf{0} & \mathbf{0} \\ \mathbf{0} & \mathbf{0} & \mathbf{0} & \mathbf{0} & \boldsymbol{K}_{2,2}^{(2,2)} & \mathbf{0} \\ \mathbf{0} & \mathbf{0} & \mathbf{0} & \mathbf{0} & \mathbf{0} & \boldsymbol{K}_{3,3}^{(2,2)}\end{array}\right] } \\ &+\left[\begin{array}{cccc:ccc}\boldsymbol{K}_{1,1}^{(2,1)} & \mathbf{0} & & \mathbf{0} & -\boldsymbol{K}_{1,1}^{(2,1)} & \mathbf{0} & \mathbf{0} \\ \mathbf{0} & \boldsymbol{K}_{2,2}^{(2,1)} & \mathbf{0} & \mathbf{0} & -\boldsymbol{K}_{2,2}^{(2,1)} & \mathbf{0} \\ \mathbf{0} & \mathbf{0} & \boldsymbol{K}_{3,3}^{(2,1)} & \mathbf{0} & \mathbf{0} & -\boldsymbol{K}_{3,3}^{(2,1)} \\ \hdashline \boldsymbol{K}_{1,1}^{(2,1)} & \mathbf{0} & \mathbf{0} & \boldsymbol{K}_{1,1}^{(2,1)} & \mathbf{0} & \mathbf{0} \\ \mathbf{0} & -\boldsymbol{K}_{2,2}^{(2,1)} & \mathbf{0} & \mathbf{0} & \boldsymbol{K}_{2,2}^{(2,1)} & \mathbf{0} \\ \mathbf{0} & \mathbf{0} & -\boldsymbol{K}_{3,3}^{(2,1)} & \mathbf{0} & \mathbf{0} & \boldsymbol{K}_{3,3}^{(2,1)}\end{array}\right]\end{aligned}$

where $\lambda_{\theta}$ and $\boldsymbol{J}_{\theta}$ have instantiations

$$
\begin{aligned}
\lambda_{\theta}= & \frac{1}{\sigma^{4}}\left[\mathbb{E}_{\mathbf{z} \mid \theta}\left\{\left(\mathbf{z}-\theta-\mathbf{\chi}_{0}\right)^{2} \mid \delta=0\right\} \mathbb{P}\{\delta=0\}\right. \\
& \left.+\mathbb{E}_{\mathbf{z} \mid \theta}\left\{\left(\mathbf{z}-\theta-\boldsymbol{\chi}_{1}\right)^{2} \mid \delta=1\right\} \mathbb{P}\{\delta=1\}\right] \\
\boldsymbol{J}_{\theta}= & \left(\frac{\partial \theta}{\partial \boldsymbol{p}_{i}^{(n)}}\right)\left(\frac{\partial \theta}{\partial \boldsymbol{p}_{i}^{(n)}}\right)^{\mathrm{T}}
\end{aligned}
$$

as well as $\chi_{0}$ and $\chi_{1}$ have instantiations

$$
\begin{aligned}
& \chi_{0}=\frac{\beta \epsilon \varphi\left(\theta ; z-\beta, \sigma^{2}\right)}{(1-\epsilon) \varphi\left(\theta ; z, \sigma^{2}\right)+\epsilon \varphi\left(\theta ; z-\beta, \sigma^{2}\right)} \\
& \chi_{1}=\frac{\beta(1-\epsilon) \varphi\left(\theta ; z-\beta, \sigma^{2}\right)}{\epsilon \varphi\left(\theta ; z, \sigma^{2}\right)+(1-\epsilon) \varphi\left(\theta ; z-\beta, \sigma^{2}\right)} .
\end{aligned}
$$

Proof: Equation (48) is obtained from Proposition 1 and the fact that

$$
\frac{\partial \ln \mathcal{L}_{\boldsymbol{y}_{i, j}^{(n)}}(\theta)}{\partial \theta}= \begin{cases}\frac{1}{\sigma^{2}}\left(z-\theta-\chi_{0}\right) & \text { for } \delta=0 \\ \frac{1}{\sigma^{2}}\left(z-\theta-\chi_{1}\right) & \text { for } \delta=1 .\end{cases}
$$

Remark 3: For $\epsilon=0$ and $\mathbb{P}\{\delta=0\}=1$ (LOS scenarios with totally reliable NLOS detector), the term $\chi_{0}=0$ and (48) results in the known expression for LOS scenarios (i.e., $\lambda=1 / \sigma^{2}$ ) [38]. Moreover, the two Gaussian PDFs $\varphi(\cdot)$ in (50a) and (50b) have negligible overlap for $\beta \gg \sigma$, as well as $\chi_{0} \approx \chi_{1} \approx 0$ (resp. $\chi_{0} \approx \chi_{1} \approx \beta$ ) when $\mathrm{z}$ has mean $\theta$ (resp. $\theta+\beta$ ) and standard deviation $\sigma$. Therefore, for $\beta \gg \sigma$ (48) approximates the $\boldsymbol{K}_{i, j}^{(n, n)}$ for LOS scenarios with totally reliable NLOS detector (i.e., $\lambda \approx 1 / \sigma^{2}$ ), independently of the detector reliability $\epsilon$.

\section{LEARNING SOFT INFORMATION}

Using a Bayesian formulation, the SFI can be determined based on a joint distribution function, referred to as generative model, of the positional feature together with measurements and contextual data. For instance, the SFI inherent in a measurement vector $\boldsymbol{y}$ related to feature $\boldsymbol{\theta}$ can be determined as $\mathcal{L}_{\boldsymbol{y}}(\boldsymbol{\theta}) \propto f_{\mathbf{y}, \theta}(\boldsymbol{y}, \boldsymbol{\theta})$, in the absence of prior information on $\boldsymbol{\theta}$, or as $\mathcal{L}_{\boldsymbol{y}}(\boldsymbol{\theta})=f_{\mathbf{y}, \theta}(\boldsymbol{y}, \boldsymbol{\theta}) / f_{\theta}(\boldsymbol{\theta})$, in the presence of prior information on $\boldsymbol{\theta}$ [55]. Analogously, SCI inherent in contextual data $\boldsymbol{\mu}$ related to acceleration $\boldsymbol{a}$, angular velocity $\boldsymbol{\omega}$, and velocity $\boldsymbol{v}$ can be obtained as $\Phi_{\boldsymbol{\mu}}(\boldsymbol{a}, \boldsymbol{\omega}, \boldsymbol{v}) \propto f(\boldsymbol{a}, \boldsymbol{\omega}, \boldsymbol{v} ; \boldsymbol{\mu})$ [153].

In simple scenarios, the generative model can be accurately determined based on the relation between measurements, positional features, and contextual data. In more complex scenarios, finding an accurate generative model is challenging and it is preferable to learn it using measurements, positional features, and contextual data by a process commonly known as density estimation [222]-[224]. In particular, the SI can be determined by a two-phase algorithm:

(i) off-line phase where the approximate of the generative model is determined from measurements, positional features, and context data; and

(ii) on-line phase where the SFI and SCI for each new measurement are determined based on the generative model learned in the previous phase. 


\section{Algorithm 1 - SFI estimation with dimensionality reduction Off-line Phase}

1: Acquire training data $\left\{\boldsymbol{y}^{(k)}, \boldsymbol{\theta}^{(k)}\right\}_{k \in \mathcal{N}_{\text {train }}}$ through a measurement campaign realized in time steps indexed by $\mathcal{N}_{\text {train }}$.

2: Perform dimensionality reduction of training data:

$$
\left\{\boldsymbol{y}^{(k)}, \boldsymbol{\theta}^{(k)}\right\}_{k \in \mathcal{N}_{\text {train }}} \rightarrow\left\{\psi\left(\boldsymbol{y}^{(k)}\right), \boldsymbol{\theta}^{(k)}\right\}_{k \in \mathcal{N}_{\text {train }}} .
$$

3: Determine an approximate generative model $\tilde{f}(\psi(\boldsymbol{y}), \boldsymbol{\theta})$.

4: Store the approximate generative model.

\section{On-line Phase}

1: for $k \geq 0$ do

2: Acquire a new measurement vector $\boldsymbol{y}^{(k)}$ at time $t_{k}$.

3: Perform dimensionality reduction of the new measurement vector:

$$
\boldsymbol{y}^{(k)} \rightarrow \psi\left(\boldsymbol{y}^{(k)}\right) .
$$

4: Determine the SFI of the reduced measurement vector $\psi\left(\boldsymbol{y}^{(k)}\right)$ using the stored generative model as

$$
\mathcal{L}_{\psi\left(\boldsymbol{y}^{(k)}\right)}(\boldsymbol{\theta})=\tilde{f}\left(\psi\left(\boldsymbol{y}^{(k)}\right), \boldsymbol{\theta}\right) .
$$

\section{5: end for}

The off-line phase determines generative models for environments similar to (but not necessarily the same as) those where the localization network will operate (i.e., where the on-line phase is performed). The exploitation of SFI and SCI has a complexity that depends on the generative model learned during the off-line phase; therefore, constraints on the computation and communication capabilities of nodes call for tractable and parsimonious generative models. Techniques such as belief condensation [53], which approximate complicated distributions by combination of simple ones, can enable the use of tractable generative models for efficient implementation of SI-based localization.

\section{A. SI from Reduced Data Set}

Determining the generative model from training data can be difficult, especially for measurement vectors with high dimensionality (e.g., waveform samples with fine time-delay resolution) [55]. Therefore, dimensionality reduction is crucial for efficient learning of SFI. Such a dimensionality reduction step can be described as a function $\psi(\cdot)$ that transforms a measurement vector $\boldsymbol{y} \in \mathbb{R}^{M}$ into $\psi(\boldsymbol{y}) \in \mathbb{R}^{M^{\prime}}$ with $M^{\prime}$ significantly smaller than $M$. The dimensionality reduction may not necessarily involve SVEs, while SVEs can be thought of as a specific type of dimensionality reduction. While the proposed SI-based approach can be used for any type of measurement, the dimensionality reduction and generative model learning techniques are technology-dependent.

An algorithm for estimating the SFI with dimensionality reduction is composed of two phases (see Algorithm 1): an off-line phase in which dimensionality reduction $\psi(\cdot)$ is performed ${ }^{10}$ and generative models are determined based on training measurements; and an on-line phase in which the SFI

\footnotetext{
${ }^{10}$ Clearly, $\psi\left(\boldsymbol{y}^{(k)}\right)=\boldsymbol{y}^{(k)}$ in the absence of dimensionality reduction.
}

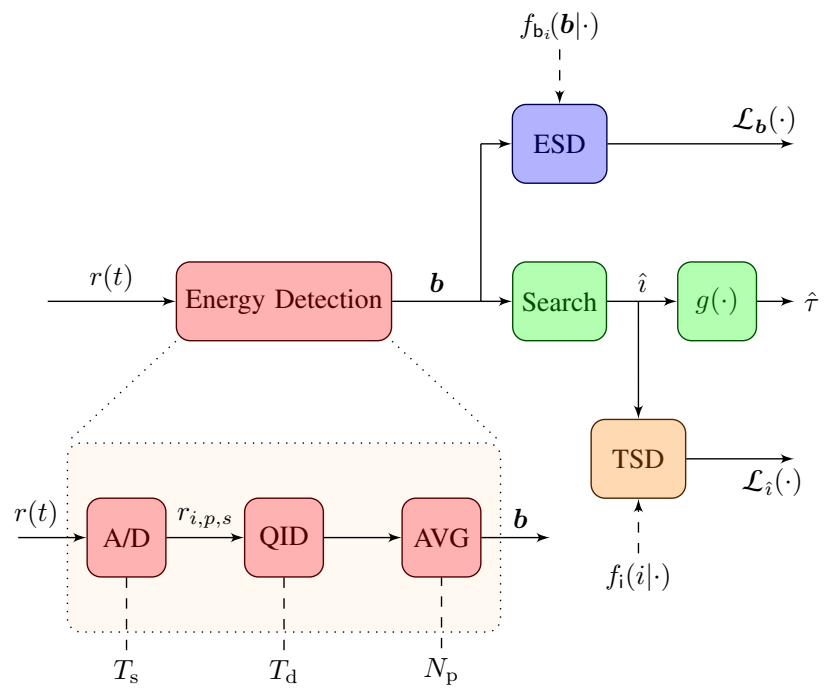

(a) Energy detection-based range information: use of $\boldsymbol{b}$ for obtaining the DE and the SRI based on energy-based soft-decision (ESD) and threshold-based soft-decision (TSD).

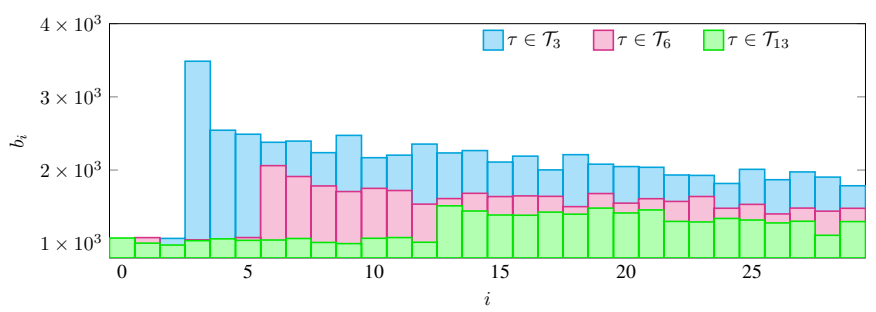

(b) Examples of bin outcomes for different wireless conditions: $\tau \in \mathcal{T}_{n}$ indicates TOA $\tau$ within the $n$-th dwell time.

Fig. 8. Range information from energy detection.

is learned from the generative model and each measurement collected during operation.

Various techniques can be used for performing dimensionality reduction and determining the generative model. Unsupervised machine learning techniques provide ways to learn SFI. In particular, SRI learning is addressed in [55], where techniques for dimensionality reduction based on physical features, principal component analysis (PCA), and Laplacian eigenmap are introduced, as well as techniques for determining generative models based on Fisher-Wald setting and kernel density estimation are presented.

Energy detection-based techniques are often used to determine information on the TOA $\tau$ of the received signal, which is related to the distance between transmitter and receiver [115]. In such a case, the SRI can be obtained based on the distribution function of the $N_{\text {bin }}$ energy samples (bins) at the energy detector output (see Fig. 8). In [51], a model for wideband ranging was proposed together with the PDF of each energy bin $\mathrm{b}_{i}, f_{\mathrm{b}_{i}}\left(b_{i} \mid \tau, \boldsymbol{\eta}_{\mathrm{h}}, \boldsymbol{\eta}_{\mathrm{d}}\right)$, and the probability mass function (PMF) of the selected bin i, $f_{\mathrm{i}}\left(\hat{\imath} \mid \tau, \boldsymbol{\eta}_{\mathrm{h}}, \boldsymbol{\eta}_{\mathrm{d}}\right)$ for a variety of ranging algorithms, where $\boldsymbol{\eta}_{\mathrm{h}}$ and $\boldsymbol{\eta}_{\mathrm{d}}$ are parameter vectors representing the wireless channel and the energy detector, respectively. Such a model is essential for 
TABLE I

TIME- AND AMPLITUDE-BASED MEASUREMENT SELECTION FEATURES

\begin{tabular}{lll}
\hline Sample distributions/statistics & Time-based selection features & Amplitude-based selection features \\
\hline$\tilde{f}_{i j}(q)=\nu_{i j}^{(q)}\left(\sum_{q=0}^{N_{\mathrm{d}}-1} \nu_{i j}^{(q)}\right)^{-1}$ & $I Q R_{i j}=\tilde{F}_{i j}^{-1}(0.75)-\tilde{F}_{i j}^{-1}(0.25)$ & $M_{i j}=\max _{q} \nu_{i j}^{(q)}$ \\
$\tilde{F}_{i j}(x)=\sum_{q \leq x} \tilde{f}_{i j}(q)$ & $\tilde{\sigma}_{i j}^{2}=\tilde{\mu}_{i j}^{(2)}$ & $s_{i j}^{2}=\frac{1}{N_{\mathrm{d}}-1} \sum_{q=0}^{N_{\mathrm{d}}-1}\left[\nu_{i j}^{(q)}-\left(\frac{1}{N_{\mathrm{d}}} \sum_{q=0}^{N_{\mathrm{d}}-1} \nu_{i j}^{(q)}\right)\right]^{2}$ \\
$m_{i j}=\sum_{q=0}^{N_{\mathrm{d}}-1} q \tilde{f}_{i j}(q)$ & $\tilde{\kappa}_{i j}=\frac{\tilde{\mu}_{i j}^{(4)}}{\left(\tilde{\mu}_{i j}^{(2)}\right)^{2}}$ & $r_{i j}=\left|\max _{q} \nu_{i j}^{(q)}-\min _{q} \nu_{i j}^{(q)}\right|$ \\
$\tilde{\mu}_{i j}^{(n)}=\sum_{q=0}^{N_{\mathrm{d}}-1}\left(q-m_{i j}\right)^{n} \tilde{f}_{i j}(q)$ & $\tilde{\chi}_{i j}=\frac{\tilde{\mu}_{i j}^{(3)}}{\left(\tilde{\mu}_{i j}^{(2)}\right)^{3 / 2}}$ & $c_{i j}=\frac{1}{N_{\mathrm{d}}\left(s_{i j}^{2}\right)^{3 / 2}} \sum_{q=0}^{N_{\mathrm{d}}-1}\left[\nu_{i j}^{(q)}-\frac{1}{N_{\mathrm{d}}}\left(\sum_{q=0}^{N_{\mathrm{d}}-1} \nu_{i j}^{(q)}\right)\right]^{3}$
\end{tabular}

obtaining the SRI from the energy detector output samples. The size of the observation set is important for computation and communication of the SRI; therefore, alternative methods based on reduced data sets of the observations were proposed in [225]. In particular, the SRI for a given observation of the energy bins $\boldsymbol{b}$ can be written as ${ }^{11}$

$$
\mathcal{L}_{\boldsymbol{b}}(\tau)=\prod_{i=0}^{N_{\mathrm{bin}}-1} f_{\mathrm{b}_{i}}\left(b_{i} \mid \tau, \boldsymbol{\eta}_{\mathrm{h}}, \boldsymbol{\eta}_{\mathrm{d}}\right) .
$$

This is referred to as energy-based soft-decision (ESD) (Fig. 8(a)) and is obtained from a data set of size $N_{\text {bin }}$ (reduced by a factor $N_{\mathrm{sb}}$, the number of samples per bin, compared to SRI obtained from the complete set of received waveform samples). SRI can also be obtained from the PMF of the selected bin index as

$$
\mathcal{L}_{\hat{\imath}}(\tau)=f_{\mathrm{i}}\left(\hat{\imath} \mid \tau, \boldsymbol{\eta}_{\mathrm{h}}, \boldsymbol{\eta}_{\mathrm{d}}\right) .
$$

This is referred to as threshold-based soft-decision (TSD) (Fig. 8(a)) and is obtained from a data set of size one (reduced by a factor $N_{\text {sb }} N_{\text {bin }}$ compared to SRI obtained from the complete set of received waveform samples). The SRI provided by the likelihood functions (51) or (52) can be used for SI-based localization. ${ }^{12}$

\section{B. Selection of Representative Measurements}

Accurate LoT is challenging in harsh propagation conditions, where multipath, clutter, and signal obstructions can give erroneous measurements that are not representative of the positional states. These measurements, also called nonrepresentative outliers [226], can adversely impact the localization performance [131]-[133]. In the context of LoT, it is particularly important to develop low-complexity techniques that select a measurement subset $\mathcal{Y}_{\text {sel }} \subseteq \mathcal{Y}$ containing the measurements that are more representative of positional states.

We now describe measurement selection techniques that do not require the knowledge of the wireless environment and rely

\footnotetext{
${ }^{11}$ The likelihood of the TOA is strictly related to that of the distance.

${ }^{12}$ The SRI can also be used for SVE-based localization, in fact the maximum of the SRI enables the determination of DEs for position inference in classical two-stage localization.
}

only on features extracted from received waveform samples [130]. Consider a vector

$$
\boldsymbol{\nu}_{i j}=\left[\nu_{i j}^{(0)}, \nu_{i j}^{(1)}, \ldots, \nu_{i j}^{\left(N_{\mathrm{d}}-1\right)}\right]^{\mathrm{T}}
$$

of $N_{\mathrm{d}}$ indicator samples for the pair $(i, j)$. In the case of energy detection, the $\nu_{i j}^{(q)}$ is related to the energy of the samples within the $q$-th time interval (dwell time). Table I presents temporal and amplitude features based on the vector $\nu_{i j}$ for selecting the observations that are representative of the nodes positions (i.e., less affected by multipath, noise, and obstructionloss). In particular, time-based selection features are: interquartile range $I Q R_{i j}$, variance $\tilde{\sigma}_{i j}^{2}$, kurtosis $\tilde{\kappa}_{i j}$, and skewness $\tilde{\chi}_{i j}$. Amplitude-based selection features are: maximum value $M_{i j}$, sample variance $s_{i j}^{2}$, sample range $r_{i j}$, and sample skewness $c_{i j}$. For each scenario, it is essential to choose the selection feature $h\left(\boldsymbol{\nu}_{i j}\right) \in\left\{I Q R_{i j}, \tilde{\sigma}_{i j}^{2}, \tilde{\kappa}_{i j}, \tilde{\chi}_{i j}, M_{i j}, s_{i j}^{2}, r_{i j}, c_{i j}\right\}$ or a combination of them based on its relationship with the localization performance [130].

\section{CASe Studies}

This section compares the performance of SVE-based, DP, and SI-based techniques in two case studies corresponding to the following scenarios:

- noisy features and NLOS detection; and

- IEEE 802.15.4 a standard.

In each scenario, measurements are obtained in different wireless environments.

Before delving into the performance comparison in each case study (CS), a discussion on the complexity of the SVEbased, DP, and SI-based techniques is given. The SVE-based technique does not require an off-line phase (training) and relies only on a single value per measurement. The DP technique requires prior knowledge of the channel model. If such a model is unknown, then DP uses an off-line phase to estimate the channel response (from multiple received waveforms for each anchor-agent distance).$^{13}$ The SI-based technique requires an off-line phase to determine a generative model for the SFI (however, it does not require multiple received waveforms for each anchor-agent distance) [55]. In the on-line phase,

\footnotetext{
${ }^{13}$ In the IEEE 802.15 .4 a scenario, waveforms are processed in time domain and a covariance matrix is obtained for each anchor-agent distance [141].
} 


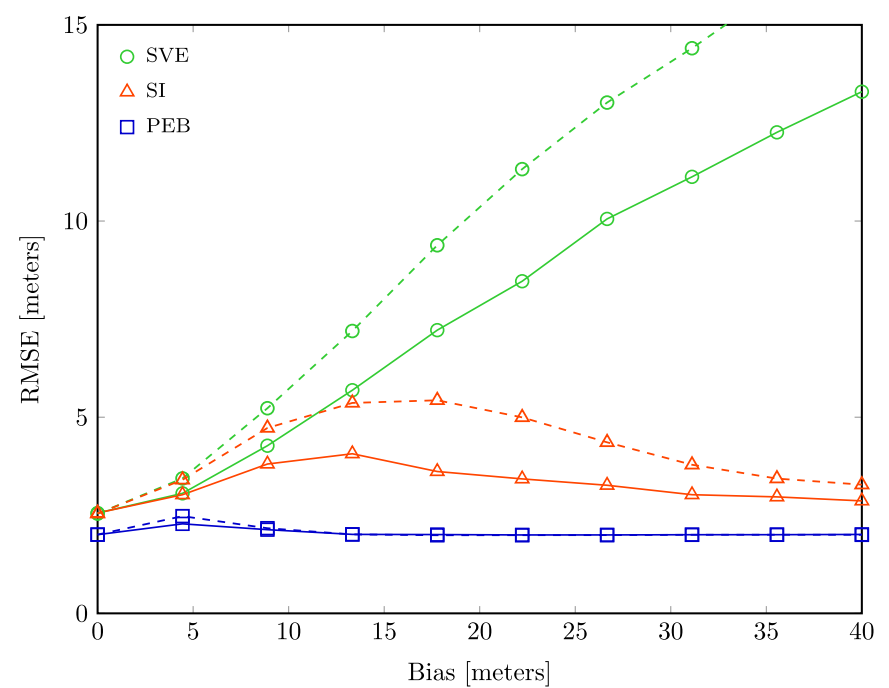

Fig. 9. Localization performance based on range measurements as a function of obstructed propagation bias for $\epsilon=0.1$ (solid) and 0.2 (dashed). The performance of SVE-based localization (green circle) and SI-based localization (red triangle) as well as the PEB (blue square) are shown.

DP technique computes a likelihood function that depends on the entire received waveform, resulting in high computational complexity, whereas the SI-based technique benefits from a dimensionality reduction step resulting in significantly lower complexity despite the moderate information loss.

\section{A. CS-I: Measurements Based on Noisy Features and NLOS Detection}

Consider a network in a 100 meters by 100 meters area with four anchors and a varying number of agents all randomly deployed therein. This case study compares the performance of SI-based and SVE-based localization, in terms of rootmean-square error (RMSE), together with the position error bound (PEB) as a benchmark. ${ }^{14}$ The measurement set is composed of noisy features and NLOS detector output. The noisy features are related to ranges and/or angles according to (14) and (15), and the NLOS detector error follows (1). Specifically, we consider $\epsilon_{\mathrm{NLOS}}=\epsilon_{\mathrm{LOS}}=\epsilon, p_{\mathrm{NLOS}}=0.4$, and $\sigma_{\mathrm{NLOS}}=\sigma_{\mathrm{LOS}}=\sigma$ with $\sigma=2$ meters for range measurements and $\sigma=2$ degrees for angle measurements. We compare SVE-based and SI-based techniques using the same measurements for inferring agent positions. In particular, SVE-based localization employs Gaussian measurement model with mean and variance given respectively by (2) and (3). On the other hand, SI-based localization exploits SFI according to (13) and (16) for inferring agent positions based on the posterior distribution given by (19), (21), (23), or (25) for different levels of spatial and temporal cooperation.

Fig. 9 shows the localization performance based on range measurements as a function of the obstructed propagation bias $\beta$ for different values of $\epsilon$. Notice that SRI-based localization provides significant performance improvement and robustness

\footnotetext{
${ }^{14} \mathrm{PEB}$ is the square root of the right side of (29), which is independent of the specific localization technique used and serves as a benchmark for the MSE of unbiased position estimators.
}

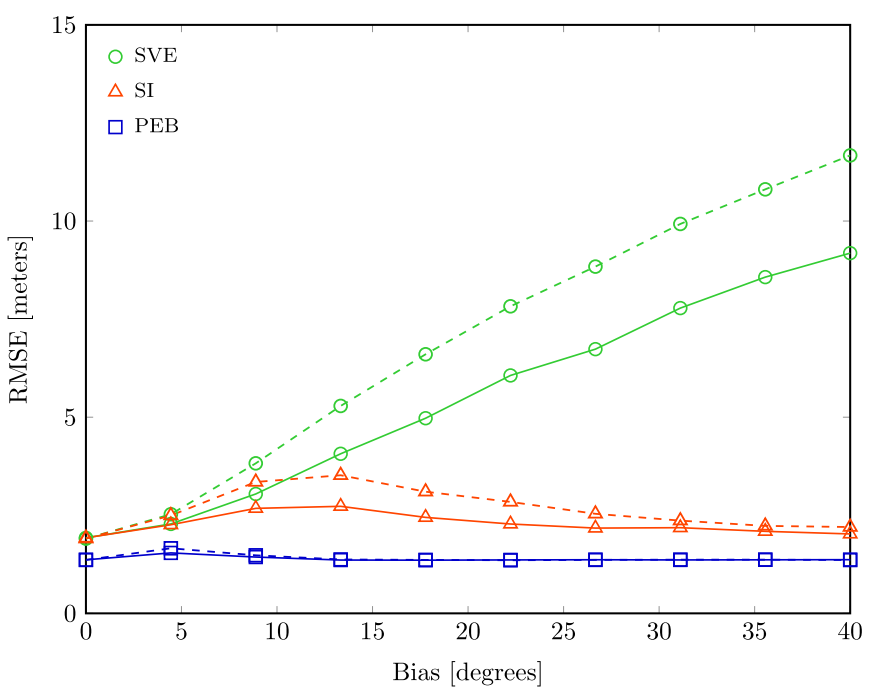

Fig. 10. Localization performance based on angle measurements as a function of obstructed propagation bias for $\epsilon=0.1$ (solid) and 0.2 (dashed). The performance of SVE-based localization (green circle) and SI-based localization (red triangle) as well as the PEB (blue square) are shown.

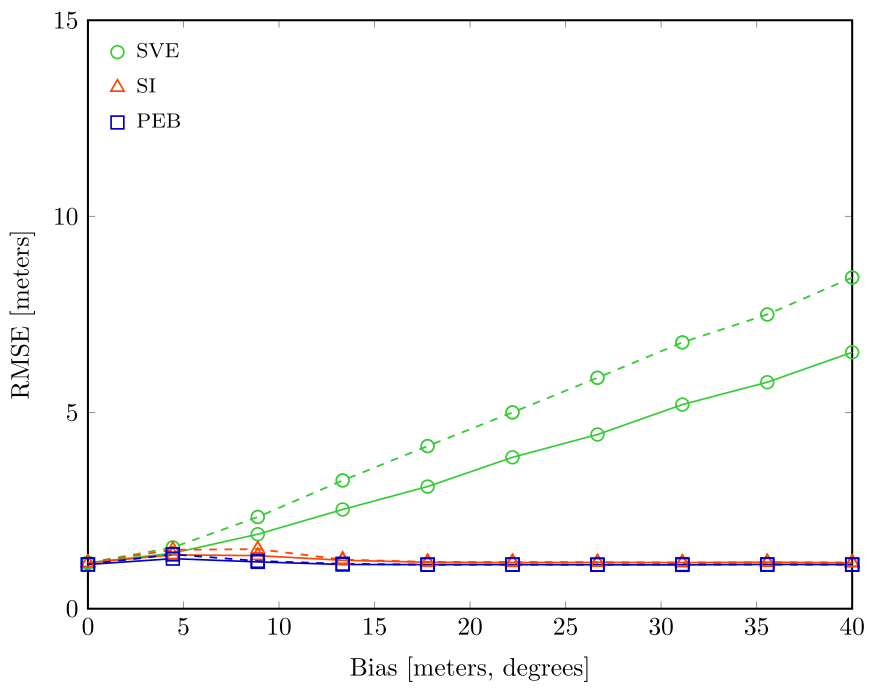

Fig. 11. Localization performance based on range and angle measurements as a function of obstructed propagation bias for $\epsilon=0.1$ (solid) and 0.2 (dashed). The performance of SVE-based localization (green circle) and SIbased localization (red triangle) as well as the PEB (blue square) are shown.

to NLOS detection errors compared to DE-based localization. Also observe that exploiting SRI enables the filling of most of the performance gap between DE-based localization and PEB. Similar observations can be made from Fig. 10, which shows the localization performance based on angle measurements for SAI-based and AE-based localization.

Now, consider the fusion of range and angle measurements. Fig. 11 shows the localization performance as a function of the obstructed propagation bias $\beta$ for different values of $\epsilon .^{15}$ Notice that SI-based localization exploiting both SRI and SAI provides significant performance improvement and robustness to NLOS detection errors compared to SVE-based localization

\footnotetext{
${ }^{15}$ For example, $\beta=20$ indicates that the bias on range measurements is of 20 meters and the bias on angle measurements is of 20 degrees.
} 


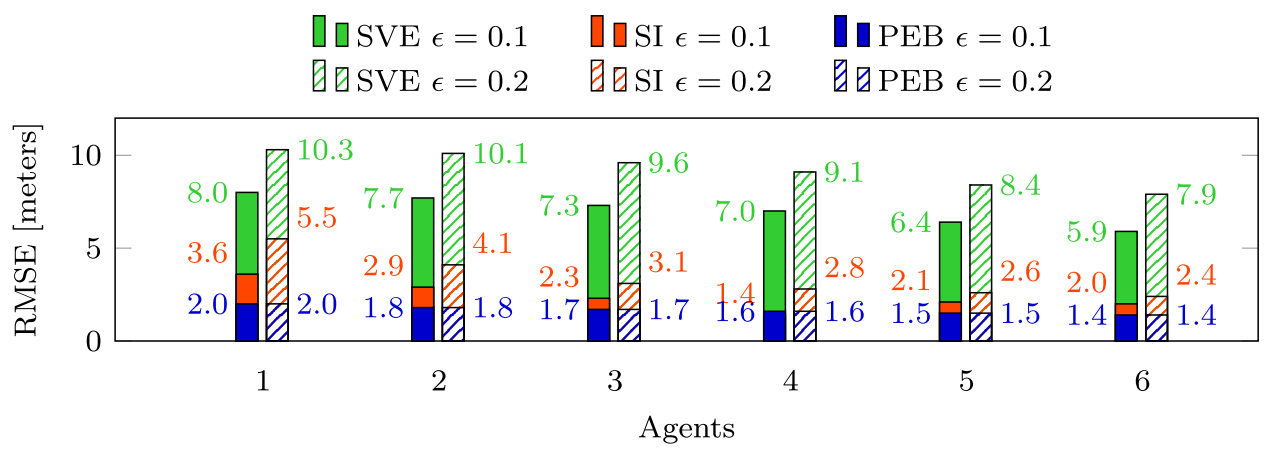

Fig. 12. Cooperative localization: localization accuracy and performance benchmark for different numbers of cooperating agents.

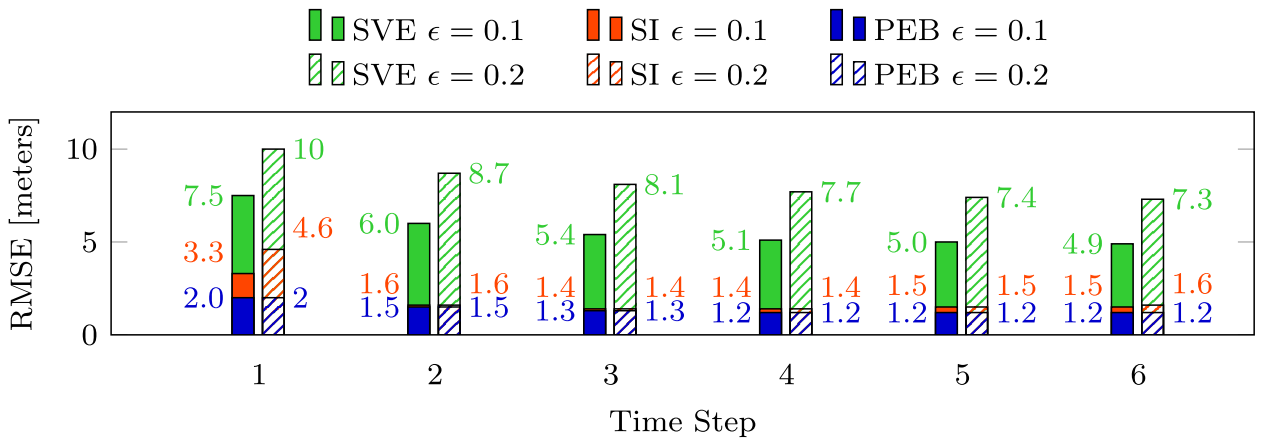

Fig. 13. Non-cooperative navigation: navigation accuracy and performance benchmark for different time steps.

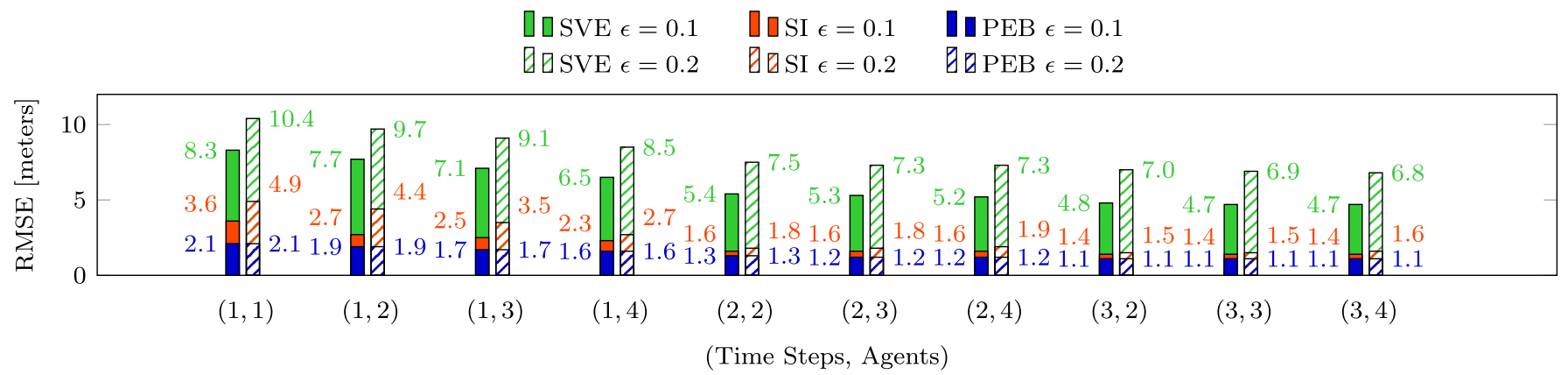

Fig. 14. Cooperative navigation: navigation accuracy and performance benchmark for different time steps and numbers of cooperating agents.

using both $\mathrm{DE}$ and $\mathrm{AE}$. Also observe that the performance of SI-based localization approaches the PEB.

Consider spatial cooperation among agents. Fig. 12 shows the localization performance based on range measurements as a function of the number of cooperating agents for obstructed propagation bias $\beta=20$ meters and different values of $\epsilon$. Notice that SRI-based localization provides significant performance improvement and robustness to NLOS detection errors compared to DE-based localization. Also observe that exploiting SRI enables the filling of most of the performance gap between the DE-based localization and PEB. Note also that SRI-based localization exploits spatial cooperation better and approaches to the PEB faster with the number of cooperating agents, compared to DE-based localization.

Now, consider navigation with temporal and spatiotemporal cooperation among agents. In such a scenario, each agent follows a circular trajectory (radius of 20 meters centered at a random position) at a speed of 0.625 meters/second. The dynamic model for position inference is

$$
\Phi_{\boldsymbol{\mu}}\left(\boldsymbol{p}^{(n)}, \boldsymbol{p}^{(n-1)}\right)=\varphi\left(\boldsymbol{p}^{(n)}-\boldsymbol{p}^{(n-1)} ; \mathbf{0}, \sigma_{\mathrm{d}}^{2} \boldsymbol{I}\right)
$$

where $\sigma_{\mathrm{d}}=0.6$ meters and the localization update rate is $1 /\left(t_{n}-t_{n-1}\right)=1 \mathrm{~Hz}$ for all $n$. First, consider temporal cooperation only. Fig. 13 shows the localization performance based on range measurements as a function of the time step for obstructed propagation bias $\beta=20$ meters and different values of $\epsilon$. Notice that SRI-based navigation with temporal cooperation provides significant performance improvement and robustness to NLOS detection errors compared to DEbased navigation. Also observe that exploiting SRI enables the filling of most of the performance gap between DE-based navigation and PEB. We now quantify the benefits due to spatial, in addition to temporal, cooperation. Fig. 14 shows the localization performance as a function of the time step and the number of cooperating agents in the same scenario considered in Fig. 13. Notice that SRI-based navigation exploits 


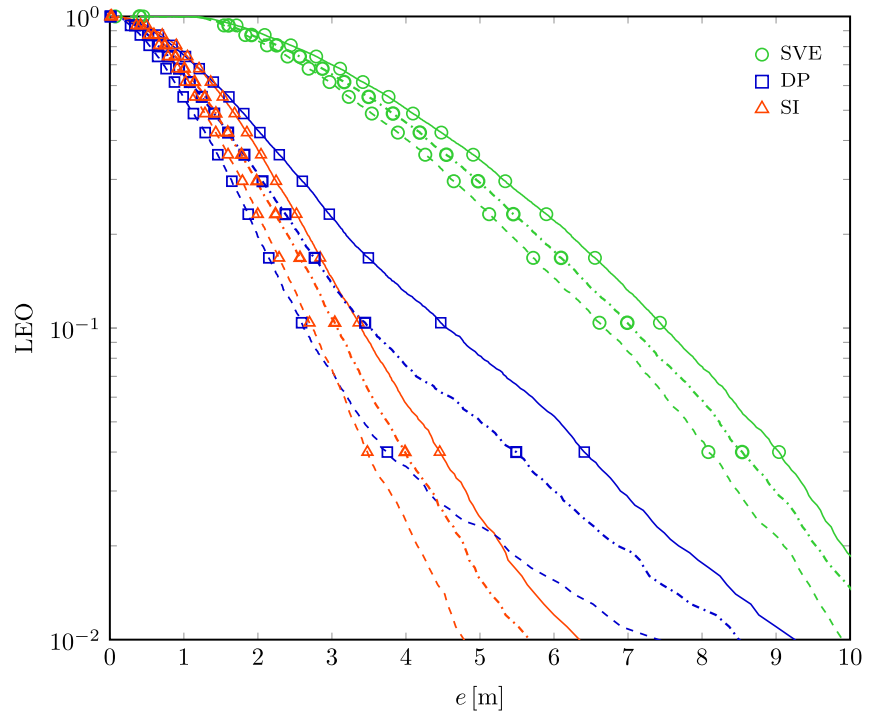

(a) IEEE 802.15.4 a indoor residential

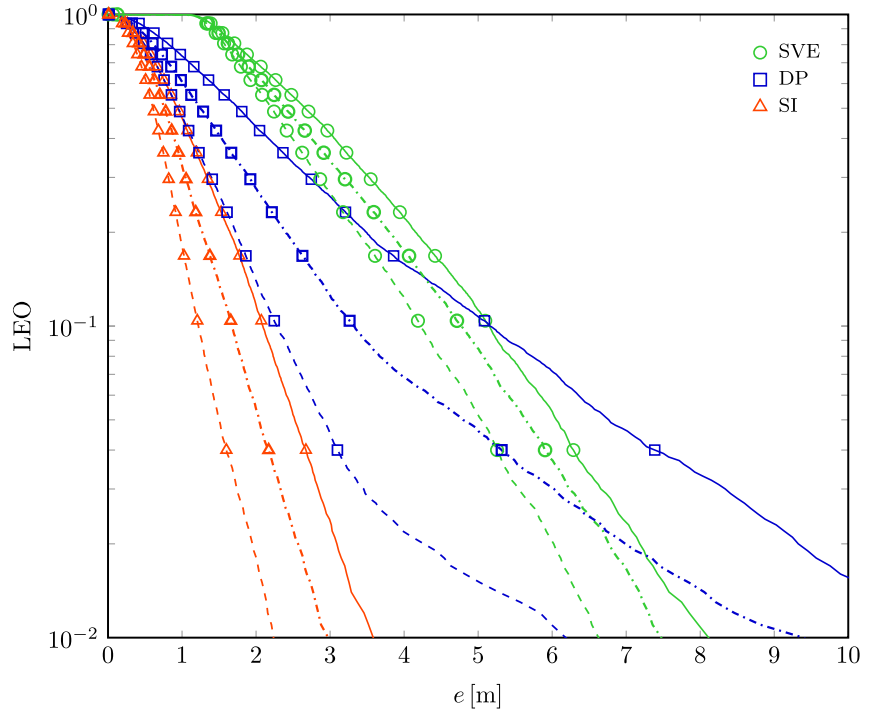

(b) IEEE 802.15.4 a outdoor

Fig. 15. LEO in two IEEE 802.15 .4 a channels with different values of NLOS probability $p_{\mathrm{NLOS}}=0.2$ (dashed), 0.5 (dashdotted), and 0.8 (solid). The performance of SVE-based localization (green circle), DP (blue square), and SI-based localization (red triangle) is shown.

spatiotemporal cooperation better than DE-based localization by accentuating the performance improvement and robustness to NLOS conditions. Moreover, SRI-based localization with spatiotemporal cooperation approaches to the PEB faster with the number of cooperating agents, compared to DE-based localization.

\section{B. CS-II: Measurements Based on IEEE 802.15.4 a Standard}

Consider a network in a 20 meters by 20 meters area with four anchors located at the corners of the square and agents randomly deployed therein. This case study compares the performance of SVE-based localization, DP, and SI-based localization in terms of localization error outage (LEO) defined as the empirical probability that the localization error is above a target value. The anchors emit UWB root raised cosine pulses (roll-off factor 0.6 and pulse width parameter $0.95 \mathrm{~ns}$ ) in the European lower band $[3.1,4.8] \mathrm{GHz}$ with maximum power spectral density $-42 \mathrm{dBm} / \mathrm{MHz}$. The emitted pulses propagate through a multipath channel modeled according to the IEEE 802.15.4 a standard for indoor residential environments with probability $p_{\text {NLOS }}$ of being in NLOS conditions. The signal-to-noise ratio at $1 \mathrm{~m}$ from the transmitter is $30 \mathrm{~dB}$. SVE-based technique uses DE from each anchor, which is obtained from the delay $\tau_{\max }$ corresponding to the maximum correlation value between the received waveform and the transmitted pulse. DP technique processes the received waveform according to the algorithm proposed in [141] with covariance matrices estimated from received waveform samples during the off-line phase. SI-based technique employs a 3modal Gaussian generative model and exploits dimensionality reduction by considering $\psi(\boldsymbol{y})$ as a vector of four elements including $\tau_{\max }$, the maximum value of the correlation, and two principal components obtained from PCA as in [55]. We compare SVE-based, DP, and SI-based techniques for inferring agent positions based on the MMSE criterion using the same measurements.

Fig. 15 shows the LEO based on received waveform measurements generated according to IEEE 802.15.4 a standard for the indoor residential and the outdoor channel models with $p_{\mathrm{NLOS}}=0.2,0.5$, and 0.8. Notice that SI-based localization exploiting SRI provides significant LEO improvement as well as robustness to NLOS propagation conditions compared to DP and SVE-based localization. For example, in indoor residential channel with $p_{\mathrm{NLOS}}=0.2$ the localization error is above $4 \mathrm{~m}$ in about $40 \%$ of cases for DE-based localization, in about $4 \%$ of cases for DP, and in about $2 \%$ of the cases for SI-based localization. In more severe NLOS propagation conditions with $p_{\mathrm{NLOS}}=0.8$, the localization error is above $4 \mathrm{~m}$ in about $50 \%$ of cases for DE-based localization, in about $13 \%$ of cases for DP, and only in about $6 \%$ of the cases for the SI-based localization. In the outdoor channel with $p_{\mathrm{NLOS}}=0.2$, the localization error is above $3 \mathrm{~m}$ in about $27 \%$ of cases for DE-based localization, in about $4 \%$ of cases for DP, and in about $2 \%$ of the cases for SI-based localization. With $p_{\mathrm{NLOS}}=0.8$, the localization error is above $3 \mathrm{~m}$ in about $41 \%$ of cases for DE-based localization, in about $26 \%$ of cases for DP, and only in about $2 \%$ of the cases for the SI-based localization. This shows that, also in IEEE 802.15.4 a standard scenario, SI-based localization is superior to DP and SVEbased localization, especially in harsh propagation conditions.

\section{FINAL REMARK}

This paper introduced the concept of LoT and proposed a new approach for accurate inference of positional states. The proposed approach exploits SI that combines SFI and SCI extracted from measurements and contextual data, respectively. We described efficient techniques for learning and exploiting the SI based on reduced data sets. Various case studies are presented for different wireless environments. In particular, 
the localization performance is quantified for sensing measurements based on noisy features and NLOS detection, and IEEE 802.15.4a standard. Results show that SI-based localization significantly outperforms DP and SVE-based localization, especially in harsh propagation conditions. Indeed, SI-based techniques are vital for LoT, especially when devices are designed for communication rather than for localization. Furthermore, the exploitation of SI offers robustness to wireless propagation conditions, thereby opening the way to a new level of accuracy for the LoT.

\section{ACKNOWLEDGMENT}

The authors wish to thank R. Cohen, W. Dai, Z. Liu, A. A. Saucan, Y. Shen, and Z. Yu for their helpful suggestions.

\section{REFERENCES}

[1] M. Z. Win, A. Conti, S. Mazuelas, Y. Shen, W. M. Gifford, D. Dardari, and M. Chiani, "Network localization and navigation via cooperation," IEEE Commun. Mag., vol. 49, no. 5, pp. 56-62, May 2011.

[2] K. Pahlavan, X. Li, and J.-P. Mäkelä, "Indoor geolocation science and technology," IEEE Commun. Mag., vol. 40, no. 2, pp. 112-118, Feb. 2002.

[3] N. Patwari, J. N. Ash, S. Kyperountas, A. O. Hero, R. L. Moses, and N. S. Correal, "Locating the nodes: Cooperative localization in wireless sensor networks," IEEE Signal Process. Mag., vol. 22, no. 4, pp. 54-69, Jul. 2005.

[4] A. H. Sayed, A. Tarighat, and N. Khajehnouri, "Network-based wireless location: Challenges faced in developing techniques for accurate wireless location information," IEEE Signal Process. Mag., vol. 22, no. 4, pp. 24-40, Jul. 2005.

[5] M. Z. Win, F. Meyer, Z. Liu, W. Dai, S. Bartoletti, and A. Conti, "Efficient multi-sensor localization for the Internet-of-Things," IEEE Signal Process. Mag., vol. 35, no. 5, pp. 153-167, Sep. 2018.

[6] J. Thomas, J. Welde, G. Loianno, K. Daniilidis, and V. Kumar, "Autonomous flight for detection, localization, and tracking of moving targets with a small quadrotor," IEEE Robot. Autom. Lett., vol. 2, no. 3, pp. 1762-1769, Jul. 2017.

[7] D. Wu, D. Chatzigeorgiou, K. Youcef-Toumi, and R. Ben-Mansour, "Node localization in robotic sensor networks for pipeline inspection," IEEE Trans. Ind. Informat., vol. 12, no. 2, pp. 809-819, Apr. 2016.

[8] R. Karlsson and F. Gustafsson, "The future of automotive localization algorithms: Available, reliable, and scalable localization: Anywhere and anytime," IEEE Signal Process. Mag., vol. 34, no. 2, pp. 60-69, Mar. 2017.

[9] H. Liu, F. Sun, B. Fang, and X. Zhang, "Robotic room-level localization using multiple sets of sonar measurements," IEEE Trans. Instrum. Meas., vol. 66, no. 1, pp. 2-13, Jan. 2017.

[10] G. Zhan and W. Shi, "LOBOT: Low-cost, self-contained localization of small-sized ground robotic vehicles," IEEE Trans. Parallel Distrib. Syst., vol. 24, no. 4, pp. 744-753, Apr. 2013.

[11] S. Bartoletti, A. Conti, and M. Z. Win, "Device-free counting via wideband signals," IEEE J. Sel. Areas Commun., vol. 35, no. 5, pp. 1163-1174, May 2017.

[12] X. Ying, S. Roy, and R. Poovendran, "Pricing mechanisms for crowdsensed spatial-statistics-based radio mapping," IEEE Trans. on Cogn. Commun. Netw., vol. 3, no. 2, pp. 242-254, Jun. 2017.

[13] R. Estrada, R. Mizouni, H. Otrok, A. Ouali, and J. Bentahar, "A crowdsensing framework for allocation of time-constrained and locationbased tasks," IEEE Trans. Services Comput., vol. PP, no. 99, pp. 1-1, Jul. 2017.

[14] F. Zabini and A. Conti, "Inhomogeneous Poisson sampling of finiteenergy signals with uncertainties in $\mathbb{R}^{d}$," IEEE Trans. Signal Process., vol. 64, no. 18, pp. 4679-4694, Sep. 2016.

[15] D. Estrin, L. Girod, G. Pottie, and M. Srivastava, "Instrumenting the world with wireless sensor networks," in Proc. IEEE Int. Conf. Acoustics, Speech, and Signal Process., vol. 4, Salt Lake City, UT, May 2001, pp. 2033-2036.

[16] I. F. Akyildiz, W. Su, Y. Sankarasubramaniam, and E. Cayirci, "A survey on sensor networks," IEEE Commun. Mag., vol. 40, no. 8, pp. 102-114, Aug. 2002.
[17] D. Dardari, A. Conti, C. Buratti, and R. Verdone, "Mathematical evaluation of environmental monitoring estimation error through energyefficient wireless sensor networks," IEEE Trans. Mobile Comput., vol. 6, no. 7, pp. 790-802, Jul. 2007.

[18] N. M. Freris, H. Kowshik, and P. R. Kumar, "Fundamentals of large sensor networks: Connectivity, capacity, clocks and computation," Proc. IEEE, vol. 98, no. 1, pp. 1828-1846, Nov. 2010.

[19] L.-L. Xie and P. Kumar, "A network information theory for wireless communication: Scaling laws and optimal operation," IEEE Trans. Inf. Theory, vol. 50, no. 5, pp. 748-767, May 2004.

[20] H. Ahmadi, A. Polo, T. Moriyama, M. Salucci, and F. Viani, "Semantic wireless localization of WiFi terminals in smart buildings," AGU Radio Science, vol. 51, no. 6, pp. 876-892, Jun. 2016.

[21] K. Lin, M. Chen, J. Deng, M. M. Hassan, and G. Fortino, "Enhanced fingerprinting and trajectory prediction for IoT localization in smart buildings," IEEE Trans. Autom. Sci. Eng., vol. 13, no. 3, pp. 1294 1307, Jul. 2016.

[22] V. Moreno, M. A. Zamora, and A. F. Skarmeta, "A low-cost indoor localization system for energy sustainability in smart buildings," IEEE Sensors J., vol. 16, no. 9, pp. 3246-3262, May 2016.

[23] G. Cardone, L. Foschini, P. Bellavista, A. Corradi, C. Borcea, M. Talasila, and R. Curtmola, "Fostering participaction in smart cities: a geosocial crowdsensing platform," IEEE Commun. Mag., vol. 51, no. 6, pp. 112-119, Jun. 2013.

[24] A. Zanella, N. Bui, A. Castellani, L. Vangelista, and M. Zorzi, "Internet of things for smart cities," IEEE Internet Things J., vol. 1, no. 1, pp. 22-32, Feb. 2014.

[25] G. Pasolini, C. Buratti, L. Feltrin, F. Zabini, C. De Castro, R. Verdone, and O. Andrisano, "Smart city pilot projects using LoRa and IEEE 802.15.4 technologies," Sensors, vol. 18, no. 4, 2018.

[26] D. Balakrishnan and A. Nayak, "An efficient approach for mobile asset tracking using contexts," IEEE Trans. Parallel Distrib. Syst., vol. 23, no. 2, pp. 211-218, Feb. 2012.

[27] E. Paolini, A. Giorgetti, M. Chiani, R. Minutolo, and M. Montanari, "Localization capability of cooperative anti-intruder radar systems," EURASIP J. Adv. Signal Process., vol. 2008, Apr. 2008.

[28] K. Witrisal, P. Meissner, E. Leitinger, Y. Shen, C. Gustafson, F. Tufvesson, K. Haneda, D. Dardari, A. F. Molisch, A. Conti, and M. Z. Win, "High-accuracy localization for assisted living," IEEE Signal Process. Mag., vol. 33, no. 2, pp. 59-70, Mar. 2016.

[29] M. Driusso, C. Marshall, M. Sabathy, F. Knutti, H. Mathis, and F. Babich, "Vehicular position tracking using LTE signals," IEEE Trans. Veh. Technol., vol. 66, no. 4, pp. 3376-3391, Apr. 2017.

[30] K. Daniilidis, C. Krauss, M. Hansen, and G. Sommer, "Real-time tracking of moving objects with an active camera," Real-Time Imaging, vol. 4, no. 1, pp. 3-20, Feb. 1998.

[31] S. D'Oro, L. Galluccio, G. Morabito, and S. Palazzo, "Exploiting object group localization in the Internet of things: Performance analysis," IEEE Trans. Veh. Technol., vol. 64, no. 8, pp. 3645-3656, Aug 2015.

[32] L. Chen, S. Thombre, K. Järvinen, E. S. Lohan, A. Alén-Savikko, H. Leppäkoski, M. Z. H. Bhuiyan, S. Bu-Pasha, G. N. Ferrara, S. Honkala, J. Lindqvist, L. Ruotsalainen, P. Korpisaari, and H. Kuusniemi, "Robustness, security and privacy in location-based services for future IoT: A survey," IEEE Access, vol. 5, pp. 8956-8977, Apr. 2017.

[33] D. Zhang, L. T. Yang, M. Chen, S. Zhao, M. Guo, and Y. Zhang, "Real-time locating systems using active RFID for Internet of Things," IEEE Syst. J., vol. 10, no. 3, pp. 1226-1235, Sep. 2016.

[34] D. Zhang, S. Zhao, L. T. Yang, M. Chen, Y. Wang, and H. Liu, "NextMe: Localization using cellular traces in Internet of things," IEEE Trans. Ind. Informat., vol. 11, no. 2, pp. 302-312, Apr. 2015.

[35] F. Meyer, B. Etzlinger, Z. Liu, F. Hlawatsch, and M. Z. Win, "A scalable algorithm for network localization and synchronization," IEEE Internet Things J., vol. 5, no. 6, pp. 4714-4727, Dec. 2018, special issue on Towards Positioning, Navigation, and Location Based Services (PNLBS) for Internet of Things.

[36] S. G. Nagarajan, P. Zhang, and I. Nevat, "Geo-spatial location estimation for Internet of things (IoT) networks with one-way time-of-arrival via stochastic censoring," IEEE Internet Things J., vol. 4, no. 1, pp. 205-214, Feb. 2017.

[37] D. B. Jourdan, D. Dardari, and M. Z. Win, "Position error bound for UWB localization in dense cluttered environments," IEEE Trans. Aerosp. Electron. Syst., vol. 44, no. 2, pp. 613-628, Apr. 2008.

[38] Y. Shen and M. Z. Win, "Fundamental limits of wideband localization Part I: A general framework," IEEE Trans. Inf. Theory, vol. 56, no. 10, pp. 4956-4980, Oct. 2010. 
[39] Y. Shen, H. Wymeersch, and M. Z. Win, "Fundamental limits of wideband localization - Part II: Cooperative networks," IEEE Trans. Inf. Theory, vol. 56, no. 10, pp. 4981-5000, Oct. 2010.

[40] Y. Shen, S. Mazuelas, and M. Z. Win, "Network navigation: Theory and interpretation," IEEE J. Sel. Areas Commun., vol. 30, no. 9, pp. 1823-1834, Oct. 2012.

[41] M. Z. Win, Y. Shen, and W. Dai, "A theoretical foundation of network localization and navigation," Proc. IEEE, vol. 106, no. 7, pp. 1136-1165, Jul. 2018, special issue on Foundations and Trends in Localization Technologies.

[42] M. J. D. Rendas and J. M. F. Moura, "Cramér-Rao bound for location systems in multipath environments," IEEE Trans. Signal Process., vol. 39, no. 12, pp. 2593-2610, Dec. 1991

[43] C. Chang and A. Sahai, "Estimation bounds for localization," in Proc. IEEE Conf. on Sensor and Ad Hoc Commun. and Networks, S. Clara, CA, USA, Oct. 2004, pp. 415-424.

[44] L. Mailaender, "On the geolocation bounds for round-trip time-ofarrival and all non-line-of-sight channels," EURASIP J. Adv. in Signal Process., vol. 2008, pp. 1-10, 2008.

[45] Y. Qi, H. Kobayashi, and H. Suda, "Analysis of wireless geolocation in a non-line-of-sight environment," IEEE Trans. Wireless Commun., vol. 5, no. 3, pp. 672-681, Mar. 2006.

[46] S. Gezici, H. Celebi, H. V. Poor, and H. Arslan, "Fundamental limits on time delay estimation in dispersed spectrum cognitive radio systems," IEEE Trans. Wireless Commun., vol. 8, no. 1, pp. 78-83, Jan. 2009.

[47] H. Wymeersch, J. Lien, and M. Z. Win, "Cooperative localization in wireless networks," Proc. IEEE, vol. 97, no. 2, pp. 427-450, Feb. 2009, special issue on Ultra-Wide Bandwidth (UWB) Technology \& Emerging Applications.

[48] T. V. Nguyen, Y. Jeong, H. Shin, and M. Z. Win, "Least-square cooperative localization," IEEE Trans. Veh. Technol., vol. 64, no. 4, pp. 1318-1330, Apr. 2015.

[49] A. T. Ihler, J. W. Fisher III, R. L. Moses, and A. S. Willsky, "Nonparametric belief propagation for self-localization of sensor networks," IEEE J. Sel. Areas Commun., vol. 23, no. 4, pp. 809-819, Apr. 2005.

[50] A. Giorgetti and M. Chiani, "Time-of-arrival estimation based on information theoretic criteria," IEEE Trans. Signal Process., vol. 61, pp. 1869-1879, Apr. 2013.

[51] S. Bartoletti, W. Dai, A. Conti, and M. Z. Win, "A mathematical model for wideband ranging," IEEE J. Sel. Topics Signal Process., vol. 9, no. 2, pp. 216-228, Mar. 2015.

[52] S. Bartoletti, A. Conti, A. Giorgetti, and M. Z. Win, "Sensor radar networks for indoor tracking," IEEE Wireless Commun. Lett., vol. 3, no. 2, pp. 157-160, Apr. 2014.

[53] S. Mazuelas, Y. Shen, and M. Z. Win, "Belief condensation filtering," IEEE Trans. Signal Process., vol. 61, no. 18, pp. 4403-4415, Sep. 2013.

[54] F. Meyer, T. Kropfreiter, J. L. Williams, R. A. Lau, F. Hlawatsch, P. Braca, and M. Z. Win, "Message passing algorithms for scalable multitarget tracking," Proc. IEEE, vol. 106, no. 2, pp. 221-259, Feb. 2018.

[55] S. Mazuelas, A. Conti, J. C. Allen, and M. Z. Win, "Soft range information for network localization," IEEE Trans. Signal Process., vol. 66, no. 12, pp. 3155-3168, Jun. 2018.

[56] H. Luecken, C. Steiner, and A. Wittneben, "Location-aware UWB communication with generalized energy detection receivers," IEEE Trans. Wireless Commun., vol. 11, no. 9, pp. 3068-3078, Sep. 2012.

[57] S. Bartoletti, A. Conti, W. Dai, and M. Z. Win, "Threshold profiling for wideband ranging," IEEE Signal Process. Lett., vol. 25, no. 6, pp. 873-877, Jun. 2018.

[58] T. Sathyan and M. Hedley, "Fast and accurate cooperative tracking in wireless networks," IEEE Trans. Mobile Comput., vol. 12, no. 9, pp. 1801-1813, Sep. 2013.

[59] J. Hightower and G. Borriello, "Location systems for ubiquitous computing," Computer, vol. 34, no. 8, pp. 57-66, Aug. 2001.

[60] U. A. Khan, S. Kar, and J. M. F. Moura, "Distributed sensor localization in random environments using minimal number of anchor nodes," IEEE Trans. Signal Process., vol. 57, no. 5, pp. 2000-2016, May 2009.

[61] U. A. Khan, S. Kar, and J. M. F. Moura, "DILAND: An algorithm for distributed sensor localization with noisy distance measurements," IEEE Trans. Signal Process., vol. 58, no. 3, pp. 1940-1947, Mar. 2010.

[62] S. Kar, B. Sinopoli, and J. M. F. Moura, "Kalman filtering with intermittent observations: Weak convergence to a stationary distribution," IEEE Trans. Autom. Control, vol. 57, no. 2, pp. 405-420, Feb. 2012.

[63] U. A. Khan and J. M. F. Moura, "Distributing the Kalman filter for large-scale systems," IEEE Trans. Signal Process., vol. 56, no. 10, pp. 4919-4935, Oct. 2008.
[64] M. Çetin, L. Chen, J. W. Fisher III, A. T. Ihler, R. L. Moses, M. J. Wainwright, and A. S. Willsky, "Distributed fusion in sensor networks," IEEE Signal Process. Mag., vol. 23, no. 4, pp. 42-55, Jul 2006.

[65] G. Mao, B. Fidan, and B. D. O. Anderson, "Wireless sensor network localization techniques," Comput. Networks, vol. 51, no. 10, pp. 25292553, 2007.

[66] J. H. Kotecha and P. M. Djuric, "Gaussian sum particle filtering," IEEE Trans. Signal Process., vol. 51, no. 10, pp. 2602-1612, Oct. 2003.

[67] P. Djuric, J. Kotecha, J. Zhang, Y. Huang, T. Ghirmai, M. Bugallo, and J. Miguez, "Particle filtering," IEEE Signal Process. Mag., vol. 20, no. 5, pp. 19-38, 2003.

[68] D. Dardari, P. Closas, and P. M. Djurić, "Indoor tracking: Theory, methods, and technologies," IEEE Trans. Veh. Technol., vol. 64, no. 4, pp. 1263-1278, Apr. 2015.

[69] T. L. T. Nguyen, F. Septier, H. Rajaona, G. W. Peters, I. Nevat, and Y. Delignon, "A Bayesian perspective on multiple source localization in wireless sensor networks," IEEE Trans. Signal Process., vol. 64, no. 7, pp. 1684-1699, Apr. 2016.

[70] R. Niu, A. Vempaty, and P. K. Varshney, "Received signal strength based localization in wireless sensor networks," Proc. IEEE, vol. 106, no. 6, pp. 1166 - 1182, Jun. 2018.

[71] S. Safavi, U. A. Khan, S. Kar, and J. M. F. Moura, "Distributed localization: A linear theory," Proc. IEEE, vol. 106, no. 7, pp. 1204 1223, Jul. 2018.

[72] M. Chiani, A. Giorgetti, and E. Paolini, "Sensor radar for object tracking," Proc. IEEE, vol. 106, no. 6, pp. 1022-1041, Jun. 2018.

[73] S. Aditya, A. F. Molisch, and H. M. Behairy, "A survey on the impact of multipath on wideband time-of-arrival based localization," Proc. IEEE, vol. 106, no. 7, pp. 1183-1203, Jul. 2018.

[74] R. M. Buehrer, H. Wymeersch, and R. M. Vaghefi, "Collaborative sensor network localization: Algorithms and practical issues," Proc. IEEE, vol. 106, no. 6, pp. 1089-1114, Jun. 2018.

[75] J. Ureña, Á. Hernández, J. J. García, J. M. Villadangos, M. C. Pérez, D. Gualda, F. J. Álvarez, and T. Aguilera, "Acoustic local positioning with encoded emission beacons," Proc. IEEE, vol. 106, no. 6, pp. 10421062, Jun. 2018.

[76] M. F. Keskin, A. D. Sezer, and S. Gezici, "Localization via visible light systems," Proc. IEEE, vol. 106, no. 6, pp. 1063-1088, Jun. 2018.

[77] C. Morelli, M. Nicoli, V. Rampa, and U. Spagnolini, "Hidden Markov models for radio localization in mixed LOS/NLOS conditions," IEEE Trans. Signal Process., vol. 55, no. 4, pp. 1525-1542, Apr 2007.

[78] I. Dokmanić, R. Parhizkar, J. Ranieri, and M. Vetterli, "Euclidean distance matrices: Essential theory, algorithms, and applications," IEEE Signal Process. Mag., vol. 32, no. 6, pp. 12-30, Nov. 2015.

[79] M. Rydstrom, L. Reggiani, E. Strom, and A. Svensson, "Suboptimal soft range estimators with applications in UWB sensor networks," IEEE Trans. Signal Process., vol. 56, no. 10, pp. 4856-4866, Oct. 2008.

[80] W. Dai, Y. Shen, and M. Z. Win, "Distributed power allocation for cooperative wireless network localization," IEEE J. Sel. Areas Commun., vol. 33, no. 1, pp. 28-40, Jan. 2015.

[81] J. Chen, W. Dai, Y. Shen, V. K. Lau, and M. Z. Win, "Power management for cooperative localization: A game theoretical approach," IEEE Trans. Signal Process., vol. 64, no. 24, pp. 6517-6532, Dec. 2016.

[82] T. Wang, G. Leus, and L. Huang, "Ranging energy optimization for robust sensor positioning based on semidefinite programming," IEEE Trans. Signal Process., vol. 57, no. 12, pp. 4777-4787, Dec. 2009.

[83] H. Godrich, A. P. Petropulu, and H. V. Poor, "Power allocation strategies for target localization in distributed multiple-radar architectures," IEEE Trans. Signal Process., vol. 59, no. 7, pp. 3226-3240, Jul. 2011.

[84] X. Shen and P. K. Varshney, "Sensor selection based on generalized information gain for target tracking in large sensor networks," IEEE Trans. Signal Process., vol. 62, no. 2, pp. 363-375, Jan. 2014.

[85] T. Wang, Y. Shen, A. Conti, and M. Z. Win, "Network navigation with scheduling: Error evolution," IEEE Trans. Inf. Theory, vol. 63, no. 11, pp. 7509-7534, Nov. 2017.

[86] S. Dwivedi, D. Zachariah, A. De Angelis, and P. Händel, "Cooperative decentralized localization using scheduled wireless transmissions," IEEE Commun. Lett., vol. 17, no. 6, pp. 1240-1243, Jun. 2013.

[87] L. Shi, M. Epstein, B. Sinopoli, and R. M. Murray, "Effective sensor scheduling schemes in a sensor network by employing feedback in the communication loop," in Proc. IEEE Int. Conf. Control Applications, Singapore, Oct. 2007, pp. 1006-1011.

[88] W. Dai, Y. Shen, and M. Z. Win, "A computational geometry framework for efficient network localization," IEEE Trans. Inf. Theory, vol. 64, no. 2, pp. 1317-1339, Feb. 2018. 
[89] S. Mazuelas, R. M. Lorenzo, A. Bahillo, P. Fernandez, J. Prieto, and E. J. Abril, "Topology assessment provided by weighted barycentric parameters in harsh environment wireless location systems," IEEE Trans. Signal Process., vol. 58, no. 7, pp. 3842-3857, Jul. 2010.

[90] M. Z. Win, W. Dai, Y. Shen, G. Chrisikos, and H. V. Poor, "Network operation strategies for efficient localization and navigation," Proc. IEEE, vol. 106, no. 7, pp. 1224-1254, Jul. 2018, special issue on Foundations and Trends in Localization Technologies.

[91] M. Z. Win, W. Dai, and Y. Shen, "Variable resource allocation for localization," U.S. Patent 10,228,448, Mar. 12, 2019.

[92] A. Conti, M. Guerra, D. Dardari, N. Decarli, and M. Z. Win, "Network experimentation for cooperative localization," IEEE J. Sel. Areas Commun., vol. 30, no. 2, pp. 467-475, Feb. 2012.

[93] A. Conti, D. Dardari, M. Guerra, L. Mucchi, and M. Z. Win, "Experimental characterization of diversity navigation," IEEE Syst. J., vol. 8, no. 1, pp. 115-124, Mar. 2014.

[94] D. Fortin-Simard, J. S. Bilodeau, K. Bouchard, S. Gaboury, B. Bouchard, and A. Bouzouane, "Exploiting passive RFID technology for activity recognition in smart homes," IEEE Intell. Syst., vol. 30, no. 4, pp. 7-15, Jul. 2015

[95] Z. Liu, W. Dai, and M. Z. Win, "Mercury: An infrastructure-free system for network localization and navigation," IEEE Trans. Mobile Comput., vol. 17, no. 5, pp. 1119-1133, May 2018

[96] B. Denis, R. Raulefs, B. H. Fleury, B. Uguen, N. Amiot, L. de Celis, J. Dominguez, M. B. Koldsgaard, M. Laaraiedh, H. Noureddine, E. Staudinger, and G. Steinboeck, "Cooperative and heterogeneous indoor localization experiments," in Proc. IEEE Int. Conf. Commun. Workshop, Jun. 2013, pp. 6-10, budapest, Hungary.

[97] S. Mazuelas, F. A. Lago, J. Blas, A. Bahillo, P. Fernandez, R. Lorenzo, and E. Abril, "Prior NLOS measurements correction for positioning in cellular wireless networks," IEEE Trans. Vehicular Technology, vol. 58, no. 5, pp. 2585-2591, Jun 2009.

[98] S. Maranò, W. M. Gifford, H. Wymeersch, and M. Z. Win, "NLOS identification and mitigation for localization based on UWB experimental data," IEEE J. Sel. Areas Commun., vol. 28, no. 7, pp. 1026-1035, Sep. 2010.

[99] I. Güvenç, C.-C. Chong, F. Watanabe, and H. Inamura, "NLOS identification and weighted least-squares localization for UWB systems using multipath channel statistics," EURASIP J. Adv. in Signal Process. vol. 2008, pp. 1-14, Article ID 271 984, 2008.

[100] J. Khodjaev, Y. Park, and A. S. Malik, "Survey on NLOS identification and error mitigation problems in UWB-based positioning algorithms for dense environments," in Annals of Telecommunications, 2009.

[101] H. Wymeersch, S. Maranò, W. M. Gifford, and M. Z. Win, "A machine learning approach to ranging error mitigation for UWB localization," IEEE Trans. Commun., vol. 60, no. 6, pp. 1719-1728, Jun. 2012.

[102] J.-Y. Lee and R. A. Scholtz, "Ranging in a dense multipath environment using an UWB radio link," IEEE J. Sel. Areas Commun., vol. 20, no. 9, pp. 1677-1683, Dec. 2002.

[103] J. Schroeder, S. Galler, K. Kyamakya, and T. Kaiser, "Threedimensional indoor localization in non line of sight UWB channels," in Proc. IEEE Int. Conf. on Ultra-Wideband, Singapore, Sep. 2007.

[104] R. Casas, A. Marco, J. J. Guerrero, and J. Falcó, "Robust estimator for non-line-of-sight error mitigation in indoor localization," EURASIP J. Appl. Signal Process., no. 1, pp. 156-156, 2006.

[105] S. Venkatesh and R. M. Buehrer, "NLOS mitigation using linear programming in ultrawideband location-aware networks," IEEE Trans. Veh. Technol., vol. 56, no. 5, pp. 3182-3198, Sep. 2007.

[106] J. Schroeder, S. Galler, K. Kyamakya, and K. Jobmann, "NLOS detection algorithms for ultra-wideband localization," in Workshop on Positioning, Navigation and Commun. (WPNC), Hannover, Germany, Mar. 2007, pp. 159-166.

[107] B. Denis and N. Daniele, "NLOS ranging error mitigation in a distributed positioning algorithm for indoor UWB ad-hoc networks," in International Workshop on Wireless Ad-Hoc Networks, Oulu, Finland, May/Jun. 2004, pp. 356-360.

[108] B. Denis, M. Maman, and L. Ouvry, "On the scheduling of ranging and distributed positioning updates in cooperative IR-UWB networks," in Proc. IEEE Int. Conf. on Ultra-Wideband, Vancouver, BC, Sep. 2009, pp. 370-375.

[109] B. Denis, J.Keignart, and N. Daniele, "Impact of NLOS propagation upon ranging precision in UWB systems," in Proc. IEEE Conf. on Ultra Wideband Syst. and Technol., Nov. 2003, pp. 379-383.

[110] B. Cakmak, D. N. Urup, F. Meyer, T. Pedersen, B. H. Fleury, and F. Hlawatsch, "Cooperative localization for mobile networks: A distributed belief propagation-mean field message passing algorithm," IEEE Signal Process. Lett., vol. 23, pp. 828-832, 2016.
[111] A. Haghparast, T. Abrudan, and V. Koivunen, "OFDM ranging in multipath channels using time reversal method," in Proc. IEEE Workshop on Signal Process. Advances in Wireless Commun., Perugia, Jun. 2009, pp. $568-572$.

[112] S. Gezici and H. V. Poor, "Position estimation via ultra-wide-band signals," Proc. IEEE, vol. 97, no. 2, pp. 386-403, Feb. 2009.

[113] S. Gezici, Z. Tian, G. B. Giannakis, H. Kobayashi, A. F. Molisch, H. V. Poor, and Z. Sahinoglu, "Localization via ultra-wideband radios: A look at positioning aspects for future sensor networks," IEEE Signal Process. Mag., vol. 22, no. 4, pp. 70-84, Jul. 2005.

[114] S. Mazuelas, A. Bahillo, R. Lorenzo, P. Fernandez, F. A. Lago, E. Garcia, J. Blas, and E. Abril, "Robust indoor positioning provided by real-time RSSI values in unmodified WLAN networks," IEEE $J$. Sel. Topics Signal Process., vol. 3, no. 5, pp. 821-831, Oct. 2009.

[115] D. Dardari, A. Conti, U. J. Ferner, A. Giorgetti, and M. Z. Win, "Ranging with ultrawide bandwidth signals in multipath environments," Proc. IEEE, vol. 97, no. 2, pp. 404-426, Feb. 2009, special issue on Ultra-Wide Bandwidth (UWB) Technology \& Emerging Applications.

[116] A. J. Weiss and E. Weinstein, "Fundamental limitations in passive time delay estimation - part I: Narrow-band systems," IEEE Trans. Acoust. Speech, Signal Process., vol. ASP-31, no. 2, pp. 472-486, Apr. 1983.

[117] E. Weinstein and A. J. Weiss, "Fundamental limitations in passive time delay estimation - part II: Wide-band systems," IEEE Trans. Acoust., Speech, Signal Process., vol. ASSP-32, no. 5, pp. 1064-1078, Oct. 1984.

[118] E. Weinstein and A. J. Weiss, "A general class of lower bounds in parameter estimation," IEEE Trans. Inf. Theory, vol. 34, no. 2, pp. 338-342, Mar. 1988

[119] J. P. Iannello, "Large and small error performance limits for multipath time delay estimation," IEEE Trans. Acoust., Speech, Signal Process. vol. ASSP-34, no. 2, pp. 245-251, Apr. 1986.

[120] A. Zeira and P. M. Schultheiss, "Realizable lower bounds for time delay estimation," IEEE Trans. Signal Process., vol. 41, no. 1, pp. 3102-3113, Nov. 1993.

[121] A. Zeira and P. M. Schultheiss, "Realizable lower bounds for time delay estimation: Part 2-threshold phenomena," IEEE Trans. Signal Process., vol. 32, no. 5, pp. 1001-1007, May 1994.

[122] J. Ziv and M. Zakai, "Some lower bounds on signal parameter estimation," IEEE Trans. Inf. Theory, vol. 15, no. 3, pp. 386-391, May 1969.

[123] S. Bellini and G. Tartara, "Bounds on error in signal parameter estimation," IEEE Trans. Commun., vol. 22, no. 3, pp. 340-342, Mar. 1974.

[124] D. Chazan, M. Zakai, and J. Ziv, "Improved lower bounds on signal parameter estimation," IEEE Trans. Inf. Theory, vol. 21, no. 1, pp. 90-93, Jan. 1975.

[125] K. L. Bell, Y. Steinberg, Y. Ephraim, and H. L. V. Trees, "Extended Ziv-Zakai lower bound for vector parameter estimation," IEEE Trans. Inf. Theory, vol. 43, no. 2, pp. 624-637, Feb. 1997.

[126] H. Anouar, A. M. Hayar, R. Knopp, and C. Bonnet, "Ziv-Zakai lower bound on the time delay estimation of UWB signals," in in Proc. Int Symposium on Commun., Control, and Signal Processing (ISCCSP), Marrakech, Morocco, Mar. 2006.

[127] B. M. Sadler, L. Huang, and Z. Xu, "Ziv-Zakai time delay estimation bound for ultra-wideband signals," in IEEE International Conference on Acoustics, Speech and Signal Processing, 2007. ICASSP 2007, vol. 3, Honolulu, HI, Apr. 2007, pp. 549-552.

[128] S. Maranò, W. M. Gifford, H. Wymeersch, and M. Z. Win, "Method and system for identification and mitigation of errors in non-line-ofsight distance estimation," U.S. Patent 9,002,286, Apr. 7, 2015.

[129] S. Maranò, W. M. Gifford, H. Wymeersch, and M. Z. Win, "Method and system for identification and mitigation of errors in non-line-ofsight distance estimation," U.S. Patent 9,602,972, Mar. 21, 2017.

[130] S. Bartoletti, A. Giorgetti, M. Z. Win, and A. Conti, "Blind selection of representative observations for sensor radar networks," IEEE Trans. Veh. Technol., vol. 64, no. 4, pp. 1388-1400, Apr. 2015.

[131] H. Godrich, A. Petropulu, and H. Poor, "Sensor selection in distributed multiple-radar architectures for localization: A knapsack problem formulation," IEEE Trans. Signal Process., vol. 60, no. 1, pp. 247-260, Jan. 2012.

[132] L. Kaplan, "Global node selection for localization in a distributed sensor network," IEEE Trans. Aerosp. Electron. Syst., vol. 42, no. 1 , pp. 113-135, Jan. 2006

[133] L. Kaplan, "Local node selection for localization in a distributed sensor network," IEEE Trans. Aerosp. Electron. Syst., vol. 42, no. 1, pp. 136146, Jan. 2006. 
[134] J. Prieto, S. Mazuelas, A. Bahillo, P. Fernandez, R. M. Lorenzo, and E. J. Abril, "Adaptive data fusion for wireless localization in harsh environments," IEEE Trans. Signal Process., vol. 60, no. 4, pp. 15851596, Apr. 2012

[135] A. Catovic and Z. Sahinoglu, "The Cramer-Rao bounds of hybrid TOA/RSS and TDOA/RSS location estimaion schemes," IEEE Commun. Lett., vol. 8, no. 10, pp. 626-628, Oct. 2004.

[136] J.-C. Chen, P. Ting, C.-S. Maa, and J.-T. Chen, "Wireless geolocation with TOA/AOA measurements using factor graph and sum-product algorithm," in Proc. IEEE Semiannual Veh. Technol. Conf., vol. 5, Sep. 2004, pp. 3526-3529.

[137] P. Deng and P. Fan, "An AOA assisted TOA positioning system," in Proc. of Int. Conf. on Commun. Technol., vol. 2, Beijing, China, 2000, pp. 1501-1504.

[138] P. Closas, C. Fernández-Prades, and J. A. Fernández-Rubio, "Direct position estimation approach outperforms conventional two-steps positioning," in Proc. of European Signal Processing Conference (EUSIPCO), Aug. 2009, pp. 1958-1962.

[139] P. Closas and A. Gusi-Amigo, "Direct position estimation of GNSS receivers: Analyzing main results, architectures, enhancements, and challenges," IEEE Signal Process. Mag., vol. 34, no. 5, pp. 72-84, Sep. 2017.

[140] L. Tzafri and A. J. Weiss, "High-resolution direct position determination using MVDR," IEEE Trans. Wireless Commun., vol. 15, no. 9, pp. 6449-6461, Sep. 2016

[141] O. Bialer, D. Raphaeli, and A. J. Weiss, "Maximum-likelihood direct position estimation in dense multipath," IEEE Trans. Veh. Technol., vol. 62, no. 5, pp. 2069-2079, Jun. 2013

[142] O. Bar-Shalom and A. J. Weiss, "Efficient direct position determination of orthogonal frequency division multiplexing signals," IET Radar, Sonar Navigation, vol. 3, no. 2, pp. 101-111, Apr. 2009

[143] N. Vankayalapati, S. Kay, and Q. Ding, "TDOA based direct positioning maximum likelihood estimator and the Cramer-Rao bound," IEEE Trans. Aerosp. Electron. Syst., vol. 50, no. 3, pp. 1616-1635, Jul. 2014.

[144] J. P. Beaudeau, M. F. Bugallo, and P. M. Djurić, "RSSI-based multitarget tracking by cooperative agents using fusion of cross-target information," IEEE Trans. Signal Process., vol. 63, no. 19, pp. 50335044, Oct. 2015

[145] M. Rydström, L. Reggiani, E. G. Ström, and A. Svensson, "Adapting the ranging algorithm to the positioning technique in UWB sensor networks," Wireless Personal Communications, vol. 47, no. 1, pp. 2738, Oct. 2008.

[146] N. Garcia, H. Wymeersch, E. G. Larsson, A. M. Haimovich, and M. Coulon, "Direct localization for massive MIMO," IEEE Trans. Signal Process., vol. 65, no. 10, pp. 2475-2487, May 2017.

[147] F. Montorsi, S. Mazuelas, G. M. Vitetta, and M. Z. Win, "On the performance limits of map-aware localization," IEEE Trans. Inf. Theory, vol. 59, no. 8, pp. 5023-5038, Aug. 2013.

[148] Y. Cheng and T. Singh, "Efficient particle filtering for road-constrained target tracking," IEEE Trans. Aerosp. Electron. Syst., vol. 43, no. 4, pp. 1454-1469, 2007.

[149] M. Anisetti, C. A. Ardagna, V. Bellandi, E. Damiani, and S. Reale, "Map-based location and tracking in multipath outdoor mobile networks," IEEE Trans. Wireless Commun., vol. 10, no. 3, pp. 814-824, 2011

[150] X. R. Li and V. P. Jilkov, "Survey of maneuvering target tracking Part I: Dynamic models," IEEE Trans. Aerosp. Electron. Syst., vol. 39, no. 4, pp. 1333-1364, Oct. 2003.

[151] E. Foxlin, "Pedestrian tracking with shoe-mounted inertial sensors," IEEE Comput. Graph. Appl., vol. 25, no. 6, pp. 38-46, Nov.-Dec. 2005.

[152] A. R. Jiménez, F. Seco, J. C. Prieto, and J. Guevara, "Accurate pedestrian indoor navigation by tightly coupling foot-mounted IMU and RFID measurements," IEEE Trans. Instrum. Meas., vol. 61, no. 1, pp. 178 - 189, Jan. 2012.

[153] J. Prieto, S. Mazuelas, and M. Z. Win, "Context-aided inertial navigation via belief condensation," IEEE Trans. Signal Process., vol. 64 no. 12, pp. 3250-3261, Jun. 2016.

[154] G. Dissanayake, S. Sukkarieh, E. Nebot, and H. Durrant-Whyte, "The aiding of a low-cost strapdown inertial measurement unit using vehicle model constraints for land vehicle applications," IEEE Trans. Robot. Autom., vol. 17, no. 5, pp. 731 - 747, Oct. 2001.

[155] I. Skog and P. Handel, "In-car positioning and navigation technologies: A survey," IEEE Trans. Intell. Transp. Syst., vol. 10, no. 1, pp. $4-21$, Mar. 2009.

[156] I. Nevat, G. W. Peters, K. Avnit, F. Septier, and L. Clavier, "Location of things: Geospatial tagging for IoT using time-of-arrival," IEEE Trans.
Signal and Inf. Process. over Networks, vol. 2, no. 2, pp. 174-185, Jun. 2016.

[157] R. Niu, R. S. Blum, P. K. Varshney, and A. L. Drozd, "Target localization and tracking in noncoherent multiple-input multiple-output radar systems," IEEE Trans. Aerosp. Electron. Syst., vol. 48, no. 2, pp. 1466-1489, Apr. 2012.

[158] R. Niu, B. Chen, and P. K. Varshney, "Fusion of decisions transmitted over Rayleigh fading channels in wireless sensor networks," IEEE Trans. Signal Process., vol. 54, no. 3, pp. 1018-1027, Mar. 2006.

[159] R. Niu and P. K. Varshney, "Target location estimation in sensor networks with quantized data," IEEE Trans. Signal Process., vol. 54, no. 12, pp. 4519-4528, Dec. 2006.

[160] R. Viswanathan and P. K. Varshney, "Distributed detection with multiple sensors - Part I : Fundamentals," Proc. IEEE, vol. 85, no. 1, pp. 54-63, Jan. 1997.

[161] Z. Chair and P. K. Varshney, "Optimal data fusion in multiple sensor detection systems," IEEE Trans. Aerosp. Electron. Syst., vol. 22, no. 1 pp. 98-101, Jan. 1986.

[162] B. Chen, L. Tong, and P. K. Varshney, "Channel-aware distributed detection in wireless sensor networks," IEEE Signal Process. Mag., vol. 23, no. 4, pp. 16-26, Jul 2006

[163] W. Du, J. Deng, Y. S. Han, S. Chen, and P. K. Varshney, "A key predistribution scheme for sensor networks using deployment knowledge," IEEE Trans. Depend. Sec. Comput., vol. 3, no. 1, pp. 62-77, Jan. 2006.

[164] E. Masazade, R. Niu, and P. K. Varshney, "Dynamic bit allocation for object tracking in wireless sensor networks," IEEE Trans. Signal Process., vol. 60, no. 10, pp. 5048-5063, Oct. 2012.

[165] Y. Shen, W. Dai, and M. Z. Win, "Power optimization for network localization," IEEE/ACM Trans. Netw., vol. 22, no. 4, pp. 1337-1350, Aug. 2014.

[166] W. Dai, Y. Shen, and M. Z. Win, "Energy-efficient network navigation algorithms," IEEE J. Sel. Areas Commun., vol. 33, no. 7, pp. 14181430, Jul. 2015

[167] J. Chen, W. Dai, Y. Shen, V. K. Lau, and M. Z. Win, "Resource management games for distributed network localization," IEEE J. Sel. Areas Commun., vol. 35, no. 2, pp. 317-329, Feb. 2017.

[168] H. Wymeersch, M. Z. Win, and J. Lien, "Cooperative localization for wireless networks," U.S. Patent 9,329,257, May 3, 2016.

[169] W. W.-L. Li, R. A. Iltis, and M. Z. Win, "A smartphone localization algorithm using RSSI and inertial sensor measurement fusion," in Proc. IEEE Global Telecomm. Conf., Atlanta, GA, Dec. 2013, pp. $3357-$ 3362

[170] S. Mazuelas, F. A. Lago, D. Gonzalez, A. Bahillo, J. Blas, P. Fernandez, R. Lorenzo, and E. Abril, "Dynamic estimation of optimum path loss model in a RSS possitioning system," in IEEE/ION Position, Location and Navigation Symposium, May 2008, pp. 679-684.

[171] M. R. Gholami, R. M. Vaghefi, and E. G. Ström, "RSS-based sensor localization in the presence of unknown channel parameters," IEEE Trans. Signal Process., vol. 61, no. 15, pp. 3752-3759, Aug. 2013.

[172] S. Yan, G. W. Peters, I. Nevat, and R. Malaney, "Location verification systems based on received signal strength with unknown transmit power," IEEE Commun. Lett., vol. 22, no. 3, pp. 650-653, Mar. 2018.

[173] B. Zhou, Q. Chen, and P. Xiao, "The error propagation analysis of the received signal strength-based simultaneous localization and tracking in wireless sensor networks," IEEE Trans. Inf. Theory, vol. 63, no. 6, pp. 3983-4007, Jun. 2017.

[174] B. Alavi and K. Pahlavan, "Modeling of the TOA-based distance measurement error using UWB indoor radio measurements," IEEE Commun. Lett., vol. 10, no. 4, pp. 275-277, Apr. 2006.

[175] X. Li and K. Pahlavan, "Super-resolution TOA estimation with diversity for indoor geolocation," IEEE Trans. Wireless Commun., vol. 3, no. 1, pp. 224-234, Jan. 2004

[176] I. Guvenc, Z. Sahinoglu, and P. V. Orlik, "TOA estimation for IR-UWB systems with different transceiver types," IEEE Trans. Microw. Theory Techn., vol. 54, no. 4, pp. 1876-1886, Jun. 2006.

[177] L. Stoica, A. Rabbachin, and I. Oppermann, "A low-complexity noncoherent IR-UWB transceiver architecture with TOA estimation," IEEE Trans. Microw. Theory Techn., vol. 54, no. 4, pp. 1637-1646, Jun. 2006.

[178] K. Yu and I. Oppermann, "Performance of UWB position estimation based on time-of-arrival measurements," in Proc. Int. Workshop on Ultra Wideband Syst., Oulu, Finland, May 2004, pp. 400-404.

[179] Y. Wang and K. C. Ho, "TDOA source localization in the presence of synchronization clock bias and sensor position errors," IEEE Trans. Signal Process., vol. 61, no. 18, pp. 4532-4544, Sep. 2013.

[180] L. Yang and K. C. Ho, "An approximately efficient TDOA localization algorithm in closed-form for locating multiple disjoint sources with 
erroneous sensor positions," IEEE Trans. Signal Process., vol. 57, no. 12, pp. 4598-4615, Dec. 2009.

[181] L. Cong and W. Zhuang, "Hybrid TDOA/AOA mobile user location for wideband CDMA cellular systems," IEEE Trans. Wireless Commun., vol. 1, no. 3, pp. 439-447, 2002.

[182] L. Xiong, "A selective model to suppress NLOS signals in angle-ofarrival (AOA) location estimation," in Proc. 9th IEEE Int. Symp. on Personal, Indoor and Mobile Radio Communications, vol. 1, Boston, MA, 1998, pp. 461-465.

[183] H.-J. Shao, X.-P. Zhang, and Z. Wang, "Efficient closed-form algorithms for AOA based self-localization of sensor nodes using auxiliary variables," IEEE Trans. Signal Process., vol. 62, no. 10, pp. 2580-2594, May 2014.

[184] M. Hawes, L. Mihaylova, F. Septier, and S. Godsill, "Bayesian compressive sensing approaches for direction of arrival estimation with mutual coupling effects," IEEE Trans. Aerosp. Electron. Syst., vol. 65, no. 3, pp. 1357-1368, Mar. 2017.

[185] I. Shames, A. N. Bishop, M. Smith, and B. D. Anderson, "Doppler shift target localization," IEEE Trans. Aerosp. Electron. Syst., vol. 49, no. 1, pp. 266-276, Jan. 2013

[186] J. How, N. Pohlman, and C. Park, "GPS estimation algorithms for precise velocity, slip and race-track position measurements," $S A E$ International, 2002.

[187] S. Chen and K. C. Ho, "Accurate localization of a rigid body using multiple sensors and landmarks," IEEE Trans. Signal Process., vol. 63, no. 24, pp. 6459-6472, Dec. 2015.

[188] K. Makaratat, T. W. C. Brown, S. Stavrou, and B. Evans, "Complex correlation on linear array angle-of-arrival estimation measurement of ultra wideband propagation channels," IET Microw. Antennas Propag., vol. 4, no. 9, pp. 1354-1369, Sep. 2010.

[189] X. Yuan, "Estimating the DOA and the polarization of a polynomialphase signal using a single polarized vector-sensor," IEEE Trans. Signal Process., vol. 60, no. 3, pp. 1270-1282, Mar. 2012.

[190] L. Liu and H. Liu, "Joint estimation of DOA and TDOA of multiple reflections in mobile communications," IEEE Access, vol. 4, pp. 38153823, Jun. 2016.

[191] J. A. Zhang, W. Ni, P. Cheng, and Y. Lu, "Angle-of-arrival estimation using different phase shifts across subarrays in localized hybrid arrays," IEEE Commun. Lett., vol. 20, no. 11, pp. 2205-2208, Nov. 2016.

[192] X. Li, "RSS-based location estimation with unknown pathloss model," IEEE Trans. Wireless Commun., vol. 5, no. 12, pp. 3626-3633, Dec. 2006.

[193] J. Li and R. Wu, "An efficient algorithm for time delay estimation," IEEE Trans. Signal Process., vol. 46, no. 8, pp. 2231-2235, Aug. 1998.

[194] T. Manickam, R. Vaccaro, and D. Tufts, "A least-squares algorithm for multipath time-delay estimation," IEEE Trans. Signal Process., vol. 42, no. 11, pp. 3229-3233, 1994.

[195] H. Saarnisaari, "ML time delay estimation in a multipath channel," in IEEE Proc. ISSTA 1996, 1996, pp. 1007-1011.

[196] N. C. Beaulieu, "Closed-form approximate to PDF of time-average power of bandlimited Gaussian process," IEE Electronics Letters, vol. 32, no. 21, pp. 1948-1950, Oct. 1996.

[197] V. Kostylev, "Energy detection of a signal with random amplitude," in Proc. IEEE Int. Conf. Commun., New York, USA, Apr. 2002, pp. 1606-1610.

[198] J. Salt and H. Nguyen, "Performance prediction for energy detection of unknown signals," IEEE Trans. Veh. Technol., vol. 57, no. 6, pp. 3900-3904, Nov. 2008.

[199] O. Olabiyi and A. Annamalai, "Further results on energy detection of random signals in gaussian noise," in Proc. IEEE Int. Conf. Connected Veh. and Expo, Las Vegas, USA, Dec. 2013, pp. 14-19.

[200] F. Digham, M.-S. Alouini, and M. K. Simon, "On the energy detection of unknown signals over fading channels," IEEE Trans. Commun., vol. 55, no. 1, pp. 21-24, Jan. 2007.

[201] A. Anttonen, A. Mammela, and A. Kotelba, "Error probability of energy detected multilevel PAM signals in lognormal multipath fading channels," in Proc. IEEE Int. Conf. Commun., Dresden, Germany, Jun. 2009 , pp. $1-5$

[202] S. Atapattu, C. Tellambura, and H. Jiang, "Performance of an energy detector over channels with both multipath fading and shadowing," IEEE Trans. Wireless Commun., vol. 9, no. 12, pp. 3662-3670, Dec. 2010

[203] A. Russ and M. K. Varanasi, "Noncoherent multiuser detection for nonlinear modulation over the Rayleigh-fading channel," IEEE Trans. Inf. Theory, vol. 44, no. 1, pp. 295-307, Jan. 2001.
[204] A. Rabbachin, T. Q. Quek, P. C. Pinto, I. Oppermann, and M. Z. Win, "Non-coherent UWB communication in the presence of multiple narrowband interferers," IEEE Trans. Wireless Commun., vol. 9, no. 11, pp. 3365-3379, Nov. 2010.

[205] E. D. Banta, "Energy detection of unknown deterministic signals in the presence of jamming," IEEE Trans. Aerosp. Electron. Syst., vol. 14, no. 2, pp. 384-386, Mar. 1978.

[206] E. Fishler, A. M. Haimovich, R. S. Blum, D. Chizhik, J. Leonard J. Cimini, and R. Valenzuela, "MIMO radar: an idea whose time has come," in Proc. of the IEEE Radar Conference, Apr. 2004, pp. 71-78.

[207] G. Zheng, B. Chen, and M. Yang, "Unitary ESPRIT algorithm for bistatic MIMO radar," Electronics Lett., vol. 48, no. 3, pp. 179-181, Feb. 2012.

[208] J. Li, D. Jiang, and X. Zhang, "DOA estimation based on combined unitary ESPRIT for coprime MIMO radar," IEEE Commun. Lett., vol. 21, no. 1, pp. 96-99, Jan. 2017.

[209] X. Zhang, L. Xu, L. Xu, and D. Xu, "Direction of departure (DOD) and direction of arrival (DOA) estimation in MIMO radar with reduceddimension MUSIC," IEEE Commun. Lett., vol. 14, no. 12, pp. 11611163, Dec. 2010.

[210] A. F. Molisch, D. Cassioli, C.-C. Chong, S. Emami, A. Fort, B. Kannan, J. Karedal, J. Kunisch, H. Schantz, K. Siwiak, and M. Z. Win, "A comprehensive standardized model for ultrawideband propagation channels," IEEE Trans. Antennas Propag., vol. 54, no. 11, pp. 31513166, Nov. 2006, special issue on Wireless Communications.

[211] J. O. Berger, J. M. Bernardo, and D. Sun, "The formal definition of reference priors," The Annals of Statistics, vol. 37, no. 2, pp. 905-938, 2009.

[212] R. E. Kalman, "A new approach to linear filtering and prediction problems," Trans. ASME J. Basic Eng., vol. 82, pp. 34-45, 1960.

[213] R. E. Kalman, P. L. Falb, and M. A. Arbib, Topics in Mathematical System Theory. New York: McGraw-Hill, 1969

[214] T. Kailath, "A view of three decades of linear filtering theory," IEEE Trans. Inf. Theory, vol. 20, no. 2, pp. 146-181, Mar. 1974.

[215] T. Kailath, A. H. Sayed, and B. Hassibi, Linear Estimation, ser. Prentice-Hall Information and System Sciences Series. Upper Saddle River, New Jersey 07458: Prentice Hall, 2000.

[216] S. K. Mitter, "Filtering and stochastic control: a historical perspective," IEEE Control Systems, vol. 16, no. 3, pp. 67-76, Jun. 1996.

[217] B. Ristic, M. S. Arulampalam, and N. Gordon, Beyond the Kalman Filter: Particle Filters for Tracking Applications. Norwood, MA: Artech House, 2004

[218] S. J. Julier and J. K. Uhlmann, "Unscented filtering and nonlinear estimation," Proc. IEEE, vol. 92, no. 3, pp. 401-422, Mar. 2004.

[219] S. Mazuelas, Y. Shen, and M. Z. Win, "Information coupling in cooperative localization," IEEE Commun. Lett., vol. 15, no. 7, pp. 737739, Jul. 2011

[220] S. Mazuelas, Y. Shen, and M. Z. Win, "Spatiotemporal information coupling in network navigation," IEEE Trans. Inf. Theory, vol. 64 no. 12 , pp. $7759-7779$, Dec. 2018.

[221] H. L. Van Trees, Detection, Estimation and Modulation Theory, Part 1. New York, NY: Wiley, 1968.

[222] J. Klemelä, Smoothing of Multivariate Data: Density Estimation and Visualization. John Wiley \& Sons, 2009.

[223] V. N. Vapnik, Statistical learning theory. New York: Wiley, 1998.

[224] D. Scott, Multivariate density estimation: theory, practice, and visualization. Wiley Com, 2009.

[225] S. Bartoletti, W. Dai, A. Conti, and M. Z. Win, "Wideband localization via range likelihood based on reduced dataset," in Proc. IEEE Canadian Workshop on Inf. Theory, St. John's, NL, Canada, Jul. 2015, pp. 93-96.

[226] A. H. Welsh, Aspects of Statistical Inference. Hoboken, NJ: John Wiley \& Sons, Inc., 2011 


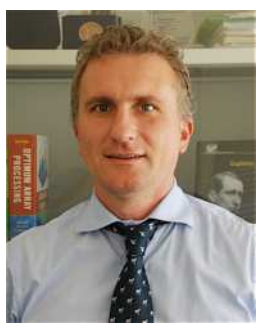

Andrea Conti (S'99-M'01-SM'11) received the Laurea (summa cum laude) in telecommunications engineering and the Ph.D. in electronic engineering and computer science from the University of Bologna, Italy, in 1997 and 2001, respectively.

$\mathrm{He}$ is a Professor at the University of Ferrara, Italy. Prior to joining the University of Ferrara, he was with the Consorzio Nazionale Interuniversitario per le Telecomunicazioni and with the IEIITConsiglio Nazionale delle Ricerche. In Summer 2001, he was with the Wireless Systems Research Department at AT\&T Research Laboratories. Since 2003, he has been a frequent visitor to the Wireless Information and Network Sciences Laboratory at the Massachusetts Institute of Technology, where he presently holds the Research Affiliate appointment. His research interests involve theory and experimentation of wireless systems and networks including network localization, distributed sensing, adaptive diversity communications, and network secrecy. He is recipient of the HTE Puskás Tivadar Medal and co-recipient of the IEEE Communications Society's Stephen O. Rice Prize in the field of Communications Theory and of the IEEE Communications Society's Fred W. Ellersick Prize.

Dr. Conti has served as editor for IEEE journals, as well as chaired international conferences. He has been elected Chair of the IEEE Communications Society's Radio Communications Technical Committee. He is a co-founder and elected Secretary of the IEEE Quantum Communications \& Information Technology Emerging Technical Subcommittee. He is an elected Fellow of the IET and has been selected as an IEEE Distinguished Lecturer.

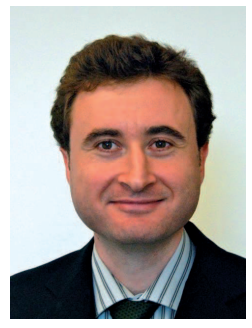

Santiago Mazuelas (M'10-SM'17) received the $\mathrm{Ph} . \mathrm{D}$. in Mathematics and Ph.D in Telecommunications Engineering from the University of Valladolid, Spain, in 2009 and 2011, respectively.

Since 2017 he has been Ramon y Cajal Researcher at the Basque Center for Applied Mathematics (BCAM). Prior to joining BCAM, he was a Staff Engineer at Qualcomm Corporate Research and Development from 2014 to 2017. He previously worked from 2009 to 2014 as Postdoctoral Fellow and Associate in the Laboratory for Information and Decision Systems (LIDS) at the Massachusetts Institute of Technology (MIT). His general research interest is the application of mathematics to solve engineering problems. Currently his work is primarily focused on statistical signal processing, machine learning, and data science.

Dr. Mazuelas is an Associate Editor for the IEEE Communications Letters and served as Co-chair for the Symposiums on Wireless Communications at the 2014 IEEE Globecom and at the 2015 IEEE ICC. He has received the Best Student Prize in Telecommunications Technical Engineering from University of Valladolid in 2006, the Best Doctorate Thesis Award from University of Valladolid in 2011, and the Young Scientist Prize from the Union RadioScientifique Internationale (URSI) Symposium in 2007. His papers received the IEEE Communications Society Fred W. Ellersick Prize in 2012, and Best Paper Awards from the IEEE ICC in 2013, the IEEE ICUWB in 2011, and the IEEE Globecom in 2011.

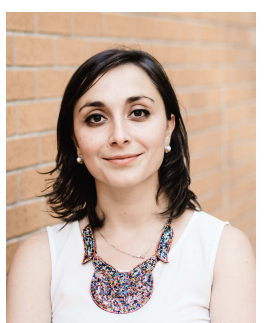

Stefania Bartoletti (S'12-M'16) received the Laurea degree (summa cum laude) in electronics and telecommunications engineering and the Ph.D. degree in information engineering from the University of Ferrara, Italy, in 2011 and 2015, respectively.

Since June 2016, she is recipient of a Marie Skłodowska-Curie Global Fellowship within the H2020 European Framework for a research project with the Massachusetts Institute of Technology and the University of Ferrara. Her research interests include theory and experimentation of wireless networks for passive localization and physical behavior analysis. She is recipient of the 2016 Paul Baran Young Scholar Award of the Marconi Society.

Dr. Bartoletti served as a Chair of the TPC for the IEEE ICC Workshop on Advances in Network Localization and Navigation (ANLN) from 2017 to 2019, and as reviewer for numerous IEEE journals and international conferences.

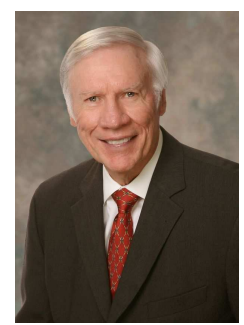

William C. Lindsey (S'61-M'63-SM'73-F'74LF'YY) received his Ph.D. degree in Electrical Engineering from Purdue University in 1962. He was the Supervisor of the Communications Research Group at Caltech's Jet Propulsion Laboratory (1962-1968), and the Founder, CEO, and President of LinCom Corporation (1972-2000). He joined the University of Southern California in 1968.

$\mathrm{He}$ is an internationally known expert with over 50 years of experience in the field of Communication Sciences. He authored three landmark systems engineering books on the subject of modulation-coding, demodulation-decoding, data detection, synchronization, and tracking for communication technology applications. His patents have been implemented in numerous communication satellite systems and ground station and mobile communications receivers located around the world.

Dr. Lindsey's synchronization and communications research has spearheaded the digital communications revolution and created novel technologies for space and mobile wireless communications. Lindsey's digital-data transition-tracking loop (DTTL) enabled the decoding of the first photos of Mars from NASA's Mariner spacecraft as well as images from the Voyager missions, and it was critical to space shuttle and space station communications efforts. It also led to the concept of "digital phase-locked systems on a chip" used in modern mobile radios and localization systems. He was one of the pioneering founders of the IEEE Communication Society. He was honored with the IEEE Third Millennium Medal in 2000 and the prestigious IEEE Eric E. Sumner Award in 2017. Dr. Lindsey is a member of the National Academy of Engineering and a Lifetime Fellow of the IEEE.

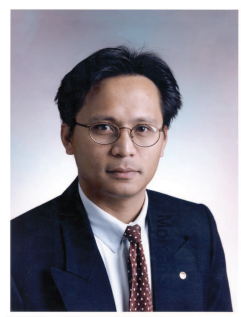

Moe Z. Win (''85-M'87-SM'97-F'04) is a Professor at the Massachusetts Institute of Technology (MIT) and the founding director of the Wireless Information and Network Sciences Laboratory. Prior to joining MIT, he was with AT\&T Research Laboratories and NASA Jet Propulsion Laboratory.

His research encompasses fundamental theories, algorithm design, and network experimentation for a broad range of real-world problems. His current research topics include network localization and navigation, network interference exploitation, and quantum information science. He has served the IEEE Communications Society as an elected Member-at-Large on the Board of Governors, as elected Chair of the Radio Communications Committee, and as an IEEE Distinguished Lecturer. Over the last two decades, he held various editorial positions for IEEE journals and organized numerous international conferences. Currently, he is serving on the SIAM Diversity Advisory Committee.

Dr. Win is an elected Fellow of the AAAS, the IEEE, and the IET. He was honored with two IEEE Technical Field Awards: the IEEE Kiyo Tomiyasu Award (2011) and the IEEE Eric E. Sumner Award (2006, jointly with R. A. Scholtz). Together with students and colleagues, his papers have received numerous awards. Other recognitions include the IEEE Communications Society Edwin H. Armstrong Achievement Award (2016), the International Prize for Communications Cristoforo Colombo (2013), the Copernicus Fellowship (2011) and the Laurea Honoris Causa (2008) from the Università degli Studi di Ferrara, and the U.S. Presidential Early Career Award for Scientists and Engineers (2004). He is an ISI Highly Cited Researcher. 